\title{
Checklist with first records for the Echinoderms of northern Tunisia (central Mediterranean Sea)
}

\author{
Hayfa Chammem 1,3, Jamila Ben Souissi ${ }^{1,2}$, Angel Pérez-Ruzafa ${ }^{3}$ \\ ${ }^{1}$ University of Tunis El Manar, Faculty of Sciences of Tunis, 2092 El Manar II, Tunisia. \\ (HC) (Corresponding author) E-mail: hayfa.chammem@um.es. ORCID-iD: https://orcid.org/0000-0002-6814-5834 \\ ${ }^{2}$ University of Carthage, National Agronomic Institute of Tunisia (INAT), 1082 Tunis, Tunisia. \\ (JBS) E-mail: jbensouissi@yahoo.com. ORCID-iD: https://orcid.org/0000-0003-1761-4204 \\ ${ }^{3}$ University of Murcia, Faculty of Biology, Campus de Espinardo, 30100 Murcia, Spain. \\ (APR) E-mail: angelpr@um.es. ORCID-iD: https://orcid.org/0000-0003-4769-8912
}

\begin{abstract}
Summary: Tunisia occupies a strategic biogeographic position in the Mediterranean Sea and the Strait of Sicily is considered a biogeographical boundary that separates the eastern and western basins. Despite the importance of marine biodiversity in Tunisia, the few studies of Echinodermata fauna in this region data from long ago. In order to update and produce a validated checklist of the echinoderms that occur in northern Tunisia, a study of this phylum was carried out between 2012 and 2016. Forty-five species were inventoried and distributed into the five living Echinodermata classes (Crinoidea, Asteroidea, Ophiuroidea, Echinoidea and Holothuroidea). New occurrences of four species from Tunisian marine waters [Asterina pancerii (Gasco, 1876), Luidia atlantidea (Madsen, 1950), Ophiactis virens (Sars, 1859) and Leptopentacta tergestina (Sars, 1857)], are cited and discussed here for the first time.
\end{abstract}

Keywords: echinoderms; new occurrences; biodiversity; Tunisia; Mediterranean Sea.

Listado con primeros registros de los equinodermos del norte de Túnez (Mediterráneo central)

Resumen: Túnez ocupa un área biogeográfica estratégica en el Mediterráneo. El estrecho tunecino-siciliano es considerado una frontera biogeográfica que separa las cubetas oriental y occidental. Sin embargo, a pesar de su interés, los estudios sobre la fauna de equinodermos de Túnez son antiguos y escasos. Con el fin de elaborar el inventario de los equinodermos de la región septentrional del mar de Túnez, se realizó un estudio de este filum entre los años 2012 y 2016. Se han inventariado cuarenta y cinco especies pertenecientes a las cinco clases actuales de Echinodermata (Crinoidea, Asteroidea, Ophiuroidea, Echinoidea y Holothuroidea). Cuatro especies [Asterina pancerii (Gasco, 1876), Luidia atlantidea (Madsen, 1950), Ophiactis virens (Sars, 1859) y Leptopentacta tergestina (Sars, 1857)] se han recolectado por primera vez en estas aguas.

Palabras clave: equinodermos; nuevas citas; biodiversidad; Túnez; mar Mediterráneo.

Citation/Como citar este artículo: Chammem H., Ben Souissi J., Pérez-Ruzafa A. 2019. Checklist with first records for the Echinoderms of northern Tunisia (central Mediterranean Sea). Sci. Mar. 83(3): 277-288. https://doi.org/10.3989/ scimar.04899.19A

Editor: X. Turon.

Received: December 12, 2018. Accepted: June 3, 2019. Published: July 2, 2019.

Copyright: ( 92019 CSIC. This is an open-access article distributed under the terms of the Creative Commons Attribution 4.0 International (CC BY 4.0) License.

\section{INTRODUCTION}

Tunisia occupies a central position between the western and eastern Mediterranean Sea. It is the northernmost point of the African continent $\left(36^{\circ} 347^{\prime} \mathrm{N}\right.$, $9^{\circ} 129^{\prime} \mathrm{E}$ ). The Strait of Sicily, from Cape Bon (northeastern Tunisia) to Mazara dell Vallo (Sicily, southern Italy), has been considered a biogeographical barrier that separates the eastern and western Mediterranean basins (Bianchi and Morri 2000, Mejri et al. 2009). This separation is not only important from the point of view of connectivity between two basins with different hydrographical conditions, but also because of its geological history after the Messinian crises that isolated the two basins and a time lag in recolonization by Atlantic species (Zenetos 2010, Coll et al. 2010, Lipej et al. 2017). In fact, several genetic investigations on fish and macro-invertebrates in their different life 
stages, have demonstrated that the Strait of Sicily acts as a genetic boundary for African Mediterranean Sea species (Pérez-Losada et al. 2007, Zitari-Chatti et al. 2009, Deli et al. 2017). The colonization of new species in the Mediterranean Sea, first by the Lessepsian invasions after the opening of the Suez Canal and, more recently, through the Strait of Gibraltar as climate change becomes more evident (Zenetos 2010), makes Tunisia the point of convergence of the two processes, with a significant stretch of coastline on each side of this "boundary".

The phylum Echinodermata includes marine invertebrate species and is composed of five living classes: Crinoidea, Asteroidea, Ophiuroidea, Echinoidea and Holothuroidea. They cover a wide range of biological strategies, habitats and depths. Echinoderms are found from the shallow intertidal to the abyssal zone, where they play an important role in the ecological processes of marine ecosystems.

Lack of research on Echinodermata is a knowledge gap regarding Tunisian marine biodiversity. Only two studies, by Cherbonnier (1956) and Gautier-Mechaz (1958), have published checklists of Tunisian echinoderms. These checklists are old and need to be updated regarding aspects such as climate change, invasive species, diversity estimation and marine protected areas. This phylum is currently cited associated with the megabenthic invertebrate inventories of Le Danois (1925), Azouz (1973), Ben Othman (1973), El Lakhrach et al. (2012). Other authors have focused on one particular class, generally one of economic interest such as Echinoidea (Sellem et al. 2001) or Holothurioidea (Louiz et al. 2003).

In order to update the inventory of marine diversity of Echinodermata species in the Tunisian Sea, research was performed between 2012 and 2016. The acquired data were used to produce a validated checklist of the Echinodermata of northern Tunisia.

\section{MATERIALS AND METHODS}

\section{Study area}

Echinoderms were sampled at 93 sites in eight locations along the northern coasts of Tunisia (Supplementary material Table S1). The study area extends over 300 $\mathrm{km}$ of the Tunisian coastline, from the Algerian-Tunisian border $\left(37^{\circ} 01^{\prime} 06.0^{\prime \prime} \mathrm{N}, 8^{\circ} 44^{\prime} 04.5^{\prime \prime} \mathrm{E}\right)$ to the Cape Bon Peninsula $\left(36^{\circ} 26^{\prime} 53.1^{\prime \prime} \mathrm{N} \mathrm{10} 0^{\circ} 51^{\prime} 36.5^{\prime \prime} \mathrm{E}\right)$. (Fig. 1).

This area of the central Mediterranean Sea is constantly affected by incoming Atlantic marine currents (Lubet and Azouz 1969, Azouz 1973). It is characterized by a continental shelf with a small, irregular plateau and a steep slope (Azouz 1973). The heterogeneity of its bottom type, with hard and soft substrates, enriches the biodiversity of northern Tunisia (Azouz 1973, Ayari and Afli 2003).

\section{Data collection}

The Echinodermata inventory was carried out from March 2012 to July 2016. A variety of sampling strategies were adopted depending on the substrate type (rocky or soft bottom, depth) and respecting the benthic bionomics of the Mediterranean Sea (Table 1). Specimens were collected using a dredge for inshore shallow areas at depths of less than $50 \mathrm{~m}$ and a professional benthic fishing trawl for offshore waters where the depth exceeds $50 \mathrm{~m}$. Hand collection and diving were used for mid- and infralittoral levels $(<5 \mathrm{~m})$ (Supplementary material Table S2).

\section{Taxonomic work}

The collected material was measured, photographed and preserved in ethanol. Specimens were identified based on external morphology and internal

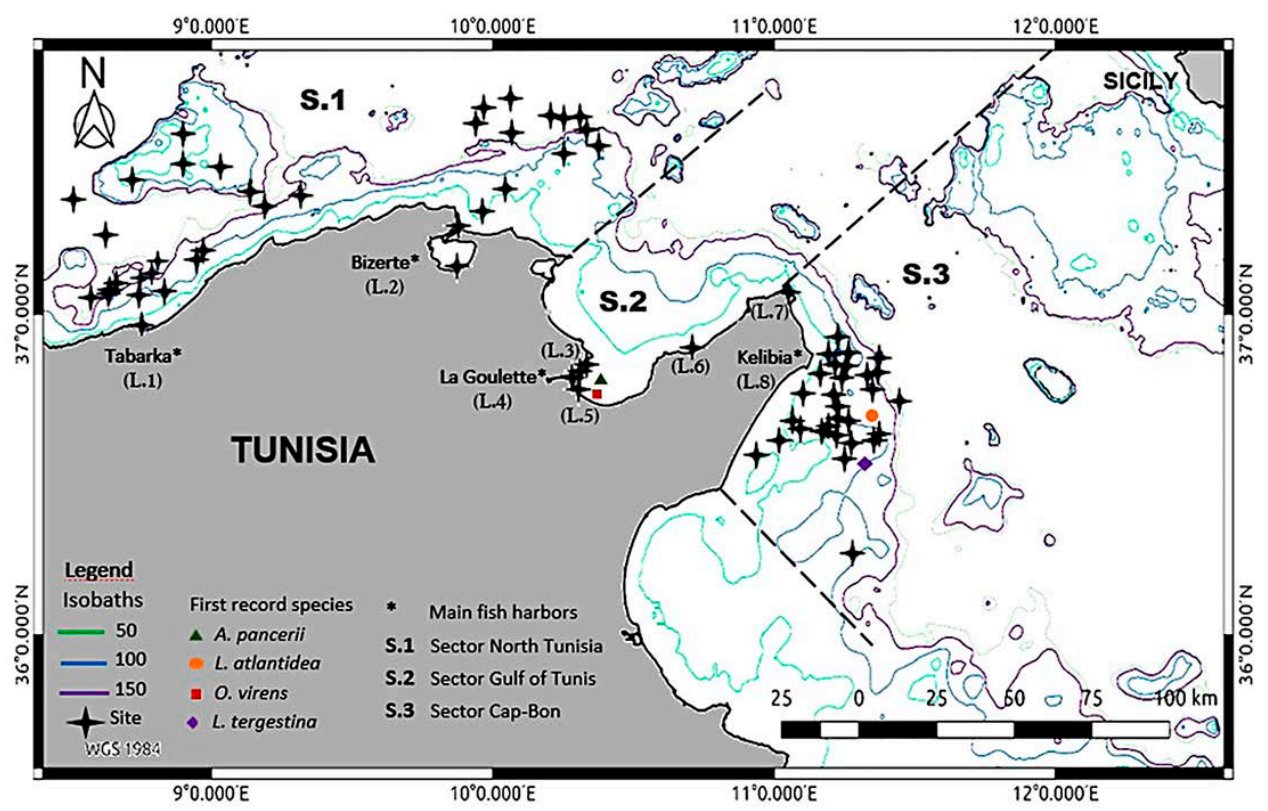

Fig. 1. - Map of the locations (L.) in northern Tunisian waters sampled for echinoderms, also showing the sites of new records: Asterina pancerii (green), Luidea atlantidea (orange), Ophiactis virens (red), Leptopentacta tergestina (purple). 
Table 1. - The checklist of Echinodermata of northern Tunisia. M, Mediterranean Sea; A, Atlantic Ocean; ME, Mediterranean endemics; C, cosmopolitan; R, Red Sea; A, Algae; Cy, Cymodocea; G, gorgonian; S, sandy bottom; M, muddy bottom; R, rocky bottom; *, first records of species; Abundance, total number of individuals; Location (L) from Figure 1.

\begin{tabular}{|c|c|c|c|c|c|}
\hline Taxa & Distribution & Depth range $(\mathrm{m})$ & Habitat & Abundance & Location $(\mathrm{L})$ \\
\hline \multicolumn{6}{|l|}{ Class CRINOIDEA } \\
\hline \multicolumn{6}{|l|}{ Family Antedonidae } \\
\hline Antedon bifida (Pennant, 1777) & $\mathrm{M}, \mathrm{A}$ & $50-190$ & $\mathrm{~S}, \mathrm{M}, \mathrm{R}$ & 50 & $1,2,8$ \\
\hline Antedon mediterranea (Lamarck, 1816) & ME & $50-190$ & $\mathrm{~S}, \mathrm{M}, \mathrm{R}$ & 68 & $1,2,8$ \\
\hline Leptometra phalangium (Müller, 1841) & $\mathrm{M}, \mathrm{A}$ & $72-194$ & $\mathrm{~S}, \mathrm{M}$ & 33 & 1,8 \\
\hline \multicolumn{6}{|l|}{ Class ASTREROIDEA } \\
\hline \multicolumn{6}{|l|}{ Family Asteriidae } \\
\hline Coscinasterias tenuispina (Lamarck, 1816) & $\mathrm{M}, \mathrm{A}$ & $20-51$ & $\mathrm{~S}$ & 2 & 2,8 \\
\hline Marthasterias glacialis (Linnaeus, 1758) & $\mathrm{M}, \mathrm{A}$ & $75-220$ & $\mathrm{~S}, \mathrm{M}, \mathrm{R}$ & 11 & 2,8 \\
\hline \multicolumn{6}{|l|}{ Family Asterinidae } \\
\hline Anseropoda placenta (Pennant, 1777) & $\mathrm{M}, \mathrm{A}$ & $185-220$ & $\mathrm{~S}$ & 1 & 8 \\
\hline Asterina gibbosa (Pennant, 1777) & $\mathrm{M}, \mathrm{A}$ & $0.45-0.65$ & $\mathrm{R}, \mathrm{A}$ & 13 & 3,5 \\
\hline Asterina pancerii (Gasco, 1876)* & ME & $3-5$ & $\mathrm{Cy}$ & 3 & 4 \\
\hline \multicolumn{6}{|l|}{ Family Astropectinidae } \\
\hline Astropecten aranciacus (Linnaeus, 1758) & $\mathrm{M}, \mathrm{A}$ & $51-177$ & $\mathrm{~S}, \mathrm{M}$ & 24 & 2,8 \\
\hline Astropecten bispinosus (Otto, 1823) & $\mathrm{M}, \mathrm{A}$ & $1-35$ & $\mathrm{~S}, \mathrm{M}$ & 11 & $2,3,4$ \\
\hline Astropecten irregularis (Pennant, 1777) & $\mathrm{M}, \mathrm{A}$ & $50-220$ & S & 33 & $1,2,8$ \\
\hline Astropecten jonstoni (Delle Chiaje, 1827) & ME & $3-5$ & $\mathrm{~S}$ & 1 & 4 \\
\hline Tethyaster subinermis (Philippi, 1837) & $\mathrm{M}, \mathrm{A}$ & $50-220$ & $\mathrm{~S}, \mathrm{M}$ & 39 & $1,2,8$ \\
\hline \multicolumn{6}{|l|}{ Family Chaetasteridae } \\
\hline Chaetaster longipes (Retzius, 1805) & $\mathrm{M}, \mathrm{A}$ & $70-170$ & $\mathrm{~S}, \mathrm{M}$ & 21 & $1,2,8$ \\
\hline Family Echinasteridae & & & & & \\
\hline Echinaster (Echinaster) sepositus (Retzius, 1783) & $\mathrm{M}, \mathrm{A}$ & $3-220$ & $\mathrm{~S}, \mathrm{M}, \mathrm{R}$ & 96 & $1,2,8$ \\
\hline Family Luidiidae & & & & & \\
\hline Luidia atlantidea Madsen, $1950 *$ & A & $65-95$ & $\mathrm{~S}$ & 1 & 8 \\
\hline Luidia sarsii sarsii Düben and Koren in Düben, 1844 & $\mathrm{M}, \mathrm{A}$ & $175-193$ & M & 1 & 1 \\
\hline Family Ophidiasteridae & & & & & \\
\hline Hacelia attenuata Gray, 1840 & $\mathrm{M}, \mathrm{A}$ & $70-85$ & $\mathrm{R}$ & 1 & 1 \\
\hline Class OPHIUROIDEA & & & & & \\
\hline Family Amphiuridae & & & & & \\
\hline Amphipholis squamata (Delle Chiaje, 1828) & $\mathrm{C}$ & $0.4-0.6$ & A & 16 & 5 \\
\hline Family Gorgonocephalidae & & & & & \\
\hline Astrospartus mediterraneus (Risso, 1826) & $\mathrm{M}, \mathrm{A}$ & $98-105$ & $\mathrm{~S}$ & 2 & 8 \\
\hline Family Ophiacanthidae & & & & & \\
\hline Ophiacantha setosa (Bruzelius, 1805) & $\mathrm{M}, \mathrm{A}$ & $70-165$ & $\mathrm{G}$ & 54 & 1 \\
\hline Family Ophiactidae & & & & & \\
\hline Ophiactis savignyi (Müller and Troschel, 1842) & $\mathrm{C}$ & $3-5$ & $\mathrm{~S}$ & 1 & 5 \\
\hline Ophiactis virens (M. Sars, 1859) * & $\mathrm{M}, \mathrm{A}$ & $0.4-0.6$ & $\mathrm{~A}$ & 184 & 5 \\
\hline Family Ophiocomidae & & & & & \\
\hline Ophiocomina nigra (Abildgaard in O.F. Müller, 1789) & $\mathrm{M}, \mathrm{A}$ & $50-58$ & M & 1 & 2 \\
\hline Family Ophiodermatidae & & & & & \\
\hline Ophioderma longicauda (Bruzelius, 1805) & $\mathrm{M}, \mathrm{A}$ & 0.65 & $\mathrm{R}, \mathrm{A}$ & 1 & 7 \\
\hline Family Ophiomyxidae & & & & & \\
\hline Ophiomyxa pentagona (Lamarck, 1816) & $\mathrm{M}, \mathrm{A}$ & $50-210$ & $\mathrm{~S}, \mathrm{M}, \mathrm{R}$ & 81 & 2,8 \\
\hline Family Ophiotrichidae & & & & & \\
\hline Ophiothrix quinquemaculata (Delle Chiaje, 1828) & ME & $72-175$ & $\mathrm{~S}, \mathrm{M}, \mathrm{R}$ & 21 & 2 \\
\hline Family Ophiuridae & & & & & \\
\hline Ophiura ophiura (Linnaeus, 1758) & $\mathrm{M}, \mathrm{A}$ & $3-194$ & $\mathrm{~S}, \mathrm{M}, \mathrm{R}$ & 124 & $1,2,4,8$ \\
\hline Class ECHINOIDEA & & & & & \\
\hline Family Arbaciidae & & & & & \\
\hline Arbacia lixula (Linnaeus, 1758) & $\mathrm{M}, \mathrm{A}$ & $0.25-5$ & $\mathrm{~S}, \mathrm{R}$ & 12 & $1,2,7$ \\
\hline Family Cidaroidae & & & & & \\
\hline Cidaris cidaris (Linnaeus, 1758) & $\mathrm{M}, \mathrm{A}$ & $50-220$ & $\mathrm{~S}, \mathrm{M}, \mathrm{R}$ & 114 & $1,2,8$ \\
\hline Stylocidaris affinis (Mortensen, 1909) & $\mathrm{C}$ & $50-220$ & $\mathrm{~S}, \mathrm{M}, \mathrm{R}$ & 114 & $1,2,8$ \\
\hline Family Diadematidae & & & & & \\
\hline Centrostephanus longispinus (Philippi, 1845) & $\mathrm{M}, \mathrm{A}$ & $50-220$ & $\mathrm{~S}, \mathrm{M}$ & 74 & 2,8 \\
\hline Family Echinidae & & & & & \\
\hline Gracilechinus acutus Lamarck, 1816 & $\mathrm{M}, \mathrm{A}$ & $50-125$ & $\mathrm{~S}, \mathrm{R}$ & 18 & 8 \\
\hline Family Parechinidae & & & & & \\
\hline Paracentrotus lividus (Lamarck, 1816) & $\mathrm{M}, \mathrm{A}$ & $0.2-6$ & $\mathrm{~S}, \mathrm{R}$ & 48 & $1,2,7$ \\
\hline Family Spatangidae & & & & & \\
\hline Spatangus purpureus (O.F. Müller, 1776) & $\mathrm{M}, \mathrm{A}$ & $3-5$ & $\mathrm{~S}$ & 1 & 8 \\
\hline Family Toxopneustidae & & & & & \\
\hline Sphaerechinus granularis (Lamarck, 1816) & $\mathrm{M}, \mathrm{A}$ & $0.6-5$ & $\mathrm{R}, \mathrm{A}$ & 2 & 7 \\
\hline Class HOLOTHUROIDEA & & & & & \\
\hline Family Cucumariidae & & & & & \\
\hline Hemiocnus syracusanus (Grube, 1840) & M & $3-5$ & $\mathrm{~S}$ & 1 & 4 \\
\hline Leptopentacta elongata (Düben and Koren, 1846) & $\mathrm{M}, \mathrm{A}$ & $77-145$ & $\mathrm{~S}$ & 1 & 8 \\
\hline Leptopentacta tergestina (M. Sars, 1857) * & ME & $77-145$ & $\mathrm{~S}$ & 3 & 8 \\
\hline Family Holothuriidae & & & & & \\
\hline Holothuria (Holothuria) mammata Grube, 1840 & ME & $3-8$ & $\mathrm{~S}, \mathrm{R}, \mathrm{A}$ & & 2 \\
\hline Holothuria (Holothuria) tubulosa Gmelin, 1791 & $\mathrm{M}, \mathrm{A}, \mathrm{R}$ & $0.2-185$ & $\mathrm{~S}, \mathrm{M}, \mathrm{R}, \mathrm{A}$ & 40 & $1,2,7$ \\
\hline Holothuria (Platyperona) sanctori Delle Chiage, 1823 & $\mathrm{M}, \mathrm{A}, \mathrm{R}$ & $0.2-0.4$ & $\mathrm{R}, \mathrm{A}$ & 8 & 7 \\
\hline Holothuria (Roweothuria) poli Delle Chiaje, 1824 & $\mathrm{M}, \mathrm{A}, \mathrm{R}$ & $0.2-8$ & $\mathrm{~S}, \mathrm{R}, \mathrm{A}$ & 16 & 2,7 \\
\hline Holothuria (Thymiosycia) impatiens (Forsskål, 1775) & $\mathrm{C}$ & 0.45 & $\mathrm{R}$ & 1 & 6 \\
\hline Family Stichopodidae & & & & & \\
\hline Parastichopus regalis (Cuvier, 1817) & $\mathrm{M}, \mathrm{A}$ & $0.2-194$ & $\mathrm{~S}, \mathrm{M}, \mathrm{R}$ & 58 & 1.8 \\
\hline
\end{tabular}


anatomy following the taxonomic criteria of Mortensen (1927), Tortonese (1965), Koehler (1969) and Clark and Downey (1992). The nomenclature followed the World Register of Marine Species (WoRMS Editorial Board 2019). Sometimes, morphological characters can be ambiguous. For example, spicules of some of our individuals of sea cucumber from the genus Holothuria showed confusing anatomical and morphological characteristics. In fact, buttons of our individuals of Holothuria [Holothuria (Holothuria) tubulosa (Gmelin, 1791) and Holothuria (Roweothuria) poli (Delle Chiaje, 1824)] were twisted, which is a typical button characteristics of the eastern Atlantic species Holothuria (Vaneyothuria) lentiginosa (Marenzeller von, 1892) (Miller and Pawson 1979). The same species was cited in the Alboran Sea by Pérez-Ruzafa and López-Ibor (1988). In these cases, for the determination and identification of individuals, morphological studies were completed with genetic analyses.

\section{Molecular analysis}

Samples. To clarify the taxonomic status of these individuals, a genetic analysis was performed on 28 samples from the genus Holothuria, including the 17 doubtful specimens from northern Tunisia, in addition to 10 specimens of $H$. poli and $H$. tubulosa from Spain and a single specimen of $H$. lentiginosa from the Canary Islands from the collection of Dr Angel PérezRuzafa at the Department of Ecology and Hydrology of the Faculty of Biology (University of Murcia). We used as an outgroup taxon 6 individuals of $H$. (Panningothuria) forskali Delle Chiaje, 1823 and $H$. (Platyperona) sanctori Delle Chiaje, 1823 . The sequences were taken from Genebank (GenBank accession numbers GQ214761-GQ214762, EU220819, KY774322, GQ214763-GQ214764).

DNA extraction, PCR amplification and sequencing. DNA was extracted from 15-25 mg of muscle tissue of holothurian samples, which was conserved in ethanol following the standard protocol of Sambrook et al. (1989). Only the mitochondrial gene subunit I of cytochrome oxidase (COI) (ca. $650 \mathrm{bp}$ ) was amplified. The primers used for the amplification were CO1eI 5'ATAATGATA GGAGGRTTTGG 3' and CO1eII 5'GCTCGTGTRTCTACRTCCAT 3' (Palumbi 1996, Borrero-Pérez et al. 2009). Amplifications were carried out in a12 $\mu \mathrm{L}$ final volume of reaction mixture containing $1.2 \mu \mathrm{L}$ of $10 \times$ buffer (Biotools), $0.6 \mu \mathrm{L}$ $\mathrm{MgCl} 2(50 \mathrm{Mm}), 0.24 \mu \mathrm{L}$ dNTP $(10 \mathrm{mM}), 0.6 \mu \mathrm{L}$ of each primer $(10 \mu \mathrm{M}), 0.6 \mu \mathrm{L}$ BSA $(20 \mathrm{mg} / \mathrm{ml}), 0.1 \mu \mathrm{L}$ of Taq DNA polymerase (5U/ $\mu \mathrm{L}$ ) (Biotools) and $1 \mu \mathrm{L}$ of genomic DNA (10 ng/uL). The complete PCR cycle was $94^{\circ} \mathrm{C}$ for 3 minutes, then 40 cycles of denaturation at $94^{\circ} \mathrm{C}$ for $30 \mathrm{~s}$, annealing at $50^{\circ} \mathrm{C}$ for $30 \mathrm{~s}$ and extension at $72^{\circ} \mathrm{C}$ for $20 \mathrm{~s}$, followed by a $20 \mathrm{~min}$ final extension time at $72^{\circ} \mathrm{C}$ (Uthicke et al. 2005). PCR products were visualized on $1 \%$ agarose gels. Purified DNA was sequenced at the Molecular Biology section of the Research Support Service at the University of Murcia (Spain) using Big Dye Terminator Cycle Sequencing v. ABI Prism 310 technology (Applied Biosystems).
Phylogenetic reconstruction. Twenty-eight sequences of $500 \mathrm{bp}$ were edited and aligned using ClustalW as a default alignment parameter of the MEGA program, version 7 (Kumar et al. 2016). The DNA sequences were analysed to conduct a neighbourjoining tree using MEGA version 7 (Kumar et al. 2016). Pairwise nucleotide distances were calculated using the Kimura 2-parameter (K2P) model of base substitution (Kimura 1980).

Samples of the collected martial are deposited in the zoology collection of the University of Murcia (UMCZ).

\section{RESULTS}

\section{Faunal diversity}

Forty-five echinoderm species were collected and identified in northern Tunisia waters (Table 1). They belonged to the five classes of Echinodermata and comprised three sea lilies (Crinoidea), 15 starfishes (Asteroidea), 10 brittle stars (Ophiuroidea), 8 sea urchins (Echinoidea) and 9 sea cucumbers (Holothuroidea). They were divided into 32 genera and 27 families.

All the inventoried species are present in the Mediterranean Sea, except for the starfish-Luidia atlantidea (Madsen, 1950), which is an Atlantic species recently recorded in the Alboran Sea (Gallardo-Roldán et al. 2015).

Six of the collected species are endemic in the Mediterranean Sea, namely: Asterina pancerii (Gasco, 1876), Astropecten jonstoni (Delle Chiaje, 1827), Holothuria (Holothuria) mammata (Grube, 1840), Leptopentacta tergestina (Sars, 1857), Ophiothrix quinquemaculata (Delle Chiaje, 1828) and Antedon mediterranea (Lamarck, 1816). Four others have a wide distribution and are cosmopolitan: Amphipholis squamata (Delle Chiaje, 1828), Ophiactis savignyi (Müller and Troschel, 1842), Holothuria (Thymiosycia) impatiens (Forsskål, 1775) and Stylocidaris affinis (Mortensen, 1909).

Four collected species were first records for $\mathrm{Tu}-$ nisia: Asterina pancerii (Gasco, 1876), Luidia atlantidea (Madsen, 1950), Ophiactis virens (Sars, 1859) and Leptopentacta tergestina (Sars, 1857). Two are exclusively Mediterranean species (Asterina pancerii and Leptopentacta tergestina), and one is an Atlantic species (Luidia atlantidea) (Fig. 2)

\section{New occurrences}

The new species recorded for the first time in the present work (Fig. 3, Table 1) are two asteroids (Asterina pancerii, Luidia atlantidea), one ophiuroid (Ophiactis virens) and one holothurian (Leptopentacta tergestina) belonging to three Echinodermata classes.

Class ASTEROIDEA Blainville, 1830 Order VALVATIDA Perrier, 1884

Family ASTERINIDAE Gray, 1840 Genus Asterina Nardo, 1834 

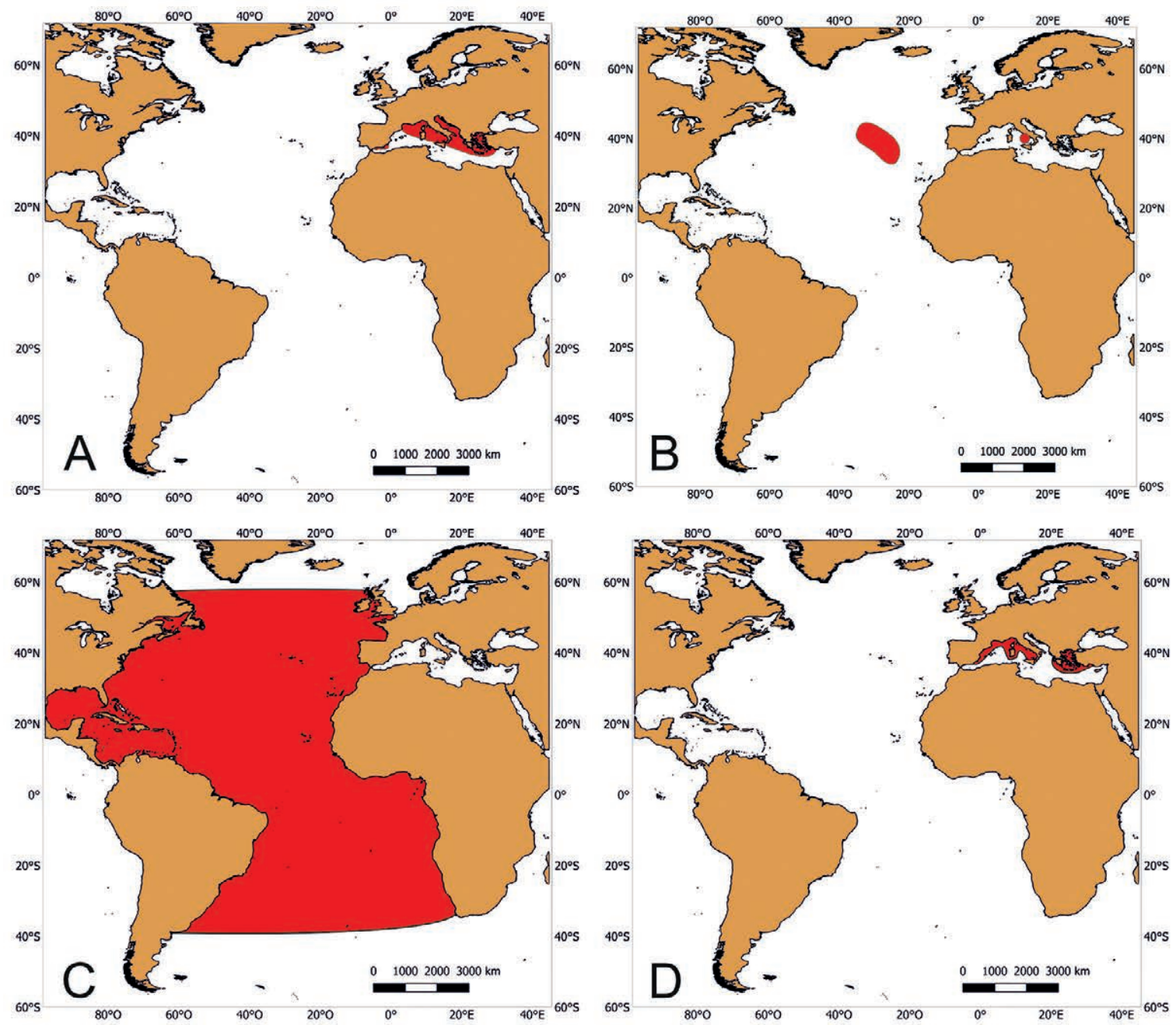

Fig. 2. - Maps of the geographic distribution (in black) of the species newly recorded in Tunisia: Leptopentacta tergestina (A), Ophiactis virens (B), Luidea atlantidea (C) and Asterina pancerii (D) (according to WoRMS, and López-Márquez et al. 2018, for Asterina pancerii).

Asterina pancerii (Gasco, 1876) Figs 2D, 3A-B

Asteriscus pancerii Gasco 1870: 86-90. Gasco 1876: 38-40 Asterina gibbosa var. panceri Khohler 1924: 133-134

Asterina pancerii Tortonese 1965: 172-173. Oliver et al. 1997: 103107. Tanti and Schembri 2006: 163-165.

Diagnosis. Flat body with a noticeable pentagonal shape; five rays, short and rounded with two or three papulae; abactinal plates close to each other and covered by spinelets; actinal gonopore are present; subambilacral and supactinal plates are absent; skeletal plates are few and large; actinal plates are distinct with three actinal spines per plate; numerous suboral spines with three usually tending to form a row parallel to the oral furrow spines.

Description. A very small starfish, it is pentagonal in shape and has several colours (brick red or purple, green, olive green or blue) (Tortonese 1965, Oliver et al. 1997). Its diameter does not exceed $15 \mathrm{~mm}$. It has a flat form, with no superambulacral and superactinal plates. It has three suboral spines and gonopores on the ventral side (Clark and Downey 1992).
Examined material. Three specimens. Sector and location: Gulf of Tunis (S.2/L.4). Depth: 3-5 m. Substrates: associated with seagrass Cymodocea nodosa (Ascherson, 1870) (Table 1).

Distribution. Mediterranean Sea. It has been reported in several Mediterranean regions: France (Tortonese 1965), Murcia (Galán et al. 1982) and Mallorca (Oliver et al. 1997) in Spain, Athens (Tortonese 1965), Turkey (Özaydın et al. 1995) and Tripoli (Tortonese 1965). The species has been recorded in several localities in Spain, including Ibiza and Mallorca (Ballesteros et al. 1987, Oliver et al. 1997), Almeria, Murcia and Alicante (Luque and Templado 2004, Moreno et al. 2008). Recently López-Márquez et al. (2018) provided molecular evidence that the morphological identification of the specimens of Asterina pancerii from Alicante is incorrect and corresponds to A. phylactica (Emson and Crump, 1979).

Remarks. Asterina pancerii is a very small asteroid. Its morphology, which is extremely similar to that of juvenile Asterina gibbosa (Pennant, 1777), has led some authors (Hattour and Ben Mustapha 2015) to re- 

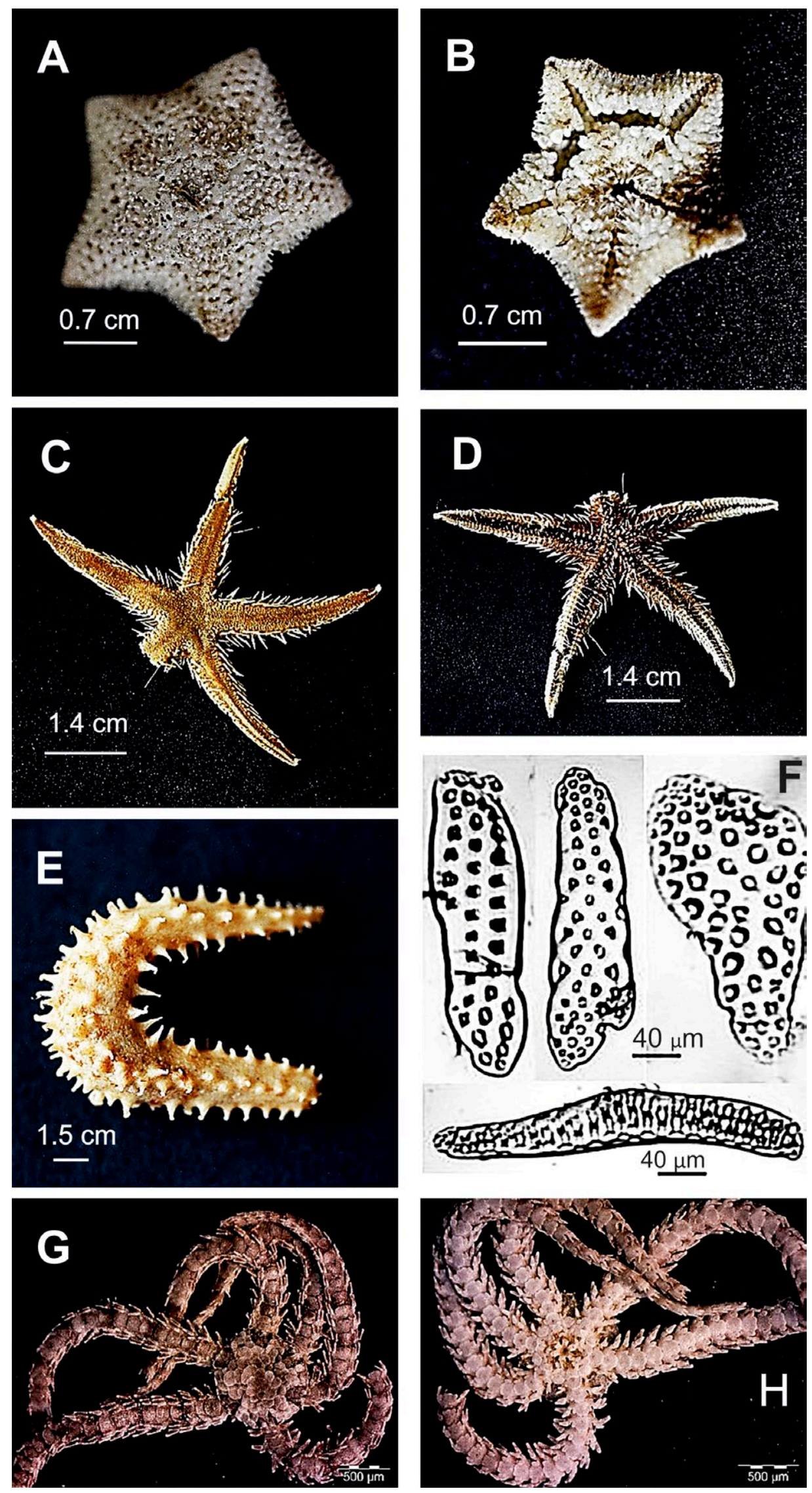

Fig. 3. - Asterina pancerii aboral view (A) and oral view (B); scale bar $1 \mathrm{~cm}$. Luidea atlantidea aboral view (C) and oral view (D); scale bar $1 \mathrm{~cm}$. Leptopentacta tergestina lateral view (E); scale bar $0.5 \mathrm{~cm}$. Body wall ossicules of Leptopentacta tergestina $(\mathrm{F}) ;$ scale bar $0.5 \mathrm{~cm}$. Ophiactis virens aboral view (J) and oral view $(\mathrm{H})$. 
port its presence in Tunisian waters (Gulf of Gabès). However, it was not cited in the final checklist of the same study.

Order PAXILLOSIDA Perrier, 1884

Family LUIDIIDAE Sladen, 1889

Genus Luidia Forbes, 1839

Luidia atlantidea Madsen, 1950

Fig. 2C, 3C-D

Luidia africana Doderlein 1920: 288-289 [Non L. africana Sladen, 1889]

Luidia atlantidea Madsen 1950: 192-198. Nataf and Cherbonnier 1973: 76-80. Clark and Downey 1992: 10-11.

Diagnosis. Flat body with five long thin arms; rays not very robust and narrow; abactinal paxillae with two marginal longitudinal series on each side with a white colour; coarser spinelets; the number of supermarginal paxillae is around 15 to 20, with rounded and flattened shape; lateral alignment of inferomarginal plates with two or three large and erect spines; marginal spines with dark base and white tips; presence of large pedicellaria on furrow face of each oral plate.

Description. It has five long, flattish arms with the presence of a marked main line of paxillae, arranged longitudinally (Clark and Downey 1992, GallardoRoldán et al. 2015). Central spinelets are distinctly coarser than peripheral ones. Supermarginal paxillae are rounded. Abactinal paxillae with two matching longitudinal lateral series on each side. Adambulacral plates with three large spines in a line at right-angles to the furrow. The central spinelets are distinctly coarser than the peripheral ones. Colour is grey with a white stripe along the supermarginal paxillae, white below, with dark purple marginal spines and white tips (Clark and Downey 1992). The diameter is about $6 \mathrm{~cm}$.

Examined material. One specimen. Sector and location: Cape Bon (S.3/L.8). Substrates: Sand. Depth: 65-95 m (Table 1).

Distribution. Atlantic Ocean. It is present along the Atlantic coast from Morocco to Zaire, including the Cape Verde Islands (Clark and Downey 1992, Entrambasaguas 2008).

Remarks. The genus Luidia is represented by two species in the Mediterranean Sea: L. sarsii sarsii (Düben and Koren in Düben, 1844) and L. ciliaris (Philippi, 1837) (Cherbonier 1956, Tortonese 1965, Koehler 1969). The main difference between these two Mediterranean species is the number of arms: more than five in L. ciliaris (Cherbonier 1956, Tortonese 1965, Koehler 1969). In addition, L. atlantidea differ from L. sarsii sarsii in the number of lateral paxillae (more than 17 for L. sarsii sarsii) and the central and peripheral spinelets, which are uniform (Clark and Downey 1992).

Class OPHIUROIDEA Gray, 1840 Order OPHIURIDA Müller and Troschel, 1840
Family OPHIACTIDAE Matsumoto, 1915

Genus Ophiactis Lütken, 1856

Ophiactis virens (M. Sars, 1859)

Figs 2B, 3G-H

Amphiura virens Sars 1859: 95.

Ophiactis virens Simroth 1876: 417-485. Koehler, 1924: 294. Tortonese 1965: 238-239.

Diagnosis. Small brittle star with six long, thin arms; small disc, rounded and convex, covered by small irregular plates; peripheral plates have a very short and conical spinelet; six triangular radial shields, very small, more or less sunken and distally joined; two mouth papillae; four radial spines; dorsal plates of arms very broad; no genital slits.

Description. It is a very small brittle star, with a disc diameter of 3-5 mm, characterized by the presence of six arms (Tortonese 1965, Koehler 1969). Disc colour is a yellowish-grey or is greenish with darker spots (Koehler 1924, 1969, Tortonese 1965). Dorsal disc is covered by plates with six triangular radials shields. Two mouth papillae on each side of jaw with four small arm spines. Dorsal plates of arms are very broad and without genital slits (Mortensen 1927, Koehler 1969).

Examined material. 184 individuals. Sector and location: Gulf of Tunis (S.2/L.5). Depth: 0.40-0.60 m. Substrates: Algae (Table 1).

Distribution. Atlantic Ocean and Mediterranean Sea. It has been recorded from the west coast of Africa to the archipelagos of Azores, Madeira, Cape Verde and the Gulf of Gascony (Marques 1980, Entrambasaguas 2008), Italy (Koehler 1924, Tortonese 1965), and Turkey (Özaydın et al. 1995, Öztoprak 2014).

Remarks. Ophitactis virens is morphologically close to Ophiactis savignyi (Müller and Troschel, 1842), which is a cosmopolitan species characterized by the absence or the presence of one or two papillae, oral shields with rounded edges and five thorny arm spines (Clark 1918).

Class HOLOTHUROIDEA Brin, 1860

Order DENDROCHIROTIDA Grube, 1840

Family CUCUMARIIDAE Ludwig, 1894

Genus Leptopentacta Clark, 1938

Leptopentacta tergestina (M. Sars, 1857) Figs 2A, 3E-F

Cucumaria incurvata Perrier 1886: 497

Cucumaria tergestina Sars 1859: 127. Koehler 1924: 158-160.

Trachythyone tergestina (M. Sars, 1857) Panning 1949: 426. Tortonese 1965: 83-85.

Diagnosis. Small species with a curved body; ambulacral feet are small, rigid, pointed and conical; they are arranged in two parallel rows; spicules are large and have an irregular shape; perforated plates which are large and irregular $(30-50 \mu \mathrm{m})$ with numerous perforations; irregular and curved rods. 


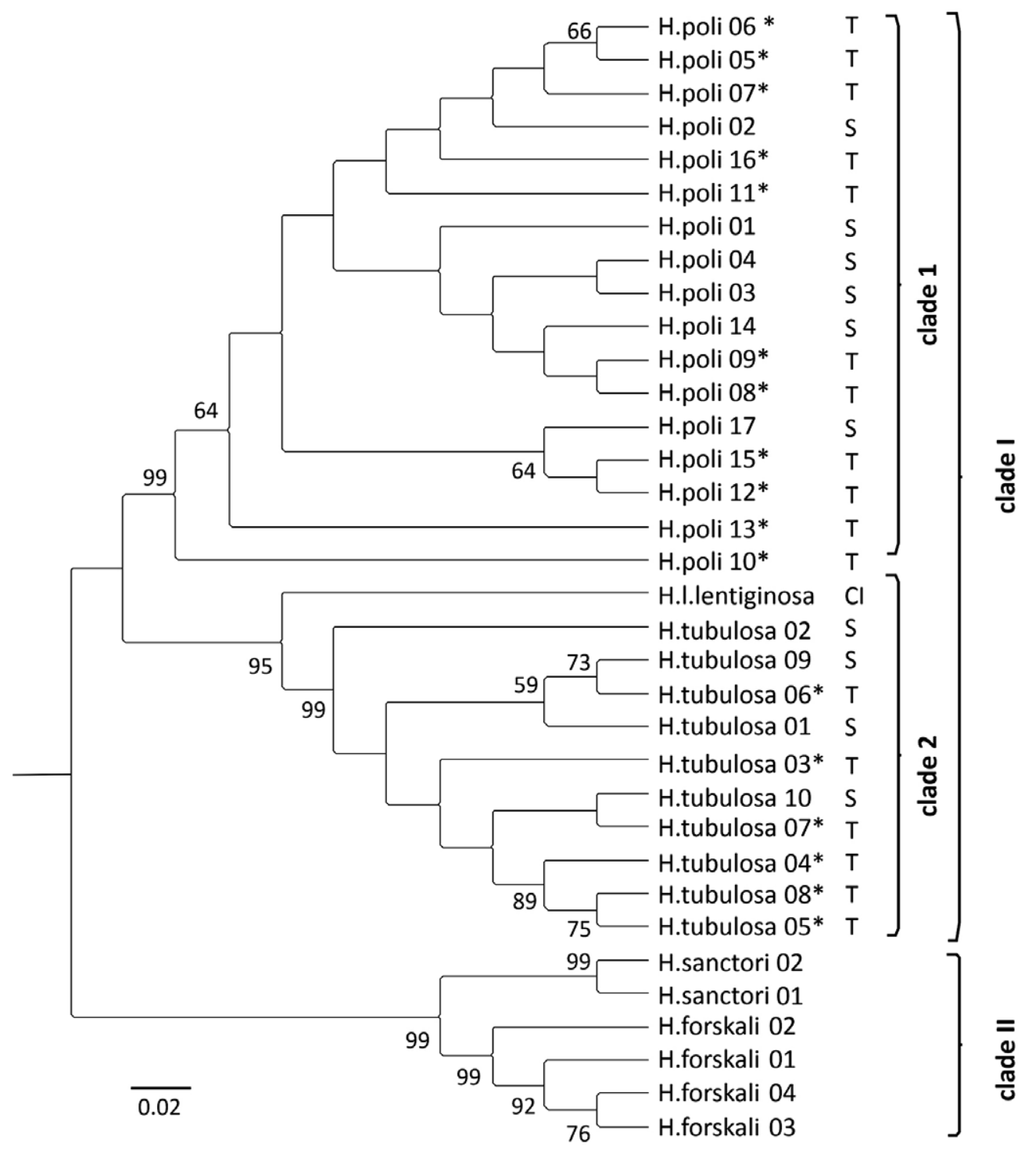

Fig. 4. - DNA sequence analysis of sea cucumbers from the genus Holothuria. Neighbour-joining tree analysis of 500 bp COI fragments based on p-distance. The bootstrap consensus tree inferred from 10000 replicates. Only bootstrap value branches exceeding $50 \%$ are indicated. The $\mathrm{p}$ distances were computed using the Kimura 2-parameter method and they were in the units of the number of base substitutions per site. Analyses were conducted in MEGA7. H, Holothuria; *, doubtful species; T, Tunisia; S, Spain; C-I, Canary Islands.

Description. This species has a curved U-shaped body and is between 5 and $7 \mathrm{~cm}$ long. It is usually a brownish-yellow colour (Tortonese 1965, Koehler 1969). Spicules of body have the form of large and elongated plates pierced with many holes, accompanied by irregular knobbed buttons and smooth elongated rods.

Examined material. Three specimens. Sector and location: Cape Bon (S.3/L.8). Depth: 77-145 m. Substrates: Sand (Table 1).

Distribution. Mediterranean Sea. It has been reported at many sites along the Italian coast (Koehler 1924, Tortonese 1965) and in France (Koehler 1924, Tortonese 1965), Spain (Tortonese 1965), Turkey (Özaydın et al 1995, Öztoprak 2014) and the Maltese Islands (Tanti and Schembri 2006).
Remarks. L. tergestina may have been confused with $L$. elongata (Düben and Koren, 1846), which very often has the same shape and colour. The main difference between these two species is the form of spicules.

\section{Molecular study}

Genetic analysis identified the doubtful specimens of the genus Holothuria as the species $H$. poli and $H$. tubulosa, while neighbour-joining analysis showed 28 monophyletic lineages supported by a high bootstrap value $(99 \%)$. The molecular analyses favour the subdivision of all the group taxa into two major clades: Clade I and Clade II (Fig. 4). Clade I is divided into two subclades: Clade (1) and (2), both highly supported. Clade (1) comprises only members of $H$. poli from Mediterranean locations (Tunisia and Spain), with a high bootstraps value (99\%). However, Clade 
Table 2. - Genetic distances between the three Holothuria species obtained from the phylogenetic reconstruction based on the Kimura twoparameter model (K2P).

\begin{tabular}{|c|c|c|c|c|c|}
\hline & H. tubulosa & H. sanctori & H. poli & H. lentiginosa lentiginosa & H. forskali \\
\hline H. tubulosa & - & & & & \\
\hline H. sanctori & 0.209 & - & & & \\
\hline H. poli & 0.165 & 0.223 & - & & \\
\hline H. lentiginosa lentiginosa & 0.092 & 0.192 & 0.157 & - & \\
\hline H. forskali & 0.260 & 0.189 & 0.259 & 0.249 & - \\
\hline
\end{tabular}

(2) also gains high support (95\%) and comprises both specimens of $H$. lentiginosa lentiginosa with an Atlantic origin (Canary Islands) and the Mediterranean specimens H. tubulosa (Spain and Tunisia).

Clade II, comprising the outgroup species $H$. forskali and $H$. sanctori, was separated from Clade I with high bootstraps values (99\%).

The K2P distances, based on COI sequences, are shown in Table 2. The highest divergence distance was found between $H$. poli and $H$. tubulosa $(16.5 \%)$ and the lowest between $H$. tubulosa and H. lentiginosa lentiginosa (9.2\%). The distance between $H$. poli and $H$. lentiginosa lentiginosa $(15.7 \%)$ was very close to that between $H$. poli and H. tubulosa (16.5\%).

\section{DISCUSSION}

\section{Species first record}

The Asteroidea are characterized by two new findings: Asterina pancerii and Luidia atlantidea. The starfish Asterina pancerii is an endemic species of the Mediterranean Sea (Tortonese 1965). According to Annex II of the Bern Convention in the protocol of Specially Protected Areas and Biological Diversity in the Mediterranean Sea from the Barcelona Convention and the Spanish Catalogue of Threatened Species (López-Márquez et al. 2018), it is listed as an endangered and protected species in the Mediterranean Sea. Asterina pancerii was found for the first time in Tunisia in northern inshore waters (3-5 m). However, several authors, including Ballesteros et al. (1987), Oliver et al. (1997) and López-Márquez et al. (2018), have reported that this species is typical of Posidonia oceanica ((Linnaeus) Delile, 1813) meadows. The only specimens of $A$. pancerii found so far in Tunisia were associated with Cymodocea nodosa beds.

A single Luidia atlantidea specimen was collected for the first time in the northeastern Tunisian Sea (Cape Bon, East Mediterranean Sea) by trawl-fishing gear at a depth of 65-95 m. Luidia atlantidea is an Atlantic species. It was recently found and reported for the first time in the Mediterranean Sea, in the northern Alboran Sea (western Mediterranean Sea) (Gallardo-Roldán et al. 2015), 31 individuals being collected by mechanized dredging performed at depths of between 0.9 and 11.6 m (Gallardo-Roldán et al. 2015). The present report on Luidia atlantidea is the first in Tunisia and the second in the Mediterranean Sea.

We report new findings of the ophiuroid Ophiactis virens, an eastern and northern Atlantic species. In the Mediterranean Sea, it has been so far reported only in Naples (Koehler 1924, Tortonese 1965) and in the
Turkish Levantine Sea (Özaydın et al. 1995, Öztoprak 2014). Over 184 specimens were found for the first time off the northeastern coast of Tunisia (Gulf of Tunis), at a depth of $40-60 \mathrm{~cm}$. The presence of diverse Ophiactis virens individuals over several years (from 2012 to 2015) may indicate the persistence of a local population on the shallow circalittoral Tunisian coast, as this species is well known for its asexual reproduction and fission of its body into two equal parts (Wilkie 1984). The presence of Ophiactis virens is the first to be reported in Tunisia and the third in the Mediterranean Sea.

Holothuroidea is represented by one new record for the Tunisian Sea: Leptopentacta tergestina. This sea cucumber is an endemic Mediterranean species (Koehler 1924, Tortonese 1965). In Tunisia, three individuals were found off Cape Bon (northeastern Tunisia). The Leptopentacta tergestina specimens were collected by commercial trawling at depths of between 60 and $150 \mathrm{~m}$.

Two species of the Ophiuroidea class, Astrospartus mediterraneus (Risso, 1826) and Ophiacantha setosa (Bruzelius, 1805), were found and reported for the second time in this present study after the first finding by Cherbonnier (1956). More than 30 Ophiacantha setosa specimens were found associated with the yellow gorgonian, Eunicella cavolini (Koch, 1887), close to the Algerian deep sea border. Because of its evasiveness, Ophiacantha setosa is recorded for the second time in Tunisia in this study.

\section{Species diversity}

The echinoderms recorded from northern Tunisian marine water in this study are quite diverse (45 species). Among the recorded groups, Asteroidea were the most diverse, with 15 species, followed by Ophiuroidea (10 species), Holothuroidea (9), Echinoidea (8) and Crinoidea (3). This can be explained by the techniques and gears used to sample them (hand collection, dredging, trawling and diving). Accordingly, the present research method increased the collection area by covering the marine benthic zones of the Mediterranean Sea, from the infralittoral level to the bathyal level.

Most of the new recorded species (Asterina pancerii, Luidia atlantidea and Ophiactis virens) were found in the northeastern Tunisian Sea (Cape Bon peninsula), close to the Strait of Sicily, which marks the transition between the two major western and eastern Mediterranean basins (Boudouresque 2004, Coll et al. 2010). This result confirms the importance of the Strait of Sicily as a highly primary production area with a wide range of biodiversity due its moderate depth, hy- 
drography and diversity of habitat types (Bianchi and Morri 2000, Lejeusne et al. 2010). It is one of the biodiversity hotspots in the Mediterranean Sea (Lejeusne et al. 2010, Coll et al. 2010).

A review of the relevant literature of megabenthic Tunisian inventories, including the Echinodermata phylum, by Le Danois (1925), Cherbonnier (1956), Lubet and Azouz (1969), Azouz (1971, 1973), Ben Othman (1973), Boudouresque (1997), Anonymous (1997) and El Lakhrach et al. (2012) shows the presence of 73 valid species in Tunisia. The present work increases the number of echinoderms to 77, with four new occurrences in Tunisian marine waters.

Northern Tunisia alone (from the Algerian-Tunisian border to Ras Kapudia) showed the highest number, with 69 species against 61 in the south (from Ras Kapudia to the Libyan border, including the Gulf of Gabès). However, some species present in the northern part are absent in the south and vice versa (Ben Othman 1973, Boudouresque 1997, El Lakhrach et al. 2012). Some previously recorded species were not found in the present work, since the adopted methodology and fishing gears depend on the depths frequented by fishermen.

Little research has been done on Echinodermata in deep Mediterranean waters (Koukouras et al. 2007, Coll et al. 2010), and the knowledge gap includes especially the north African coast of the Maghreb (Dauvin et al. 2013).

Echinodermata marine biodiversity along the Algerian coast, from the Moroccan border to the Tunisian border, is very low compared with that in northern $\mathrm{Tu}$ nisia, with 48 species being recorded in Algeria (Dauvin et al. 2013). According to Koukouras et al. (2007), about 144 echinoderms are known from the western Mediterranean Sea, only $53.5 \%$ of which have been found in Tunisia. On the other hand, Tunisia shares over $83.7 \%$ of a total of 91 echinoderms reported from the central Mediterranean Sea.

These findings confirm the importance of northern Tunisia area, which emerging a large number of exotic marine species and a high rate of endemic species (Ayari and Afli 2003, Ounifi Ben Amor et al. 2016). Indeed, there are more endemic species in the western part of the Mediterranean and the number of non-native species entering through the Suez Canal in the eastern basin and the Strait of Gibraltar in the western basin has increased spectacularly since the early 20 th century (Boudouresque 2004, Zenetos et al. 2010, Ben Souissi et al. 2011). Most have been introduced by maritime transport.

Overall, the present work enhances the importance of the studied fauna in northern Tunisia. To maintain the diversity of echinoderms in Tunisia's marine waters, it is necessary to promote efforts and acquire knowledge about this macrobenthic group by involving southern and eastern Tunisia.

\section{Systematic and molecular}

Systematic studies based on taxonomical and anatomical criteria have often been confusing and doubtful because of the large morphological similarity between species. Many authors have been involved in research on systematic identification and/or revision of the taxonomical status of different classes of Echinodermata and have provided molecular evidence to support their findings (Borrero-Pérez et al. 2009, Laakman et al. 2016, López-Márquez et al. 2018).

For the class Holothuroidea, Borrero-Pérez et al. (2009) evaluated the taxonomic status of some Atlanto-Mediterranean species of the subgenus Holothuria using molecular analysis and showed that the combination of the two approaches may solve the taxonomical problems associated with species identification, as was the case with $H$. (Holothuria) stellati Delle Chiaje, 1824 and $H$. tubulosa. The same authors confirmed the morphological variability in the specimens of $\mathrm{H}$. stellati and $H$. tubulosa, as mentioned in the literature, but their molecular results showed $H$. stellati to be a junior subjective synonym of $H$. tubulosa.

As regards our doubtful species, $H$. poli and $H$. tubulosa, the outcome of the phylogenetic neighbourjoining analysis showed a close relation between $H$. tubulosa and $H$. lentiginosa lentiginosa. Although $H$. poli and $H$. tubulosa are different species with different clades, the sequences of the sea cucumber $H$. lentiginosa lentiginosa were between those of the two holothurians, confirming the spicule similarity between the studied taxa.

In addition, the present study points to great morphological and molecular similarities between sea cucumbers from the Atlantic Ocean and the Mediterranean Sea. They were all characterized by elongated and twisted buttons. However, these characteristics are very common in $H$. lentiginosa lentiginosa species (Miller and Pawson 1979) and have recently been observed in $H$. poli and $H$. tubulosa individuals from the northern Tunisian Sea.

Though spicule morphology is an effective taxonomic character, it may show some overlap in some genera, such as the Holothuria genus and subgenus (Rowe 1969, Borrero-Pérez et al. 2009). This could be due to phylogenetic relationships between species that are still not well studied, or perhaps to environmental influences such as temperature, which could condition spicule formation and carbonate precipitation-another aspect worthy of study.

At present, the systematic position of the sea cucumbers of the genus Holothuria is dubious (Rowe 1969, Zavodnik 1999, Borrero-Pérez et al. 2009), so molecular and morphometric approaches are required if morphological identification is uncertain or impossible. Supported by ecological and biogeographical parameters, these techniques are a strong driving force in taxonomic study.

\section{ACKNOWLEDGEMENTS}

Special thanks are due to the fishermen for their help during the sampling along the northern coast of Tunisia. We express our sincere gratitude to Helena Ibáñez from the Department of Ecology and Hydrology (University of Murcia) and Alejandro López-López and José Galián from the Department of Zoology and 
Physical Anthropology (University of Murcia) for their help and advice on molecular analyses. We also thank the journal editor and two anonymous referees for their constructive criticism on an earlier version of this paper.

\section{REFERENCES}

Anonymous. 1997. Les invertébrés aquatiques de Tunisie II. Rapp. Ministère de 1'Environnement et de l'Aménagement du territoire, Tunis, $335 \mathrm{pp}$.

Ayari R., Afli A. 2003. Bionomie benthique du petit golfe de Tunis. Bull. Inst. Natl. Sci. Tech. Océanogr. Pêche Salammbô 30: 79-90.

Azouz A. 1971. Etude des biocénoses benthiques de la faune ichthyologique des fonds chalutables de la Tunisie. Région nord et sud-est. Ph.D. thesis. Univ. Caen, 243 pp.

Azouz A. 1973. Les fonds chalutables de la région Nord de la Tunisie. 1: Cadre physique des côtes Nord de la Tunisie. Bull. Inst. Océanogr. Pêche. Salammbô 2: 473-564.

Ballesteros M., Castello J., Gallés M. 1987. Invertebrados alguicolas marinos de las islas Pitiusas. Consell Insular d'Eivissa i Formentera. Eivissa, 96 pp.

Ben Othman S. 1973. Le Sud Tunisien (golfe de Gabès): hydrologie, sédimentologie, flore et faune. Bull. Inst. Natl. Sci. Tech. Océanogr. Pêche. Salammbô 2: 103-120.

Ben Souissi J., Diatta Y., Gargouri Ben Abdallah L., et al. 2011. Occurrence of the Monrovian surgeonfish Acanthurus monroviae (Osteichthyes: Acanthuridae) off the coast of Tunisia (central Mediterranean). Cah. Biol. Mar. 52: 331-335.

Bianchi C.N., Morri C. 2000. Marine Biodiversity of the Mediterranean Sea: Situation, Problems and Prospects for Future Research. Mar. Poll. Bull. 40: 367-376. https://doi.org/10.1016/S0025-326X(00)00027-8

Borrero-Pérez G.H., Pérez-Ruzafa A., Marcos C., et al. 2009. The taxonomic status of some Atlanto-Mediterranean species in the subgenus Holothuria (Echinodermata: Holothuroidea: Holothuriidae) based on molecular evidence. Zool. J. Linn. Soc. 157: 51-69.

https://doi.org/10.1111/j.1096-3642.2009.00529.x

Boudouresque C.F. 1997. Conclusions et recommandations (Partie I). In: La diversité biologique marine et lagunaire en Tunisie: état et connaissance actuels, recommandations pour une stratégie nationale de conservation et d'utilisation durable. Rapport RAC/ SPA - MEAT, 28 pp.

Boudouresque C.F. 2004. Marine biodiversity in the Mediterranean: status of species, populations and communities. Sci. Rep. PortCros. Natl. Park 20: 97-146.

Cherbonnier G. 1956. Les Echinodermes de Tunisie. Bull. Inst. Natl. Sci. Tech. Océanogr. Pêche. Salammbô 53: 1-23.

Clark H.L. 1918. Brittle-stars, new and old. Bull. Mus. Comp. Zool. Harvard, College LXII: 266-338.

Clark A.M., Downey M.E. 1992. Starfishes of the Atlantic. Chapman and Hall, London, 794 pp.

Coll M., Piroddi C., Steenbeek J., et al. 2010. The biodiversity of the Mediterranean Sea: estimates, patterns and threats. PLoS ONE 5: e1184236. https://doi.org/10.1371/journal.pone.0011842

Dauvin J.C., Grimes S., Bakalem A. 2013. Marine Biodiversity on the Algerian Continental Shelf (Mediterranean Sea). J. Nat. Hist. 47: 25-28. https://doi.org/10.1080/00222933.2012.752545

Deli T., Ben Attia M.H., Zitari-Chatti R., et al. 2017. Genetic and morphological divergence in the purple sea urchin Paracentrotus lividus (Echinodermata, Echinoidea) across the African Mediterranean coast. Acta Oceanol. Sin. 36: 52-66. https://doi.org/10.1007/s13131-017-1090-3

Doderlein L. 1920. Die Asteriden der Siboga-Expedition. 2. Die Gattung Luidia und ihre Stammesgeschichte. Siboga Expedition 46: 193-291.

El Lakhrach H., Hattour A., Jarbouio O., et al. 2012. Spatial distribution and abundance of the megabenthic fauna community in Gabès Gulf (Tunisia, eastern Mediterranean Sea). Mediterr. Mar. Sci. 13: 12-29. https://doi.org/10.12681/mms.19

Entrambasaguas L. 2008. Estudio faunístico y ecológico de los equinodermos del archipiélago de Cabo Verde. Ph.D. thesis. Universidad de Murcia, 301 pp.
Galán C., López-Ibor A., Templado J. 1982. Primera cita en la península Ibérica de Asterina pancerii (Gasco, 1870), (Asteroidea, Asterinidae). Actas II Simp. Iber. Estud. Bentos Mar. 3: 267-269.

Gallardo-Roldán H., Urra J., García T., et al. 2015. First record of the starfish Luidia atlantidea (Madsen, 1950) in the Mediterranean Sea, with evidence of persistent populations. Cah. Biol. Mar. 56: 263-270.

Gasco F. 1870. Intorno ad una nuova specie di Asteriscus. Bullettino dell'Associazione dei naturalisti e medici per la mutua istruzione, Napoli 6: 86-90.

Gasco F. 1876. Descrizione di alcuni Echinodermi nuovi o per la prima volta trovati nel Mediterraneo. Rend. Acad. Sci. Fis. Mat. Napoli 15: 32-41.

Gautier-Michaz M. 1958. Echinodermes. Bull. Inst. Natl. Sci. Tech. Océanogr. Pêche. Salammbô 34: 45-155.

Hattour A., Ben Mustapha K. 2015. Le golfe de Gabés: Espèces des Eaux de Ballast, Patrimoniales et Introduites: Synthèse des Campagnes 2009 et 2010 et Actualisation. Publ. Inst. Natl. Sci. Tech. Mer. 360 pp.

Kimura M. 1980. A simple method for estimating evolutionary rates of base substitutions through comparative studies of nucleotide sequences. J. Mol. Evol. 16: 111-120. https://doi.org/10.1007/BF01731581

Koehler R. 1924. Les Échinodermes des Mers d'Europe. Tomo I. Librarie Octave Doin, Paris, 362 pp.

Koehler R. 1969. Faune de France. Echinodermes, Kraus Reprint, Nendeln/ Liechtenstein, 216 pp.

Koukouras A., Sinis A.I., Bobori D., et al. 2007. The echinoderm (Deuterostomia) fauna of the Aegean Sea, and comparison with those of the neighbouring seas. J. Biol. Res 7: 67-92.

Kumar S., Stecher G., Tamura K. 2016. MEGA7: Molecular Evolutionary Genetics Analysis version 7.0 for bigger datasets. Mol. Biol. Evol. 33: 1870-1874. https://doi.org/10.1093/molbev/msw054

Laakman S., Boos K., Knebelsberger T., et al. 2016. Species identification of echinoderms from the North Sea by combining morphology and molecular data. Helgol. Mar. Res. 70: 18. https://doi.org/10.1186/s10152-016-0468-5

Le Danois E. 1925. Recherches sur les fonds chalutables des cotes de Tunisie (Croisière du chalutier «Tranche» en 1924). Bull. Inst. Natl. Sci. Tech. Océanogr. Pêche Salammbô 1: 1-56.

Lejeusne C., Chevaldonné P., Pergent-Martini C., et al. 2010. Climate change effects on a miniature ocean: the highly diverse, highly impacted Mediterranean Sea. Trends Ecol. Evol. 25: $250-260$. https://doi.org/10.1016/j.tree.2009.10.009

Lipej L., Acevedo I., Akel E.H.K., et al. 2017. New Mediterranean Biodiversity Records (March 2017). Mediterr. Mar. Sci. 18: 179-201. https://doi.org/10.12681/mms.2068

López-Márquez V., Acevedo I., Manjón-Cabeza M.E., et al. 2018. Looking for morphological evidence of cryptic species in Asterina Nardo, 1834 (Echinodermata: Asteroidea). The redescription of Asterina pancerii (Gasco, 1870) and the description of two new species. Invertebr. Syst. 32: 505-523. https://doi.org/10.1071/IS17024

Louiz I., Sellem F., Tekitek A., et al. 2003. Etude des saponines isolées d'une espèce d'Holothurie Holothuria tubulosa de la lagune de Bizerte. Bull. Inst. Natl. Sci. Tech. Océanogr. Pêche Salammbô 30: 115-120.

Lubet P., Azouz A. 1969. Etude des fonds chalutables du golfe de Tunis. Bull. Inst. Océanogr. Pêche Salammbô 1: 87-111.

Luque Á.A., Templado J. 2004. Praderas y Bosques Marinos de Andalucía. Junta de Andalucía, Sevilla.

Madsen, F.J. 1950. The echinoderms collected by the Atlantide Expedition, 1945-46. 1. Asteroidea. Atlantide Rep. 1: 167-222.

Marques V.M. 1980. Echinodermes recueillis pendant la mission «Hesperides 76» du N/O Jean Charcot. Archivos do Museu Bocage, $2^{\text {a }}$ Serie, 7: 95-10.

Mejri R., Lo Brutto S., Ben Hassine O.K., et al. 2009. A study on Pomatoschistus tortonesei Miller 1968 (Perciformes, Gobiidae) reveals the Siculo-Tunisian Strait (STS) as a breakpoint to gene flow in the Mediterranean basin. Mol. Phylogenet. Evol. 53: 596-601. https://doi.org/10.1016/j.ympev.2009.04.018

Miller J.E., Pawson D.L. 1979. A new subspecies of Holothuria lentiginosa Marenzeller from the western Atlantic Ocean. Proc. Biol. Soc. Wash. 91: 912-922.

Moreno D., Acevedo I., Templado J., et al. 2008. Asterina pancerii 
(Gasco, 1870). In: Libro Rojo de los Invertebrados de Andalucía. Consejería de Medio Ambiente, Junta de Andalucía, Sevilla, pp. 626-629.

Mortensen T. 1927. Handbook of the Echinoderms of the British Isles. Clarendon Press, Oxford, $471 \mathrm{pp}$ https://doi.org/10.5962/bhl.title.6841

Nataf G., Cherbonnier G. 1973. Les astérides d'Afrique occidentale, utilisation du microscope électronique à balayage pour une étude systématique des Luidia. Bull. Mus. Hist. Natl. Paris 81: 69-101.

Oliver J.A., Terrasa J., Guillén M. 1997. Dos nuevas citas de asterinas (Astridea, Asterinidae) en Mallorca: Asterina pancerii (Gasco, 1870) y Asterina phylactica (Emson y Crump, 1979). Boll. Soc. Hist. Nat. Balears 4: 103-107.

Ounifi Ben Amor K., Rifi M., Ghanem R., et al. 2016. Update of alien fauna and new records from Tunisian marine waters. Mediterr. Mar. Sci. 17: 124-143. https://doi.org/10.12681/mms.137.

Özaydın O., Katağan T., Ünsal S. 1995. The Echinoderms of the Turkish seas. Isr. J. Zool. 41: 57-68.

Öztoprak B., Doğan A., Dağli E. 2014. Checklist of Echinodermata from the coasts of Turkey. Turk. J. Zool. 38: 892-900. https://doi.org/10.3906/zoo-1405-82

Palumbi S.R. 1996. Nucleic acids II: the polymerase chain reaction. In: Hillis D.M., Moritz C.M., et al. (eds), Molecular systematics, Sinauer Associates, Inc., Sunderland, MA. pp. 205-247.

Panning A. 1949. Versuch einer neuordnung der familie Cucumariidae (Holothurioidea, Dendrochirota). Zool. Jahrb. Abt. Syst. Okol. Geogr. Tiere 78: 404-470.

Pérez-Losada M., Nolte M.J., Crandall K.A et al. 2007. Testing hypotheses of population structuring in the Northeast Atlantic Ocean and Mediterranean Sea using the common cuttlefish Sepia officinalis. Mol. Ecol. 16: 2667-2679. https://doi.org/10.1111/j.1365-294X.2007.03333.x

Pérez-Ruzafa A., López-Ibor A. 1988. Echinoderm fauna from the south-western Medierranean, Biogeographic relationships. In: Burke R.D., Mladenov P.V., et al. (eds) Echinoderm biology. AA Balkema, Rotterdam, pp. 355-362.

Perrier E, 1886. Les explorations sous-marines. Librairie Hachette et Cie., Paris, $352 \mathrm{pp}$

Rowe F.W.E. 1969. A review of the family Holothuriidae (Holothurioidea: Aspidochirotida). Bull. Br. Mus. (Nat. Hist.) Zool. 18: 119-170.

https://doi.org/10.5962/bhl.part.18419

Sambrook E., Fritsch F., Maniatis T. 1989. Molecular cloning. Cold Spring Harbour Press, NY.

Sars M. 1859. Bidrag til Kundskaben om Middelhavets Littoral-
Fauna. Nyt Mag. Naturv. 10: 57-155.

Sellem F., Langar H., El Abed A. 2001. Ecobiology and sustainable management of sea urchin in the southeast of the gulf of Tunis. In: Ozhan E. (eds) Proc. Fifth Int. Conf. Mediterr. Coast. Environ., MEDCOAST 01, Hammamet, Tunisia, 23-27 October 2001, 2: 833-837.

Simroth H. 1876. Anatomie und Schizogonie der Ophiactis virens Sars. Ein Beitrag zur Kenntniss der Echinodermen. Z. Wiss. Zool. 27: 417-485.

Tanti C.M., Schembri P.J. 2006. A synthesis of the echinoderm fauna of the Maltese islands. J. Mar. Biol. UK 86: 163-165. https://doi.org/10.1017/S0025315406012987

Tortonese E. 1965. Echinodermata. Fauna d'Italia. Vol. 6. Edizioni Calderini, Bologna, 422 pp.

Uthicke S., Purcell S., Blockmans B. 2005. Natural hybridization does not dissolve species boundaries in commercially important sea cucumbers. Biol. J. Linnean Soc. 85: 261-270. https://doi.org/10.1111/j.1095-8312.2005.00489.x

Wilkie I.C. 1984. Variable tensility in echinoderm collagenous tissues: a review. Mar. Freshw. Behav. Physiol 11: 1-34. https://doi.org/10.1080/10236248409387032

WoRMS Editorial Board. 2019. World Register of Marine Species. Accessed 7/5/2019. Available at http://www.marinespecies.org

Zavodnik D. 1999. Echinodermata of Kastela Bay (Adriatic Sea, Croatia). Acta Adriat. 40: 45-54

Zenetos A. 2010. Trend in aliens species in the Mediterranean. An answer to Galil, 2009 "Taking stock: Inventory of alien species in the Mediterranean Sea". Biol. Invasions 12: 3379-3381. https://doi.org/10.1007/s10530-009-9679-x

Zitari-Chatti R., Chatti N., Fulgione D., et al. 2009. Mitochondrial DNA variation in the caramote prawn Penaeus (Melicertus) kerathurus across a transition zone in the Mediterranean Sea. Genetica 136: 439-447.

https://doi.org/10.1007/s10709-008-9344-9

\section{SUPPLEMENTARY MATERIAL}

The following supplementary material is available through the online version of this article and at the following link:

http://scimar.icm.csic.es/scimar/supplm/sm04899esm.pdf

Table S1. - Collection sites and sampling methods of the echinoderms from different localities of northern Tunisia.

Table S2. - Samples name's and diameters. With the habitat type, depth (maximum and minimum) and date of collection of each sample. 
Scientia Marina 83(3)

September 2019, S1-S21, Barcelona (Spain)

ISSN-L: 0214-8358

\section{Checklist with first records for the Echinoderms of northern Tunisia (central Mediterranean Sea)}

Hayfa Chammem, Jamila Ben Souissi, Angel Pérez-Ruzafa

Supplementary material 
Table S1. - Collection sites and sampling methods of the echinoderms from different localities of northern Tunisia.

\begin{tabular}{|c|c|c|c|c|c|c|}
\hline \multirow{2}{*}{$\begin{array}{l}\text { Sectors } \\
\text { (S) }\end{array}$} & \multirow{2}{*}{$\begin{array}{l}\text { Locations } \\
\text { (L) }\end{array}$} & \multirow{2}{*}{ No. } & \multicolumn{2}{|c|}{ Geographic coordinates } & \multirow{2}{*}{ Sampling methods } & \multirow{2}{*}{ Year } \\
\hline & & & Latitude & Longitude & & \\
\hline \multirow[t]{55}{*}{ S.1 } & L.1 & 1 & $37.3614^{\circ} \mathrm{S}$ & $8.5078^{\circ} \mathrm{W}$ & Hand collection & 2016 \\
\hline & & 2 & $37.2458^{\circ} \mathrm{S}$ & $8.6238^{\circ} \mathrm{W}$ & Experimental Dredge & 2016 \\
\hline & & 3 & $37.0633^{\circ} \mathrm{S}$ & $8.6267^{\circ} \mathrm{W}$ & Experimental Dredge & 2016 \\
\hline & & 4 & $37.0556^{\circ} \mathrm{S}$ & $8.6383^{\circ} \mathrm{W}$ & Experimental Dredge & 2016 \\
\hline & & 5 & $37.0771^{\circ} \mathrm{S}$ & $8.6440^{\circ} \mathrm{W}$ & Experimental Dredge & 2016 \\
\hline & & 6 & $37.0882^{\circ} \mathrm{S}$ & $8.6443^{\circ} \mathrm{W}$ & Experimental Dredge & 2016 \\
\hline & & 7 & $37.0864^{\circ} \mathrm{S}$ & $8.6459^{\circ} \mathrm{W}$ & Experimental Dredge & 2016 \\
\hline & & 8 & $37.0582^{\circ} \mathrm{S}$ & $8.7455^{\circ} \mathrm{W}$ & Experimental Dredge & 2016 \\
\hline & & 9 & $36.9644^{\circ} \mathrm{S}$ & $8.7551^{\circ} \mathrm{W}$ & Diving & 2016 \\
\hline & & 10 & $36.9591^{\circ} \mathrm{S}$ & $8.7554^{\circ} \mathrm{W}$ & Diving & 2016 \\
\hline & & 11 & $36.9635^{\circ} \mathrm{S}$ & $8.7555^{\circ} \mathrm{W}$ & Diving & 2016 \\
\hline & & 12 & $36.9592^{\circ} \mathrm{S}$ & $8.7558^{\circ} \mathrm{W}$ & Diving & 2016 \\
\hline & & 13 & $36.9657^{\circ} \mathrm{S}$ & $8.7572^{\circ} \mathrm{W}$ & Diving & 2016 \\
\hline & & 14 & $37.1157^{\circ} \mathrm{S}$ & $8.7579^{\circ} \mathrm{W}$ & Benthic Trawling & 2016 \\
\hline & & 15 & $37.1226^{\circ} \mathrm{S}$ & $8.7839^{\circ} \mathrm{W}$ & Benthic Trawling & 2016 \\
\hline & & 16 & $37.1219^{\circ} \mathrm{S}$ & $8.7847^{\circ} \mathrm{W}$ & Benthic Trawling & 2016 \\
\hline & & 17 & $37.1312^{\circ} \mathrm{S}$ & $8.7909^{\circ} \mathrm{W}$ & Benthic Trawling & 2016 \\
\hline & & 18 & $37.0655^{\circ} \mathrm{S}$ & $8.8366^{\circ} \mathrm{W}$ & Benthic Trawling & 2016 \\
\hline & & 19 & $37.5643^{\circ} \mathrm{S}$ & $8.8978^{\circ} \mathrm{W}$ & Benthic Trawling & 2016 \\
\hline & & 20 & $37.4715^{\circ} \mathrm{S}$ & $8.8992^{\circ} \mathrm{W}$ & Benthic Trawling & 2016 \\
\hline & & 21 & $37.1731^{\circ} \mathrm{S}$ & $8.9538^{\circ} \mathrm{W}$ & Benthic Trawling & 2016 \\
\hline & & 22 & $37.2006^{\circ} \mathrm{S}$ & $8.9716^{\circ} \mathrm{W}$ & Benthic Trawling & 2016 \\
\hline & & 23 & $37.5015^{\circ} \mathrm{S}$ & $8.8893^{\circ} \mathrm{W}$ & Benthic Trawling & 2016 \\
\hline & & 24 & $37.4462^{\circ} \mathrm{S}$ & $8.6197^{\circ} \mathrm{W}$ & Benthic Trawling & 2016 \\
\hline & & 25 & $37.5664^{\circ} \mathrm{S}$ & $8.9713^{\circ} \mathrm{W}$ & Benthic Trawling & 2016 \\
\hline & L. 2 & 1 & $37,4189^{\circ} \mathrm{S}$ & $8,7187^{\circ} \mathrm{W}$ & Benthic Trawling & 2016 \\
\hline & & 2 & $37,4550^{\circ} \mathrm{S}$ & $9,0303^{\circ} \mathrm{W}$ & Benthic Trawling & 2016 \\
\hline & & 3 & $37,3833^{\circ} \mathrm{S}$ & $9,1417^{\circ} \mathrm{W}$ & Benthic Trawling & 2016 \\
\hline & & 4 & $37.3799^{\circ} \mathrm{S}$ & $9.0167^{\circ} \mathrm{W}$ & Benthic Trawling & 2016 \\
\hline & & 5 & $37,3375^{\circ} \mathrm{S}$ & $9,1923^{\circ} \mathrm{W}$ & Benthic Trawling & 2016 \\
\hline & & 6 & $37,3675^{\circ} \mathrm{S}$ & $9,3199^{\circ} \mathrm{W}$ & Benthic Trawling & 2016 \\
\hline & & 7 & $37,1506^{\circ} \mathrm{S}$ & $9,8706^{\circ} \mathrm{W}$ & Experimental Dredge & 2015 \\
\hline & & 8 & $37,1426^{\circ} \mathrm{S}$ & $9,8726^{\circ} \mathrm{W}$ & Experimental Dredge & 2015 \\
\hline & & 9 & $37,1470^{\circ} \mathrm{S}$ & $9,8740^{\circ} \mathrm{W}$ & Experimental Dredge & 2015 \\
\hline & & 10 & $37,2716^{\circ} \mathrm{S}$ & $9,8793^{\circ} \mathrm{W}$ & Hand collection & 2013 \\
\hline & & 11 & $37,2717^{\circ} \mathrm{S}$ & $9,8795^{\circ} \mathrm{W}$ & Hand collection & 2013 \\
\hline & & 12 & $37,2715^{\circ} \mathrm{S}$ & $9,8843^{\circ} \mathrm{W}$ & Hand collection & 2013 \\
\hline & & 13 & $37,6016^{\circ} \mathrm{S}$ & $9,9434^{\circ} \mathrm{W}$ & Benthic Trawling & 2016 \\
\hline & & 14 & $37,6445^{\circ} \mathrm{S}$ & $9,9682^{\circ} \mathrm{W}$ & Benthic Trawling & 2016 \\
\hline & & 15 & $37,3217^{\circ} \mathrm{S}$ & $9,9670^{\circ} \mathrm{W}$ & Benthic Trawling & 2016 \\
\hline & & 16 & $37.4550^{\circ} \mathrm{S}$ & $9.0303^{\circ} \mathrm{W}$ & Benthic Trawling & 2016 \\
\hline & & 17 & $37.1426^{\circ} \mathrm{S}$ & $9.8726^{\circ} \mathrm{W}$ & Experimental Dredge & 2016 \\
\hline & & 18 & $37.3833^{\circ} \mathrm{S}$ & $9.1417^{\circ} \mathrm{W}$ & Experimental Dredge & 2016 \\
\hline & & 19 & $37,3934^{\circ} \mathrm{S}$ & $10,0499^{\circ} \mathrm{W}$ & Benthic Trawling & 2016 \\
\hline & & 20 & $37,6782^{\circ} \mathrm{S}$ & $10,0633^{\circ} \mathrm{W}$ & Benthic Trawling & 2016 \\
\hline & & 21 & $37.5448^{\circ} \mathrm{S}$ & $10.1377^{\circ} \mathrm{W}$ & Benthic Trawling & 2016 \\
\hline & & 22 & $37.5598^{\circ} \mathrm{S}$ & $10.0670^{\circ} \mathrm{W}$ & Benthic Trawling & 2016 \\
\hline & & 23 & $37.6205^{\circ} \mathrm{S}$ & $10.2040^{\circ} \mathrm{W}$ & Benthic Trawling & 2016 \\
\hline & & 24 & $37.6124^{\circ} \mathrm{S}$ & $10.2516^{\circ} \mathrm{W}$ & Benthic Trawling & 2016 \\
\hline & & 25 & $37.4995^{\circ} \mathrm{S}$ & $10.2526^{\circ} \mathrm{W}$ & Benthic Trawling & 2016 \\
\hline & & 26 & $37.5643^{\circ} \mathrm{S}$ & $10.2075^{\circ} \mathrm{W}$ & Benthic Trawling & 2016 \\
\hline & & 27 & $37.6191^{\circ} \mathrm{S}$ & $10.3120^{\circ} \mathrm{W}$ & Benthic Trawling & 2016 \\
\hline & & 28 & $37.4431^{\circ} \mathrm{S}$ & $10.3229^{\circ} \mathrm{W}$ & Benthic Trawling & 2016 \\
\hline & & 29 & $37.5757^{\circ} \mathrm{S}$ & $10.3278^{\circ} \mathrm{W}$ & Benthic Trawling & 2016 \\
\hline & & 30 & $37.5236^{\circ} \mathrm{S}$ & $10.3729^{\circ} \mathrm{W}$ & Benthic Trawling & 2016 \\
\hline S. 2 & L. 3 & 1 & $36.8405^{\circ} \mathrm{S}$ & $10.3292^{\circ} \mathrm{W}$ & Hand collection & 2012 \\
\hline & & 2 & $36.8441^{\circ} \mathrm{S}$ & $10.3323^{\circ} \mathrm{W}$ & Hand collection & 2013 \\
\hline & L.4 & 1 & $36.8071^{\circ} \mathrm{S}$ & $10.3066^{\circ} \mathrm{W}$ & Experimental Dredge & 2012 \\
\hline & & 2 & $36.8067^{\circ} \mathrm{S}$ & $10.3075^{\circ} \mathrm{W}$ & Experimental Dredge & 2013 \\
\hline & & 3 & $36.8220^{\circ} \mathrm{S}$ & $10.3137^{\circ} \mathrm{W}$ & Experimental Dredge & 2013 \\
\hline & L. 5 & 1 & $36.7880^{\circ} \mathrm{S}$ & $10.2878^{\circ} \mathrm{W}$ & Hand collection & 2012 \\
\hline & & 2 & $37.2717^{\circ} \mathrm{S}$ & $9.8795^{\circ} \mathrm{W}$ & Hand collection & 2013 \\
\hline & L. 6 & 1 & $36.8159^{\circ} \mathrm{S}$ & $10.5611^{\circ} \mathrm{W}$ & Hand collection & 2013 \\
\hline S.3 & L. 7 & 1 & $37.0762^{\circ} \mathrm{S}$ & $11.0526^{\circ} \mathrm{W}$ & Diving & 2014 \\
\hline & & 2 & $37.0760^{\circ} \mathrm{S}$ & $11.0530^{\circ} \mathrm{W}$ & Hand collection & 2015 \\
\hline & & 3 & $37.0758^{\circ} \mathrm{S}$ & $11.0539^{\circ} \mathrm{W}$ & Hand collection & 2016 \\
\hline & L. 8 & 1 & $36.5593^{\circ} \mathrm{S}$ & $10.9349^{\circ} \mathrm{W}$ & Benthic Trawling & 2015 \\
\hline & & 2 & $36.5507^{\circ} \mathrm{S}$ & $11.0163^{\circ} \mathrm{W}$ & Benthic Trawling & 2015 \\
\hline & & 3 & $36.6083^{\circ} \mathrm{S}$ & $11.0249^{\circ} \mathrm{W}$ & Benthic Trawling & 2015 \\
\hline & & 4 & $36.6683^{\circ} \mathrm{S}$ & $11.0667^{\circ} \mathrm{W}$ & Benthic Trawling & 2015 \\
\hline & & 5 & $36.6433^{\circ} \mathrm{S}$ & $11.0899^{\circ} \mathrm{W}$ & Benthic Trawling & 2015 \\
\hline & & 6 & $36.7500^{\circ} \mathrm{S}$ & $11.1017^{\circ} \mathrm{W}$ & Benthic Trawling & 2015 \\
\hline & & 7 & $36.8138^{\circ} \mathrm{S}$ & $11.1609^{\circ} \mathrm{W}$ & Benthic Trawling & 2015 \\
\hline & & 8 & $36.6267^{\circ} \mathrm{S}$ & $11.1784^{\circ} \mathrm{W}$ & Benthic Trawling & 2015 \\
\hline & & 9 & $36.6507^{\circ} \mathrm{S}$ & $11.1812^{\circ} \mathrm{W}$ & Benthic Trawling & 2015 \\
\hline & & 10 & $36.7463^{\circ} \mathrm{S}$ & $11.1583^{\circ} \mathrm{W}$ & Benthic Trawling & 2015 \\
\hline
\end{tabular}




\begin{tabular}{|c|c|c|c|c|c|c|}
\hline \multirow{2}{*}{$\begin{array}{l}\text { Sectors } \\
\text { (S) }\end{array}$} & \multirow{2}{*}{$\begin{array}{l}\text { Locations } \\
\text { (L) }\end{array}$} & \multirow[b]{2}{*}{ No. } & \multicolumn{2}{|c|}{ Geographic coordinates } & \multirow[b]{2}{*}{ Sampling methods } & \multirow[b]{2}{*}{ Year } \\
\hline & & & Latitude & Longitude & & \\
\hline \multirow[t]{20}{*}{ S.3 } & L. 8 & 11 & $36.6132^{\circ} \mathrm{S}$ & $11.3574^{\circ} \mathrm{W}$ & Benthic Trawling & 2015 \\
\hline & & 12 & $36.6322^{\circ} \mathrm{S}$ & $11.1861^{\circ} \mathrm{W}$ & Benthic Trawling & 2016 \\
\hline & & 13 & $36.8645^{\circ} \mathrm{S}$ & $11.1996^{\circ} \mathrm{W}$ & Benthic Trawling & 2016 \\
\hline & & 14 & $36.7517^{\circ} \mathrm{S}$ & $11.2083^{\circ} \mathrm{W}$ & Benthic Trawling & 2016 \\
\hline & & 15 & $36.8445^{\circ} \mathrm{S}$ & $11.2182^{\circ} \mathrm{W}$ & Benthic Trawling & 2016 \\
\hline & & 16 & $36.8812^{\circ} \mathrm{S}$ & $11.2269^{\circ} \mathrm{W}$ & Benthic Trawling & 2016 \\
\hline & & 17 & $36.6183^{\circ} \mathrm{S}$ & $11.2194^{\circ} \mathrm{W}$ & Benthic Trawling & 2016 \\
\hline & & 18 & $36.9323^{\circ} \mathrm{S}$ & $11.2289^{\circ} \mathrm{W}$ & Benthic Trawling & 2016 \\
\hline & & 19 & $36.7099^{\circ} \mathrm{S}$ & $11.2233^{\circ} \mathrm{W}$ & Benthic Trawling & 2016 \\
\hline & & 20 & $36.6767^{\circ} \mathrm{S}$ & $11.2233^{\circ} \mathrm{W}$ & Benthic Trawling & 2016 \\
\hline & & 21 & $36.8087^{\circ} \mathrm{S}$ & $11.2505^{\circ} \mathrm{W}$ & Benthic Trawling & 2016 \\
\hline & & 22 & $36.8367^{\circ} \mathrm{S}$ & $11.2583^{\circ} \mathrm{W}$ & Benthic Trawling & 2016 \\
\hline & & 23 & $36.5467^{\circ} \mathrm{S}$ & $11.2517^{\circ} \mathrm{W}$ & Benthic Trawling & 2016 \\
\hline & & 24 & $36.8752^{\circ} \mathrm{S}$ & $11.2653^{\circ} \mathrm{W}$ & Benthic Trawling & 2016 \\
\hline & & 25 & $36.6667^{\circ} \mathrm{S}$ & $11.2683^{\circ} \mathrm{W}$ & Benthic Trawling & 2016 \\
\hline & & 26 & $36.5957^{\circ} \mathrm{S}$ & $11.2796^{\circ} \mathrm{W}$ & Benthic Trawling & 2016 \\
\hline & & 27 & $36.2534^{\circ} \mathrm{S}$ & $11.2768^{\circ} \mathrm{W}$ & Benthic Trawling & 2016 \\
\hline & & 28 & $36.8078^{\circ} \mathrm{S}$ & $11.3333^{\circ} \mathrm{W}$ & Benthic Trawling & 2016 \\
\hline & & 29 & $36.7713^{\circ} \mathrm{S}$ & $11.3447^{\circ} \mathrm{W}$ & Benthic Trawling & 2016 \\
\hline & & 30 & $36.6132^{\circ} \mathrm{S}$ & $11.3574^{\circ} \mathrm{W}$ & Benthic Trawling & 2016 \\
\hline
\end{tabular}

Table S2. - Samples name's and diameters. With the habitat type, depth (maximum and minimum) and date of collection of each sample.

\begin{tabular}{|c|c|c|c|c|c|c|c|}
\hline $\begin{array}{c}\text { Depth - Min } \\
(\mathrm{m})\end{array}$ & $\begin{array}{c}\text { Depth-Max } \\
(\mathrm{m})\end{array}$ & Habitats & Abr & Species & $\begin{array}{c}\text { Diameter } \\
(\mathrm{cm})\end{array}$ & Year & Month \\
\hline 0.45 & 0.55 & Rock & Asgib & Asterina gibbosa & 5 & 2013 & \\
\hline 0.52 & 0.65 & Rock & Asgib & Asterina gibbosa & 3 & 2013 & \\
\hline 0.45 & 0.8 & Rock & Asgib & Asterina gibbosa & 4 & 2013 & \\
\hline 0.45 & 0.8 & Rock & Asgib & Asterina gibbosa & 4.5 & 2013 & \\
\hline 0.45 & 0.8 & Rock & Asgib & Asterina gibbosa & 4 & 2013 & \\
\hline 0.45 & 0.8 & Algea & Asgib & Asterina gibbosa & 5 & 2013 & \\
\hline 0.45 & 0.8 & Algea & Asgib & Asterina gibbosa & 3.5 & 2013 & \\
\hline 0.45 & 0.8 & Algea & Asgib & Asterina gibbosa & 2 & 2013 & \\
\hline 0.45 & 0.8 & Algea & Asgib & Asterina gibbosa & 2.5 & 2013 & \\
\hline 0.45 & 0.8 & Algea & Asgib & Asterina gibbosa & 3 & 2013 & \\
\hline 0.45 & 0.8 & Algea & Asgib & Asterina gibbosa & 1.3 & 2013 & \\
\hline 0.45 & 0.8 & Algea & Asgib & Asterina gibbosa & 1.5 & 2013 & \\
\hline 0.45 & 0.8 & Algea & Asgib & Asterina gibbosa & 1.7 & 2013 & \\
\hline 2 & 5 & Cymodocea & Aspan & Asterina pancerii & 1 & 2013 & \\
\hline 98 & 105 & Sand & Asara & Astropecten aranciacus & 5 & 2015 & October \\
\hline 98 & 105 & Sand & Asara & Astropecten aranciacus & 5 & 2015 & October \\
\hline 98 & 105 & Sand & Asara & Astropecten aranciacus & 5 & 2015 & October \\
\hline 98 & 105 & Sand & Asara & Astropecten aranciacus & 5 & 2015 & October \\
\hline 91 & 95 & Sand & Asara & Astropecten aranciacus & 5 & 2015 & October \\
\hline 91 & 95 & Sand & Asara & Astropecten aranciacus & 7 & 2015 & October \\
\hline 91 & 95 & Sand & Asara & Astropecten aranciacus & 7 & 2015 & October \\
\hline 91 & 95 & Sand & Asara & Astropecten aranciacus & 7 & 2015 & October \\
\hline 91 & 95 & Sand & Asara & Astropecten aranciacus & 7 & 2015 & October \\
\hline 91 & 95 & Sand & Asara & Astropecten aranciacus & 7 & 2015 & October \\
\hline 91 & 95 & Sand & Asara & Astropecten aranciacus & 7 & 2015 & October \\
\hline 91 & 95 & Sand & Asara & Astropecten aranciacus & 7 & 2015 & October \\
\hline 91 & 95 & Sand & Asara & Astropecten aranciacus & 8 & 2015 & October \\
\hline 90 & 110 & Mud-Sand & Asara & Astropecten aranciacus & 8 & 2015 & December \\
\hline 90 & 110 & Mud-Sand & Asara & Astropecten aranciacus & 8 & 2015 & December \\
\hline 90 & 110 & Mud-Sand & Asara & Astropecten aranciacus & 8 & 2015 & December \\
\hline 90 & 110 & Mud-Sand & Asara & Astropecten aranciacus & 12 & 2015 & December \\
\hline 80 & 113 & Mud & Asara & Astropecten aranciacus & 12 & 2016 & March \\
\hline 90 & 120 & Sand & Asara & Astropecten aranciacus & 12 & 2016 & March \\
\hline 50 & 53 & Sand & Asara & Astropecten aranciacus & 14 & 2016 & August \\
\hline 50 & 53 & Sand & Asara & Astropecten aranciacus & 14 & 2016 & August \\
\hline 92 & 96 & Mud & Asara & Astropecten aranciacus & 14 & 2016 & August \\
\hline 152 & 177 & Sand & Asara & Astropecten aranciacus & 16 & 2016 & April \\
\hline 51 & 58 & Mud & Asara & Astropecten aranciacus & 16 & 2016 & June \\
\hline 2 & 5 & Sand & Asbis & Astropecten bispinosus & 6 & 2013 & \\
\hline 2 & 5 & Sand & Asbis & Astropecten bispinosus & 6 & 2013 & \\
\hline 2 & 5 & Sand & Asbis & Astropecten bispinosus & 7 & 2013 & \\
\hline 2 & 5 & Sand & Asbis & Astropecten bispinosus & 7 & 2013 & \\
\hline 2 & 5 & Sand & Asbis & Astropecten bispinosus & 8 & 2013 & \\
\hline 2 & 5 & Sand & Asbis & Astropecten bispinosus & 8 & 2013 & \\
\hline 10 & 35 & Sand & Asbis & Astropecten bispinosus & 7 & 2014 & \\
\hline 10 & 35 & Sand & Asbis & Astropecten bispinosus & 8 & 2014 & \\
\hline 10 & 35 & Sand & Asbis & Astropecten bispinosus & 8 & 2014 & \\
\hline 0 & 1 & Sand & Asbis & Astropecten bispinosus & 8 & 2014 & \\
\hline
\end{tabular}




\begin{tabular}{|c|c|c|c|c|c|c|c|}
\hline $\begin{array}{l}\text { Depth - Min } \\
(\mathrm{m})\end{array}$ & $\begin{array}{c}\text { Depth-Max } \\
(\mathrm{m})\end{array}$ & Habitats & Abr & Species & $\begin{array}{l}\text { Diameter } \\
(\mathrm{cm})\end{array}$ & Year & Month \\
\hline 0 & 1 & Sand & Asbis & Astropecten bispinosus & 8 & 2014 & \\
\hline 98 & 105 & Sand & Asirr & Astropecten irregularis & 5 & 2015 & October \\
\hline 98 & 105 & Sand & Asirr & Astropecten irregularis & 5 & 2015 & October \\
\hline 68 & 94 & Mud-Sand & Asirr & Astropecten irregularis & 5 & 2015 & October \\
\hline 68 & 94 & Mud-Sand & Asirr & Astropecten irregularis & 5 & 2015 & October \\
\hline 91 & 95 & Sand & Asirr & Astropecten irregularis & 5 & 2015 & October \\
\hline 91 & 95 & Sand & Asirr & Astropecten irregularis & 5 & 2015 & October \\
\hline 91 & 95 & Sand & Asirr & Astropecten irregularis & 5 & 2015 & October \\
\hline 120 & 145 & Mud & Asirr & Astropecten irregularis & 5 & 2015 & December \\
\hline 120 & 145 & Mud & Asirr & Astropecten irregularis & 5 & 2015 & December \\
\hline 79 & 80 & Sand & Asirr & Astropecten irregularis & 5 & 2016 & March \\
\hline 100 & 115 & Sand & Asirr & Astropecten irregularis & 5 & 2016 & March \\
\hline 100 & 115 & Sand & Asirr & Astropecten irregularis & 5 & 2016 & March \\
\hline 100 & 115 & Sand & Asirr & Astropecten irregularis & 5 & 2016 & March \\
\hline 50 & 65 & Sand & Asirr & Astropecten irregularis & 6 & 2016 & March \\
\hline 50 & 65 & Sand & Asirr & Astropecten irregularis & 6 & 2016 & March \\
\hline 50 & 65 & Sand & Asirr & Astropecten irregularis & 6 & 2016 & March \\
\hline 50 & 103 & Sand & Asirr & Astropecten irregularis & 6 & 2016 & August \\
\hline 50 & 103 & Sand & Asirr & Astropecten irregularis & 6 & 2016 & August \\
\hline 101 & 105 & Sand & Asirr & Astropecten irregularis & 6 & 2016 & August \\
\hline 101 & 105 & Sand & Asirr & Astropecten irregularis & 6 & 2016 & August \\
\hline 101 & 105 & Sand & Asirr & Astropecten irregularis & 6 & 2016 & August \\
\hline 185 & 220 & Sand & Asirr & Astropecten irregularis & 6 & 2016 & April \\
\hline 185 & 220 & Sand & Asirr & Astropecten irregularis & 6 & 2016 & April \\
\hline 51 & 58 & Mud & Asirr & Astropecten irregularis & 6 & 2016 & June \\
\hline 130 & 140 & Sand & Asirr & Astropecten irregularis & 6 & 2016 & June \\
\hline 81 & 100 & Sand & Asirr & Astropecten irregularis & 6 & 2016 & June \\
\hline 81 & 100 & Sand & Asirr & Astropecten irregularis & 6 & 2016 & June \\
\hline 90 & 160 & Mud-Sand & Asirr & Astropecten irregularis & 6 & 2016 & July \\
\hline 176 & 179 & Mud-Sand & Asirr & Astropecten irregularis & 7 & 2016 & July \\
\hline 181 & 186 & Mud-Sand & Asirr & Astropecten irregularis & 7 & 2016 & July \\
\hline 181 & 186 & Mud-Sand & Asirr & Astropecten irregularis & 7 & 2016 & July \\
\hline 179 & 185 & Sand & Asirr & Astropecten irregularis & 7 & 2016 & July \\
\hline 181 & 190 & Sand & Asirr & Astropecten irregularis & 7 & 2016 & July \\
\hline 3 & 5 & Sand & Asjon & Astropecten jonstoni & 6 & 2013 & 2013 \\
\hline 190 & 230 & Sand & Asirr & Astropecten irregularis & 12 & 2016 & June \\
\hline 180 & 200 & Sand & Asirr & Astropecten irregularis & 14 & 2016 & June \\
\hline 185 & 220 & Sand & Anpla & Anseropoda placenta & 12 & 2016 & April \\
\hline 98 & 105 & Sand & Chlon & Chataster longipe & 6 & 2015 & October \\
\hline 70 & 95 & Rock & Chlon & Chataster longipe & 5 & 2016 & June \\
\hline 70 & 95 & Rock & Chlon & Chataster longipe & 5 & 2016 & June \\
\hline 70 & 95 & Rock & Chlon & Chataster longipe & 6 & 2016 & June \\
\hline 72 & 90 & Rock & Chlon & Chataster longipe & 6 & 2016 & September \\
\hline 72 & 90 & Rock & Chlon & Chataster longipe & 6 & 2016 & September \\
\hline 72 & 90 & Rock & Chlon & Chataster longipe & 6 & 2016 & September \\
\hline 72 & 90 & Rock & Chlon & Chataster longipe & 7 & 2016 & September \\
\hline 72 & 90 & Rock & Chlon & Chataster longipe & 7 & 2016 & September \\
\hline 78 & 84 & Rock & Chlon & Chataster longipe & 7 & 2016 & September \\
\hline 78 & 84 & Rock & Chlon & Chataster longipe & 7 & 2016 & September \\
\hline 78 & 84 & Rock & Chlon & Chataster longipe & 8 & 2016 & September \\
\hline 135 & 170 & Sand & Chlon & Chataster longipe & 8 & 2016 & September \\
\hline 135 & 170 & Sand & Chlon & Chataster longipe & 8 & 2016 & September \\
\hline 135 & 170 & Sand & Chlon & Chataster longipe & 8 & 2016 & September \\
\hline 160 & 161 & Sand & Chlon & Chataster longipe & 8 & 2016 & July \\
\hline 70 & 85 & Rock & Chlon & Chataster longipe & 8 & 2016 & July \\
\hline 70 & 85 & Rock & Chlon & Chataster longipe & 8 & 2016 & July \\
\hline 70 & 85 & Rock & Chlon & Chataster longipe & 10 & 2016 & July \\
\hline 50 & 51 & Rock & Chlon & Chataster longipe & 10 & 2016 & July \\
\hline 50 & 51 & Rock & Chlon & Chataster longipe & 12 & 2016 & July \\
\hline 50 & 51 & Rock & Coten & Concinasteria tenuispina & 7 & 2016 & August \\
\hline 0 & 0.55 & Rock & Coten & Concinasteria tenuispina & 12 & 2013 & 2013 \\
\hline 85 & 98 & Mud & Ecsep & Echinaster sepositus & 4 & 2015 & October \\
\hline 85 & 98 & Mud & Ecsep & Echinaster sepositus & 4 & 2015 & October \\
\hline 85 & 98 & Mud & Ecsep & Echinaster sepositus & 4 & 2015 & October \\
\hline 85 & 98 & Mud & Ecsep & Echinaster sepositus & 4 & 2015 & October \\
\hline 98 & 105 & Sand & Ecsep & Echinaster sepositus & 5 & 2015 & October \\
\hline 98 & 105 & Sand & Ecsep & Echinaster sepositus & 5 & 2015 & October \\
\hline 98 & 105 & Sand & Ecsep & Echinaster sepositus & 5 & 2015 & October \\
\hline 68 & 94 & Mud-Sand & Ecsep & Echinaster sepositus & 5 & 2015 & October \\
\hline 68 & 94 & Mud-Sand & Ecsep & Echinaster sepositus & 5 & 2015 & October \\
\hline 68 & 94 & Mud-Sand & Ecsep & Echinaster sepositus & 5 & 2015 & October \\
\hline 68 & 94 & Mud-Sand & Ecsep & Echinaster sepositus & 5 & 2015 & October \\
\hline 68 & 94 & Mud-Sand & Ecsep & Echinaster sepositus & 5 & 2015 & October \\
\hline 68 & 94 & Mud-Sand & Ecsep & Echinaster sepositus & 5 & 2015 & October \\
\hline 91 & 95 & Sand & Ecsep & Echinaster sepositus & 5 & 2015 & October \\
\hline 91 & 95 & Sand & Ecsep & Echinaster sepositus & 6 & 2015 & October \\
\hline 91 & 95 & Sand & Ecsep & Echinaster sepositus & 6 & 2015 & October \\
\hline
\end{tabular}




\begin{tabular}{|c|c|c|c|c|c|c|c|}
\hline $\begin{array}{l}\text { Depth - Min } \\
(\mathrm{m})\end{array}$ & $\begin{array}{c}\text { Depth-Max } \\
(\mathrm{m})\end{array}$ & Habitats & Abr & Species & $\begin{array}{l}\text { Diameter } \\
(\mathrm{cm})\end{array}$ & Year & Month \\
\hline 95 & 96 & Sand & Ecsep & Echinaster sepositus & 6 & 2015 & October \\
\hline 95 & 96 & Sand & Ecsep & Echinaster sepositus & 6 & 2015 & October \\
\hline 95 & 96 & Sand & Ecsep & Echinaster sepositus & 6 & 2015 & October \\
\hline 95 & 96 & Sand & Ecsep & Echinaster sepositus & 6 & 2015 & October \\
\hline 180 & 210 & Sand & Ecsep & Echinaster sepositus & 6 & 2015 & December \\
\hline 180 & 210 & Sand & Ecsep & Echinaster sepositus & 6 & 2015 & December \\
\hline 180 & 210 & Sand & Ecsep & Echinaster sepositus & 8 & 2015 & December \\
\hline 120 & 145 & Mud & Ecsep & Echinaster sepositus & 8 & 2015 & December \\
\hline 120 & 145 & Mud & Ecsep & Echinaster sepositus & 8 & 2015 & December \\
\hline 120 & 145 & Mud & Ecsep & Echinaster sepositus & 8 & 2015 & December \\
\hline 120 & 145 & Mud & Ecsep & Echinaster sepositus & 9 & 2015 & December \\
\hline 68 & 77 & Sand & Ecsep & Echinaster sepositus & 9 & 2015 & December \\
\hline 68 & 77 & Sand & Ecsep & Echinaster sepositus & 9 & 2015 & December \\
\hline 68 & 77 & Sand & Ecsep & Echinaster sepositus & 9 & 2015 & December \\
\hline 68 & 77 & Sand & Ecsep & Echinaster sepositus & 9 & 2015 & December \\
\hline 67 & 70 & Sand & Ecsep & Echinaster sepositus & 10 & 2015 & December \\
\hline 67 & 70 & Sand & Ecsep & Echinaster sepositus & 10 & 2015 & December \\
\hline 67 & 70 & Sand & Ecsep & Echinaster sepositus & 10 & 2015 & December \\
\hline 67 & 70 & Sand & Ecsep & Echinaster sepositus & 10 & 2015 & December \\
\hline 67 & 70 & Sand & Ecsep & Echinaster sepositus & 10 & 2015 & December \\
\hline 80 & 111 & Mud & Ecsep & Echinaster sepositus & 11 & 2015 & December \\
\hline 80 & 111 & Mud & Ecsep & Echinaster sepositus & 11 & 2015 & December \\
\hline 90 & 105 & Mud-Sand & Ecsep & Echinaster sepositus & 11 & 2015 & December \\
\hline 90 & 105 & Mud-Sand & Ecsep & Echinaster sepositus & 11 & 2015 & December \\
\hline 90 & 105 & Mud-Sand & Ecsep & Echinaster sepositus & 11 & 2015 & December \\
\hline 80 & 120 & Sand & Ecsep & Echinaster sepositus & 11 & 2015 & December \\
\hline 80 & 120 & Sand & Ecsep & Echinaster sepositus & 11 & 2015 & December \\
\hline 80 & 120 & Sand & Ecsep & Echinaster sepositus & 12 & 2015 & December \\
\hline 100 & 115 & Sand & Ecsep & Echinaster sepositus & 12 & 2016 & March \\
\hline 100 & 115 & Sand & Ecsep & Echinaster sepositus & 12 & 2016 & March \\
\hline 100 & 115 & Sand & Ecsep & Echinaster sepositus & 12 & 2016 & March \\
\hline 100 & 115 & Sand & Ecsep & Echinaster sepositus & 12 & 2016 & March \\
\hline 50 & 65 & Sand & Ecsep & Echinaster sepositus & 12 & 2016 & March \\
\hline 50 & 65 & Sand & Ecsep & Echinaster sepositus & 12 & 2016 & March \\
\hline 50 & 65 & Sand & Ecsep & Echinaster sepositus & 12 & 2016 & March \\
\hline 50 & 60 & Rock & Ecsep & Echinaster sepositus & 12 & 2016 & August \\
\hline 50 & 60 & Rock & Ecsep & Echinaster sepositus & 13 & 2016 & August \\
\hline 50 & 60 & Rock & Ecsep & Echinaster sepositus & 13 & 2016 & August \\
\hline 50 & 60 & Rock & Ecsep & Echinaster sepositus & 13 & 2016 & August \\
\hline 101 & 115 & Sand & Ecsep & Echinaster sepositus & 13 & 2016 & August \\
\hline 101 & 115 & Sand & Ecsep & Echinaster sepositus & 13 & 2016 & August \\
\hline 101 & 115 & Sand & Ecsep & Echinaster sepositus & 13 & 2016 & August \\
\hline 101 & 115 & Sand & Ecsep & Echinaster sepositus & 13 & 2016 & August \\
\hline 101 & 115 & Sand & Ecsep & Echinaster sepositus & 13 & 2016 & August \\
\hline 104 & 105 & Sand & Ecsep & Echinaster sepositus & 14 & 2016 & August \\
\hline 104 & 105 & Sand & Ecsep & Echinaster sepositus & 14 & 2016 & August \\
\hline 85 & 100 & Rock & Ecsep & Echinaster sepositus & 14 & 2016 & April \\
\hline 85 & 100 & Rock & Ecsep & Echinaster sepositus & 14 & 2016 & April \\
\hline 185 & 220 & Sand & Ecsep & Echinaster sepositus & 14 & 2016 & April \\
\hline 185 & 220 & Sand & Ecsep & Echinaster sepositus & 15 & 2016 & April \\
\hline 185 & 220 & Sand & Ecsep & Echinaster sepositus & 15 & 2016 & April \\
\hline 185 & 220 & Sand & Ecsep & Echinaster sepositus & 15 & 2016 & April \\
\hline 82 & 105 & Rock & Ecsep & Echinaster sepositus & 15 & 2016 & April \\
\hline 82 & 105 & Rock & Ecsep & Echinaster sepositus & 15 & 2016 & April \\
\hline 82 & 105 & Rock & Ecsep & Echinaster sepositus & 15 & 2016 & April \\
\hline 51 & 58 & Mud & Ecsep & Echinaster sepositus & 15 & 2016 & June \\
\hline 51 & 58 & Mud & Ecsep & Echinaster sepositus & 15 & 2016 & June \\
\hline 130 & 140 & Sand & Ecsep & Echinaster sepositus & 15 & 2016 & June \\
\hline 130 & 140 & Sand & Ecsep & Echinaster sepositus & 15 & 2016 & June \\
\hline 79 & 110 & Sand & Ecsep & Echinaster sepositus & 15 & 2016 & June \\
\hline 79 & 110 & Sand & Ecsep & Echinaster sepositus & 16 & 2016 & June \\
\hline 79 & 110 & Sand & Ecsep & Echinaster sepositus & 16 & 2016 & June \\
\hline 81 & 100 & Sand & Ecsep & Echinaster sepositus & 16 & 2016 & June \\
\hline 81 & 100 & Sand & Ecsep & Echinaster sepositus & 16 & 2016 & June \\
\hline 90 & 160 & Mud-Sand & Ecsep & Echinaster sepositus & 16 & 2016 & September \\
\hline 90 & 160 & Mud-Sand & Ecsep & Echinaster sepositus & 16 & 2016 & September \\
\hline 78 & 84 & Rock & Ecsep & Echinaster sepositus & 16 & 2016 & September \\
\hline 135 & 170 & Sand & Ecsep & Echinaster sepositus & 16 & 2016 & September \\
\hline 135 & 170 & Sand & Ecsep & Echinaster sepositus & 16 & 2016 & September \\
\hline 135 & 170 & Sand & Ecsep & Echinaster sepositus & 16 & 2016 & September \\
\hline 158 & 164 & Sand & Ecsep & Echinaster sepositus & 8 & 2016 & July \\
\hline 70 & 85 & Rock & Ecsep & Echinaster sepositus & 16 & 2016 & July \\
\hline 70 & 85 & Rock & Ecsep & Echinaster sepositus & 16 & 2016 & July \\
\hline 70 & 85 & Rock & Ecsep & Echinaster sepositus & 16 & 2016 & July \\
\hline 70 & 85 & Rock & Ecsep & Echinaster sepositus & 16 & 2016 & July \\
\hline 70 & 85 & Rock & Ecsep & Echinaster sepositus & 16 & 2016 & July \\
\hline 70 & 85 & Rock & Ecsep & Echinaster sepositus & 16 & 2016 & July \\
\hline
\end{tabular}




\begin{tabular}{|c|c|c|c|c|c|c|c|}
\hline $\begin{array}{c}\text { Depth - Min } \\
(\mathrm{m})\end{array}$ & $\begin{array}{c}\text { Depth-Max } \\
(\mathrm{m})\end{array}$ & Habitats & Abr & Species & $\begin{array}{l}\text { Diameter } \\
(\mathrm{cm})\end{array}$ & Year & Month \\
\hline 70 & 85 & Sand & Ecsep & Echinaster sepositus & 16 & 2016 & July \\
\hline 3 & 5 & Rock & Ecsep & Echinaster sepositus & 12 & 2016 & July \\
\hline 3 & 5 & Rock & Ecsep & Echinaster sepositus & 13 & 2016 & July \\
\hline 70 & 85 & Rock & Haatt & Hacilia attenuata & 8 & 2016 & July \\
\hline 68 & 77 & Sand & Ecsep & Echinaster sepositus & 13 & 2015 & December \\
\hline 68 & 77 & Sand & Ecsep & Echinaster sepositus & 13 & 2015 & December \\
\hline 90 & 105 & Mud-Sand & Ecsep & Echinaster sepositus & 7 & 2015 & December \\
\hline 90 & 105 & Mud-Sand & Ecsep & Echinaster sepositus & 7 & 2015 & December \\
\hline 100 & 115 & Sand & Ecsep & Echinaster sepositus & 8 & 2016 & March \\
\hline 101 & 115 & Sand & Ecsep & Echinaster sepositus & 8 & 2016 & August \\
\hline 104 & 105 & Sand & Ecsep & Echinaster sepositus & 8 & 2016 & August \\
\hline 104 & 105 & Sand & Ecsep & Echinaster sepositus & 8 & 2016 & August \\
\hline 70 & 95 & Rock & Ecsep & Echinaster sepositus & 8 & 2016 & June \\
\hline 70 & 95 & Rock & Ecsep & Echinaster sepositus & 8.5 & 2016 & June \\
\hline 72 & 90 & Rock & Ecsep & Echinaster sepositus & 8.5 & 2016 & June \\
\hline 72 & 90 & Rock & Ecsep & Echinaster sepositus & 8.5 & 2016 & June \\
\hline 160 & 161 & Sand & Ecsep & Echinaster sepositus & 8.5 & 2016 & July \\
\hline 160 & 161 & Sand & Ecsep & Echinaster sepositus & 8.5 & 2015 & July \\
\hline 91 & 95 & Sand & Luatl & Luidia atlantidea & 6 & 2015 & October \\
\hline 175 & 193 & Sand & Lusa & Luidia sarssi & 8 & 2016 & July \\
\hline 180 & 210 & Sand & Magla & Marthasterias glacialis & 14 & 2015 & October \\
\hline 180 & 210 & Sand & Magla & Marthasterias glacialis & 14 & 2015 & December \\
\hline 120 & 145 & Mud & Magla & Marthasterias glacialis & 15 & 2015 & December \\
\hline 80 & 111 & Mud & Magla & Marthasterias glacialis & 15 & 2015 & December \\
\hline 80 & 113 & Mud & Magla & Marthasterias glacialis & 15 & 2016 & March \\
\hline 90 & 120 & Sand & Magla & Marthasterias glacialis & 16 & 2016 & March \\
\hline 70 & 100 & Sand & Magla & Marthasterias glacialis & 16 & 2016 & March \\
\hline 70 & 100 & Sand & Magla & Marthasterias glacialis & 16 & 2016 & March \\
\hline 75 & 101 & Rock & Magla & Marthasterias glacialis & 16 & 2016 & August \\
\hline 78 & 100 & Mud-Sand & Magla & Marthasterias glacialis & 18 & 2016 & June \\
\hline 180 & 200 & Sand & Magla & Marthasterias glacialis & 18 & 2016 & June \\
\hline 85 & 98 & Mud & Tesub & Tethyaster subinermis & 4 & 2015 & October \\
\hline 98 & 105 & Sand & Tesub & Tethyaster subinermis & 4 & 2015 & October \\
\hline 98 & 105 & Sand & Tesub & Tethyaster subinermis & 4 & 2015 & October \\
\hline 98 & 105 & Sand & Tesub & Tethyaster subinermis & 4 & 2015 & October \\
\hline 90 & 105 & Mud-Sand & Tesub & Tethyaster subinermis & 4 & 2015 & December \\
\hline 90 & 105 & Mud-Sand & Tesub & Tethyaster subinermis & 4 & 2015 & December \\
\hline 90 & 110 & Mud-Sand & Tesub & Tethyaster subinermis & 5 & 2015 & December \\
\hline 90 & 110 & Mud-Sand & Tesub & Tethyaster subinermis & 5 & 2015 & December \\
\hline 79 & 80 & Sand & Tesub & Tethyaster subinermis & 5 & 2016 & March \\
\hline 79 & 80 & Sand & Tesub & Tethyaster subinermis & 5 & 2016 & March \\
\hline 79 & 80 & Sand & Tesub & Tethyaster subinermis & 5 & 2016 & March \\
\hline 100 & 125 & Sand & Tesub & Tethyaster subinermis & 6 & 2016 & March \\
\hline 100 & 125 & Sand & Tesub & Tethyaster subinermis & 6 & 2016 & March \\
\hline 50 & 60 & Sand & Tesub & Tethyaster subinermis & 6 & 2016 & March \\
\hline 50 & 60 & Sand & Tesub & Tethyaster subinermis & 6 & 2016 & March \\
\hline 101 & 115 & Sand & Tesub & Tethyaster subinermis & 6 & 2016 & August \\
\hline 104 & 105 & Sand & Tesub & Tethyaster subinermis & 6 & 2016 & August \\
\hline 104 & 105 & Sand & Tesub & Tethyaster subinermis & 6 & 2016 & August \\
\hline 85 & 100 & Rock & Tesub & Tethyaster subinermis & 7 & 2016 & April \\
\hline 185 & 220 & Sand & Tesub & Tethyaster subinermis & 7 & 2016 & April \\
\hline 185 & 220 & Sand & Tesub & Tethyaster subinermis & 7 & 2016 & April \\
\hline 152 & 177 & Sand & Tesub & Tethyaster subinermis & 7 & 2016 & April \\
\hline 51 & 58 & Mud & Tesub & Tethyaster subinermis & 7 & 2016 & June \\
\hline 51 & 58 & Mud & Tesub & Tethyaster subinermis & 7 & 2016 & June \\
\hline 81 & 100 & Sand & Tesub & Tethyaster subinermis & 8 & 2016 & June \\
\hline 85 & 100 & Sand & Tesub & Tethyaster subinermis & 8 & 2016 & September \\
\hline 85 & 100 & Sand & Tesub & Tethyaster subinermis & 8 & 2016 & September \\
\hline 85 & 100 & Sand & Tesub & Tethyaster subinermis & 8 & 2016 & September \\
\hline 90 & 160 & Mud-Sand & Tesub & Tethyaster subinermis & 8 & 2016 & September \\
\hline 135 & 170 & Sand & Tesub & Tethyaster subinermis & 8 & 2016 & September \\
\hline 135 & 170 & Sand & Tesub & Tethyaster subinermis & 8 & 2016 & September \\
\hline 160 & 194 & Mud & Tesub & Tethyaster subinermis & 12 & 2016 & July \\
\hline 180 & 192 & Mud & Tesub & Tethyaster subinermis & 12 & 2016 & July \\
\hline 175 & 193 & Sand & Tesub & Tethyaster subinermis & 12 & 2016 & July \\
\hline 175 & 193 & Sand & Tesub & Tethyaster subinermis & 12 & 2016 & July \\
\hline 176 & 179 & Mud-Sand & Tesub & Tethyaster subinermis & 12 & 2016 & July \\
\hline 181 & 186 & Mud-Sand & Tesub & Tethyaster subinermis & 15 & 2016 & July \\
\hline 181 & 190 & Sand & Tesub & Tethyaster subinermis & 15 & 2016 & July \\
\hline 181 & 190 & Sand & Tesub & Tethyaster subinermis & 15 & 2016 & July \\
\hline 85 & 98 & Mud & Anbif & Antedon bifida & 4 & 2015 & October \\
\hline 85 & 98 & Mud & Anbif & Antedon bifida & 4 & 2015 & October \\
\hline 85 & 98 & Mud & Anbif & Antedon bifida & 4 & 2015 & October \\
\hline 98 & 105 & Sand & Anbif & Antedon bifida & 4 & 2015 & October \\
\hline 98 & 105 & Sand & Anbif & Antedon bifida & 4 & 2015 & October \\
\hline 98 & 105 & Sand & Anbif & Antedon bifida & 4 & 2015 & October \\
\hline 68 & 94 & Mud-Sand & Anbif & Antedon bifida & 4 & 2015 & October \\
\hline
\end{tabular}




\begin{tabular}{|c|c|c|c|c|c|c|c|}
\hline $\begin{array}{l}\text { Depth - Min } \\
(\mathrm{m})\end{array}$ & $\begin{array}{c}\text { Depth-Max } \\
\text { (m) }\end{array}$ & Habitats & Abr & Species & $\begin{array}{l}\text { Diameter } \\
(\mathrm{cm})\end{array}$ & Year & Month \\
\hline 68 & 94 & Mud-Sand & Anbif & Antedon bifida & 4 & 2015 & October \\
\hline 91 & 95 & Sand & Anbif & Antedon bifida & 4 & 2015 & October \\
\hline 91 & 95 & Sand & Anbif & Antedon bifida & 4 & 2015 & October \\
\hline 91 & 95 & Sand & Anbif & Antedon bifida & 4.5 & 2015 & October \\
\hline 91 & 95 & Sand & Anbif & Antedon bifida & 4.5 & 2015 & October \\
\hline 166 & 190 & Sand & Anbif & Antedon bifida & 4.5 & 2015 & December \\
\hline 166 & 190 & Sand & Anbif & Antedon bifida & 4.5 & 2015 & December \\
\hline 68 & 77 & Sand & Anbif & Antedon bifida & 4.5 & 2015 & December \\
\hline 68 & 77 & Sand & Anbif & Antedon bifida & 4.5 & 2015 & December \\
\hline 67 & 70 & Sand & Anbif & Antedon bifida & 4.5 & 2015 & December \\
\hline 67 & 70 & Sand & Anbif & Antedon bifida & 5 & 2015 & December \\
\hline 115 & 130 & Sand & Anbif & Antedon bifida & 5 & 2015 & December \\
\hline 115 & 130 & Sand & Anbif & Antedon bifida & 5 & 2015 & December \\
\hline 115 & 130 & Sand & Anbif & Antedon bifida & 5 & 2015 & December \\
\hline 115 & 130 & Sand & Anbif & Antedon bifida & 5 & 2015 & December \\
\hline 90 & 110 & Mud-Sand & Anbif & Antedon bifida & 5 & 2015 & December \\
\hline 90 & 110 & Mud-Sand & Anbif & Antedon bifida & 5 & 2015 & December \\
\hline 80 & 120 & Sand & Anbif & Antedon bifida & 5 & 2015 & December \\
\hline 80 & 120 & Sand & Anbif & Antedon bifida & 5 & 2015 & December \\
\hline 80 & 120 & Sand & Anbif & Antedon bifida & 5 & 2015 & December \\
\hline 79 & 80 & Sand & Anbif & Antedon bifida & 6 & 2016 & March \\
\hline 79 & 80 & Sand & Anbif & Antedon bifida & 6 & 2016 & March \\
\hline 80 & 113 & Mud & Anbif & Antedon bifida & 6 & 2016 & March \\
\hline 80 & 113 & Mud & Anbif & Antedon bifida & 6 & 2016 & March \\
\hline 80 & 113 & Mud & Anbif & Antedon bifida & 6 & 2016 & March \\
\hline 90 & 120 & Sand & Anbif & Antedon bifida & 6 & 2016 & March \\
\hline 90 & 120 & Sand & Anbif & Antedon bifida & 6 & 2016 & March \\
\hline 70 & 100 & Sand & Anbif & Antedon bifida & 6 & 2016 & March \\
\hline 70 & 100 & Sand & Anbif & Antedon bifida & 6 & 2016 & March \\
\hline 70 & 125 & Sand & Anbif & Antedon bifida & 6 & 2016 & March \\
\hline 70 & 125 & Sand & Anbif & Antedon bifida & 7 & 2016 & March \\
\hline 50 & 60 & Rock & Anbif & Antedon bifida & 7 & 2016 & March \\
\hline 50 & 60 & Rock & Anbif & Antedon bifida & 7 & 2016 & March \\
\hline 50 & 60 & Rock & Anbif & Antedon bifida & 7 & 2016 & August \\
\hline 50 & 60 & Rock & Anbif & Antedon bifida & 7 & 2016 & August \\
\hline 50 & 103 & Sand & Anbif & Antedon bifida & 7 & 2016 & August \\
\hline 50 & 103 & Sand & Anbif & Antedon bifida & 7 & 2016 & August \\
\hline 77 & 96 & Mud & Anbif & Antedon bifida & 7.5 & 2016 & August \\
\hline 77 & 96 & Mud & Anbif & Antedon bifida & 7.5 & 2016 & August \\
\hline 101 & 115 & Sand & Anbif & Antedon bifida & 8 & 2016 & August \\
\hline 101 & 115 & Sand & Anbif & Antedon bifida & 8 & 2016 & August \\
\hline 104 & 105 & Sand & Anbif & Antedon bifida & 8 & 2016 & August \\
\hline 104 & 105 & Sand & Anbif & Antedon bifida & 8 & 2016 & August \\
\hline 85 & 98 & Mud & Anmed & Antedon mediterranea & 4 & 2015 & October \\
\hline 85 & 98 & Mud & Anmed & Antedon mediterranea & 4 & 2015 & October \\
\hline 85 & 98 & Mud & Anmed & Antedon mediterranea & 4 & 2015 & October \\
\hline 98 & 105 & Sand & Anmed & Antedon mediterranea & 4 & 2015 & October \\
\hline 98 & 105 & Sand & Anmed & Antedon mediterranea & 4 & 2015 & October \\
\hline 98 & 105 & Sand & Anmed & Antedon mediterranea & 4 & 2015 & October \\
\hline 68 & 94 & Mud-Sand & Anmed & Antedon mediterranea & 4 & 2015 & October \\
\hline 68 & 94 & Mud-Sand & Anmed & Antedon mediterranea & 4 & 2015 & October \\
\hline 91 & 95 & Sand & Anmed & Antedon mediterranea & 4 & 2015 & October \\
\hline 91 & 95 & Sand & Anmed & Antedon mediterranea & 4 & 2015 & October \\
\hline 91 & 95 & Sand & Anmed & Antedon mediterranea & 4.5 & 2015 & October \\
\hline 91 & 95 & Sand & Anmed & Antedon mediterranea & 4.5 & 2015 & October \\
\hline 166 & 199 & Sand & Anmed & Antedon mediterranea & 4.5 & 2015 & December \\
\hline 166 & 199 & Sand & Anmed & Antedon mediterranea & 4.5 & 2015 & December \\
\hline 68 & 77 & Sand & Anmed & Antedon mediterranea & 4.5 & 2015 & December \\
\hline 68 & 77 & Sand & Anmed & Antedon mediterranea & 4.5 & 2015 & December \\
\hline 67 & 70 & Sand & Anmed & Antedon mediterranea & 4.5 & 2015 & December \\
\hline 67 & 70 & Sand & Anmed & Antedon mediterranea & 5 & 2015 & December \\
\hline 115 & 130 & Sand & Anmed & Antedon mediterranea & 5 & 2015 & December \\
\hline 115 & 130 & Sand & Anmed & Antedon mediterranea & 5 & 2015 & December \\
\hline 115 & 130 & Sand & Anmed & Antedon mediterranea & 5 & 2015 & December \\
\hline 115 & 130 & Sand & Anmed & Antedon mediterranea & 5 & 2015 & December \\
\hline 90 & 110 & Mud-Sand & Anmed & Antedon mediterranea & 5 & 2015 & December \\
\hline 90 & 110 & Mud-Sand & Anmed & Antedon mediterranea & 5 & 2015 & December \\
\hline 80 & 120 & Sand & Anmed & Antedon mediterranea & 5 & 2015 & December \\
\hline 80 & 120 & Sand & Anmed & Antedon mediterranea & 5 & 2015 & December \\
\hline 80 & 120 & Sand & Anmed & Antedon mediterranea & 5 & 2015 & December \\
\hline 79 & 80 & Sand & Anmed & Antedon mediterranea & 5 & 2016 & March \\
\hline 79 & 80 & Sand & Anmed & Antedon mediterranea & 5 & 2016 & March \\
\hline 80 & 113 & Mud & Anmed & Antedon mediterranea & 5 & 2016 & March \\
\hline 80 & 113 & Mud & Anmed & Antedon mediterranea & 5 & 2016 & March \\
\hline 80 & 113 & Mud & Anmed & Antedon mediterranea & 5 & 2016 & March \\
\hline 90 & 120 & Sand & Anmed & Antedon mediterranea & 5 & 2016 & March \\
\hline 90 & 120 & Sand & Anmed & Antedon mediterranea & 6 & 2016 & March \\
\hline
\end{tabular}




\begin{tabular}{|c|c|c|c|c|c|c|c|}
\hline $\begin{array}{l}\text { Depth - Min } \\
(\mathrm{m})\end{array}$ & $\begin{array}{c}\text { Depth-Max } \\
(\mathrm{m})\end{array}$ & Habitats & Abr & Species & $\begin{array}{l}\text { Diameter } \\
\quad(\mathrm{cm})\end{array}$ & Year & Month \\
\hline 70 & 100 & Sand & Anmed & Antedon mediterranea & 6 & 2016 & March \\
\hline 70 & 100 & Sand & Anmed & Antedon mediterranea & 6 & 2016 & March \\
\hline 100 & 125 & Sand & Anmed & Antedon mediterranea & 6 & 2016 & March \\
\hline 100 & 125 & Sand & Anmed & Antedon mediterranea & 6 & 2016 & March \\
\hline 50 & 60 & Rock & Anmed & Antedon mediterranea & 6 & 2016 & March \\
\hline 50 & 60 & Rock & Anmed & Antedon mediterranea & 6 & 2016 & March \\
\hline 50 & 60 & Rock & Anmed & Antedon mediterranea & 6 & 2016 & August \\
\hline 50 & 60 & Rock & Anmed & Antedon mediterranea & 6 & 2016 & August \\
\hline 50 & 103 & Sand & Anmed & Antedon mediterranea & 7 & 2016 & August \\
\hline 50 & 103 & Sand & Anmed & Antedon mediterranea & 7 & 2016 & August \\
\hline 77 & 96 & Mud & Anmed & Antedon mediterranea & 7 & 2016 & August \\
\hline 77 & 96 & Mud & Anmed & Antedon mediterranea & 7 & 2016 & August \\
\hline 101 & 115 & Sand & Anmed & Antedon mediterranea & 7 & 2016 & August \\
\hline 101 & 115 & Sand & Anmed & Antedon mediterranea & 7 & 2016 & August \\
\hline 104 & 105 & Sand & Anmed & Antedon mediterranea & 7 & 2016 & August \\
\hline 104 & 105 & Sand & Anmed & Antedon mediterranea & 7 & 2016 & August \\
\hline 85 & 100 & Rock & Anmed & Antedon mediterranea & 7 & 2016 & April \\
\hline 85 & 100 & Rock & Anmed & Antedon mediterranea & 7 & 2016 & April \\
\hline 152 & 177 & Sand & Anmed & Antedon mediterranea & 7 & 2016 & April \\
\hline 152 & 177 & Sand & Anmed & Antedon mediterranea & 7 & 2016 & April \\
\hline 152 & 177 & Sand & Anmed & Antedon mediterranea & 7 & 2016 & April \\
\hline 152 & 177 & Sand & Anmed & Antedon mediterranea & 7 & 2016 & April \\
\hline 78 & 100 & Mud-Sand & Anmed & Antedon mediterranea & 7 & 2016 & June \\
\hline 78 & 100 & Mud-Sand & Anmed & Antedon mediterranea & 7 & 2016 & June \\
\hline 51 & 58 & Mud & Anmed & Antedon mediterranea & 7 & 2016 & June \\
\hline 70 & 95 & Rock & Anmed & Antedon mediterranea & 7 & 2016 & June \\
\hline 70 & 95 & Rock & Anmed & Antedon mediterranea & 7 & 2016 & June \\
\hline 70 & 95 & Rock & Anmed & Antedon mediterranea & 7 & 2016 & June \\
\hline 79 & 110 & Sand & Anmed & Antedon mediterranea & 7 & 2016 & June \\
\hline 79 & 110 & Sand & Anmed & Antedon mediterranea & 7 & 2016 & June \\
\hline 81 & 100 & Sand & Anmed & Antedon mediterranea & 7 & 2016 & June \\
\hline 81 & 100 & Sand & Anmed & Antedon mediterranea & 7 & 2016 & June \\
\hline 85 & 100 & Sand & Anmed & Antedon mediterranea & 7 & 2016 & September \\
\hline 85 & 100 & Sand & Anmed & Antedon mediterranea & 7 & 2016 & September \\
\hline 72 & 90 & Rock & Lepha & Leptometra phalangium & 4.5 & 2016 & September \\
\hline 72 & 90 & Rock & Lepha & Leptometra phalangium & 4.5 & 2016 & September \\
\hline 78 & 84 & Rock & Lepha & Leptometra phalangium & 4.5 & 2016 & September \\
\hline 78 & 84 & Rock & Lepha & Leptometra phalangium & 4.5 & 2016 & September \\
\hline 135 & 170 & Sand & Lepha & Leptometra phalangium & 4.5 & 2016 & September \\
\hline 135 & 170 & Sand & Lepha & Leptometra phalangium & 4.5 & 2016 & September \\
\hline 135 & 170 & Sand & Lepha & Leptometra phalangium & 5 & 2016 & September \\
\hline 160 & 194 & Mud & Lepha & Leptometra phalangium & 5 & 2016 & July \\
\hline 160 & 194 & Mud & Lepha & Leptometra phalangium & 5 & 2016 & July \\
\hline 160 & 194 & Mud & Lepha & Leptometra phalangium & 5 & 2016 & July \\
\hline 160 & 194 & Mud & Lepha & Leptometra phalangium & 5 & 2016 & July \\
\hline 180 & 192 & Mud & Lepha & Leptometra phalangium & 5 & 2016 & July \\
\hline 180 & 192 & Mud & Lepha & Leptometra phalangium & 5 & 2016 & July \\
\hline 180 & 192 & Mud & Lepha & Leptometra phalangium & 5 & 2016 & July \\
\hline 175 & 193 & Sand & Lepha & Leptometra phalangium & 5 & 2016 & July \\
\hline 175 & 193 & Sand & Lepha & Leptometra phalangium & 5 & 2016 & July \\
\hline 175 & 193 & Sand & Lepha & Leptometra phalangium & 5 & 2016 & July \\
\hline 176 & 179 & Mud-Sand & Lepha & Leptometra phalangium & 5 & 2016 & July \\
\hline 176 & 179 & Mud-Sand & Lepha & Leptometra phalangium & 5 & 2016 & July \\
\hline 176 & 179 & Mud-Sand & Lepha & Leptometra phalangium & 5 & 2016 & July \\
\hline 183 & 193 & Sand & Lepha & Leptometra phalangium & 5 & 2016 & July \\
\hline 183 & 193 & Sand & Lepha & Leptometra phalangium & 5.5 & 2016 & July \\
\hline 142 & 163 & Mud & Lepha & Leptometra phalangium & 5.5 & 2016 & July \\
\hline 142 & 163 & Mud & Lepha & Leptometra phalangium & 5.5 & 2016 & July \\
\hline 142 & 163 & Mud & Lepha & Leptometra phalangium & 5.5 & 2016 & July \\
\hline 181 & 186 & Mud-Sand & Lepha & Leptometra phalangium & 5.5 & 2016 & July \\
\hline 181 & 186 & Mud-Sand & Lepha & Leptometra phalangium & 5.5 & 2016 & July \\
\hline 179 & 185 & Sand & Lepha & Leptometra phalangium & 5.5 & 2016 & July \\
\hline 179 & 185 & Sand & Lepha & Leptometra phalangium & 5.5 & 2016 & July \\
\hline 179 & 185 & Sand & Lepha & Leptometra phalangium & 5.5 & 2016 & July \\
\hline 181 & 190 & Sand & Lepha & Leptometra phalangium & 5.5 & 2016 & July \\
\hline 181 & 190 & Sand & Lepha & Leptometra phalangium & 5.5 & 2016 & July \\
\hline 181 & 190 & Sand & Lepha & Leptometra phalangium & 5.5 & 2016 & July \\
\hline 0.2 & 0.6 & Algea & Arlix & Arbacia lixula & 4.5 & 2016 & July \\
\hline 0.2 & 0.6 & Algea & Arlix & Arbacia lixula & 4.5 & 2016 & July \\
\hline 0.2 & 0.6 & Algea & Arlix & Arbacia lixula & 5 & 2016 & July \\
\hline 0.2 & 0.6 & Algea & Arlix & Arbacia lixula & 5 & 2016 & July \\
\hline 3 & 6 & Sand & Arlix & Arbacia lixula & 5 & 2016 & May \\
\hline 3 & 6 & Sand & Arlix & Arbacia lixula & 5 & 2016 & May \\
\hline 3 & 6 & Sand & Arlix & Arbacia lixula & 5 & 2016 & May \\
\hline 3 & 6 & Sand & Arlix & Arbacia lixula & 5.5 & 2016 & May \\
\hline 3 & 5 & Sand & Arlix & Arbacia lixula & 5.5 & 2016 & July \\
\hline 0 & 3 & Sand & Arlix & Arbacia lixula & 6 & 2016 & July \\
\hline
\end{tabular}




\begin{tabular}{|c|c|c|c|c|c|c|c|}
\hline $\begin{array}{l}\text { Depth - Min } \\
\text { (m) }\end{array}$ & $\begin{array}{c}\text { Depth-Max } \\
(\mathrm{m})\end{array}$ & Habitats & Abr & Species & $\begin{array}{l}\text { Diameter } \\
(\mathrm{cm})\end{array}$ & Year & Month \\
\hline 0 & 3 & Rock & Arlix & Arbacia lixula & 6 & 2016 & July \\
\hline 3 & 5 & Rock & Arlix & Arbacia lixula & 6 & 2016 & July \\
\hline 85 & 98 & Mud & Celon & Centrostephanus longispinus & 5 & 2015 & October \\
\hline 85 & 98 & Mud & Celon & Centrostephanus longispinus & 5 & 2015 & October \\
\hline 85 & 98 & Mud & Celon & Centrostephanus longispinus & 5 & 2015 & October \\
\hline 98 & 105 & Sand & Celon & Centrostephanus longispinus & 5 & 2015 & October \\
\hline 98 & 105 & Sand & Celon & Centrostephanus longispinus & 5 & 2015 & October \\
\hline 98 & 105 & Sand & Celon & Centrostephanus longispinus & 5 & 2015 & October \\
\hline 98 & 105 & Sand & Celon & Centrostephanus longispinus & 5 & 2015 & October \\
\hline 68 & 94 & Mud-Sand & Celon & Centrostephanus longispinus & 6 & 2015 & October \\
\hline 68 & 94 & Mud-Sand & Celon & Centrostephanus longispinus & 6 & 2015 & October \\
\hline 91 & 95 & Sand & Celon & Centrostephanus longispinus & 6 & 2015 & October \\
\hline 91 & 95 & Sand & Celon & Centrostephanus longispinus & 6 & 2015 & October \\
\hline 91 & 95 & Sand & Celon & Centrostephanus longispinus & 6 & 2015 & October \\
\hline 91 & 95 & Sand & Celon & Centrostephanus longispinus & 6 & 2015 & October \\
\hline 91 & 95 & Sand & Celon & Centrostephanus longispinus & 6 & 2015 & October \\
\hline 180 & 210 & Sand & Celon & Centrostephanus longispinus & 6 & 2015 & December \\
\hline 180 & 210 & Sand & Celon & Centrostephanus longispinus & 6 & 2015 & December \\
\hline 180 & 210 & Sand & Celon & Centrostephanus longispinus & 6 & 2015 & December \\
\hline 120 & 145 & Mud & Celon & Centrostephanus longispinus & 6 & 2015 & December \\
\hline 120 & 145 & Mud & Celon & Centrostephanus longispinus & 6 & 2015 & December \\
\hline 68 & 77 & Sand & Celon & Centrostephanus longispinus & 6 & 2015 & December \\
\hline 68 & 77 & Sand & Celon & Centrostephanus longispinus & 6 & 2015 & December \\
\hline 67 & 70 & Sand & Celon & Centrostephanus longispinus & 7 & 2015 & December \\
\hline 67 & 70 & Sand & Celon & Centrostephanus longispinus & 7 & 2015 & December \\
\hline 80 & 111 & Mud & Celon & Centrostephanus longispinus & 7 & 2015 & December \\
\hline 80 & 111 & Mud & Celon & Centrostephanus longispinus & 7 & 2015 & December \\
\hline 80 & 111 & Mud & Celon & Centrostephanus longispinus & 7 & 2015 & December \\
\hline 90 & 105 & Mud-Sand & Celon & Centrostephanus longispinus & 7 & 2015 & December \\
\hline 90 & 105 & Mud-Sand & Celon & Centrostephanus longispinus & 7 & 2015 & December \\
\hline 79 & 80 & Sand & Celon & Centrostephanus longispinus & 7 & 2016 & March \\
\hline 79 & 80 & Sand & Celon & Centrostephanus longispinus & 7 & 2016 & March \\
\hline 79 & 80 & Sand & Celon & Centrostephanus longispinus & 7 & 2016 & March \\
\hline 80 & 113 & Mud & Celon & Centrostephanus longispinus & 8 & 2016 & March \\
\hline 80 & 113 & Mud & Celon & Centrostephanus longispinus & 8 & 2016 & March \\
\hline 80 & 113 & Mud & Celon & Centrostephanus longispinus & 8 & 2016 & March \\
\hline 100 & 115 & Sand & Celon & Centrostephanus longispinus & 8 & 2016 & March \\
\hline 100 & 115 & Sand & Celon & Centrostephanus longispinus & 8 & 2016 & March \\
\hline 50 & 60 & Rock & Celon & Centrostephanus longispinus & 8 & 2016 & March \\
\hline 50 & 60 & Rock & Celon & Centrostephanus longispinus & 8 & 2016 & March \\
\hline 50 & 60 & Rock & Celon & Centrostephanus longispinus & 8 & 2016 & March \\
\hline 50 & 60 & Rock & Celon & Centrostephanus longispinus & 8 & 2016 & August \\
\hline 50 & 60 & Rock & Celon & Centrostephanus longispinus & 8 & 2016 & August \\
\hline 50 & 60 & Rock & Celon & Centrostephanus longispinus & 9 & 2016 & August \\
\hline 75 & 101 & Rock & Celon & Centrostephanus longispinus & 9 & 2016 & August \\
\hline 75 & 101 & Rock & Celon & Centrostephanus longispinus & 9 & 2016 & August \\
\hline 75 & 101 & Rock & Celon & Centrostephanus longispinus & 9 & 2016 & August \\
\hline 101 & 115 & Sand & Celon & Centrostephanus longispinus & 9 & 2016 & August \\
\hline 101 & 115 & Sand & Celon & Centrostephanus longispinus & 9 & 2016 & August \\
\hline 104 & 105 & Sand & Celon & Centrostephanus longispinus & 9 & 2016 & August \\
\hline 104 & 105 & Sand & Celon & Centrostephanus longispinus & 9 & 2016 & August \\
\hline 92 & 96 & Mud & Celon & Centrostephanus longispinus & 9 & 2016 & August \\
\hline 92 & 96 & Mud & Celon & Centrostephanus longispinus & 9 & 2016 & August \\
\hline 85 & 100 & Rock & Celon & Centrostephanus longispinus & 9.5 & 2016 & April \\
\hline 85 & 100 & Rock & Celon & Centrostephanus longispinus & 9.5 & 2016 & April \\
\hline 85 & 100 & Rock & Celon & Centrostephanus longispinus & 9.5 & 2016 & April \\
\hline 85 & 100 & Rock & Celon & Centrostephanus longispinus & 10 & 2016 & April \\
\hline 185 & 220 & Sand & Celon & Centrostephanus longispinus & 10 & 2016 & April \\
\hline 185 & 220 & Sand & Celon & Centrostephanus longispinus & 10 & 2016 & April \\
\hline 152 & 177 & Sand & Celon & Centrostephanus longispinus & 10 & 2016 & April \\
\hline 152 & 177 & Sand & Celon & Centrostephanus longispinus & 10 & 2016 & April \\
\hline 152 & 177 & Sand & Celon & Centrostephanus longispinus & 10.5 & 2016 & April \\
\hline 152 & 177 & Sand & Celon & Centrostephanus longispinus & 10.5 & 2016 & April \\
\hline 78 & 100 & Mud-Sand & Celon & Centrostephanus longispinus & 10.5 & 2016 & June \\
\hline 78 & 100 & Mud-Sand & Celon & Centrostephanus longispinus & 10.5 & 2016 & June \\
\hline 51 & 58 & Mud & Celon & Centrostephanus longispinus & 10.5 & 2016 & June \\
\hline 51 & 58 & Mud & Celon & Centrostephanus longispinus & 10.5 & 2016 & June \\
\hline 130 & 140 & Sand & Celon & Centrostephanus longispinus & 10.5 & 2016 & June \\
\hline 130 & 140 & Sand & Celon & Centrostephanus longispinus & 12 & 2016 & June \\
\hline 79 & 110 & Sand & Celon & Centrostephanus longispinus & 12 & 2016 & June \\
\hline 79 & 110 & Sand & Celon & Centrostephanus longispinus & 12 & 2016 & June \\
\hline 81 & 100 & Sand & Celon & Centrostephanus longispinus & 12 & 2016 & June \\
\hline 81 & 100 & Sand & Celon & Centrostephanus longispinus & 12 & 2016 & June \\
\hline 85 & 100 & Sand & Celon & Centrostephanus longispinus & 12 & 2016 & September \\
\hline 85 & 100 & Sand & Celon & Centrostephanus longispinus & 12 & 2016 & September \\
\hline 85 & 100 & Sand & Celon & Centrostephanus longispinus & 12 & 2016 & September \\
\hline 98 & 105 & Sand & Cicid & Cidaris cidaris & 3.5 & 2015 & October \\
\hline
\end{tabular}


S10 - H. Chammem et al.

\begin{tabular}{|c|c|c|c|c|c|c|c|}
\hline $\begin{array}{l}\text { Depth - Min } \\
(\mathrm{m})\end{array}$ & $\begin{array}{c}\text { Depth-Max } \\
(\mathrm{m})\end{array}$ & Habitats & Abr & Species & $\begin{array}{l}\text { Diameter } \\
\quad(\mathrm{cm})\end{array}$ & Year & Month \\
\hline 98 & 105 & Sand & Cicid & Cidaris cidaris & 3.5 & 2015 & October \\
\hline 98 & 105 & Sand & Cicid & Cidaris cidaris & 3.5 & 2015 & October \\
\hline 91 & 95 & Sand & Cicid & Cidaris cidaris & 3.5 & 2015 & October \\
\hline 91 & 95 & Sand & Cicid & Cidaris cidaris & 3.5 & 2015 & October \\
\hline 91 & 95 & Sand & Cicid & Cidaris cidaris & 3.5 & 2015 & October \\
\hline 91 & 95 & Sand & Cicid & Cidaris cidaris & 3.5 & 2015 & October \\
\hline 91 & 95 & Sand & Cicid & Cidaris cidaris & 3.5 & 2015 & October \\
\hline 166 & 199 & Sand & Cicid & Cidaris cidaris & 4 & 2015 & December \\
\hline 166 & 199 & Sand & Cicid & Cidaris cidaris & 4 & 2015 & December \\
\hline 166 & 199 & Sand & Cicid & Cidaris cidaris & 4 & 2015 & December \\
\hline 166 & 199 & Sand & Cicid & Cidaris cidaris & 4 & 2015 & December \\
\hline 180 & 210 & Sand & Cicid & Cidaris cidaris & 4 & 2015 & December \\
\hline 180 & 210 & Sand & Cicid & Cidaris cidaris & 4 & 2015 & December \\
\hline 180 & 210 & Sand & Cicid & Cidaris cidaris & 4 & 2015 & December \\
\hline 120 & 145 & Mud & Cicid & Cidaris cidaris & 4.5 & 2015 & December \\
\hline 120 & 145 & Mud & Cicid & Cidaris cidaris & 4.5 & 2015 & December \\
\hline 120 & 145 & Mud & Cicid & Cidaris cidaris & 4.5 & 2015 & December \\
\hline 67 & 70 & Sand & Cicid & Cidaris cidaris & 4.5 & 2015 & December \\
\hline 67 & 70 & Sand & Cicid & Cidaris cidaris & 4.5 & 2015 & December \\
\hline 80 & 111 & Mud & Cicid & Cidaris cidaris & 4.5 & 2015 & December \\
\hline 80 & 111 & Mud & Cicid & Cidaris cidaris & 4.5 & 2015 & December \\
\hline 90 & 105 & Mud-Sand & Cicid & Cidaris cidaris & 5 & 2015 & December \\
\hline 90 & 105 & Mud-Sand & Cicid & Cidaris cidaris & 5 & 2015 & December \\
\hline 90 & 105 & Mud-Sand & Cicid & Cidaris cidaris & 5 & 2015 & December \\
\hline 90 & 110 & Mud-Sand & Cicid & Cidaris cidaris & 5 & 2015 & December \\
\hline 90 & 110 & Mud-Sand & Cicid & Cidaris cidaris & 5 & 2015 & December \\
\hline 80 & 120 & Sand & Cicid & Cidaris cidaris & 5 & 2015 & December \\
\hline 80 & 120 & Sand & Cicid & Cidaris cidaris & 5 & 2015 & December \\
\hline 79 & 80 & Sand & Cicid & Cidaris cidaris & 5 & 2016 & March \\
\hline 79 & 80 & Sand & Cicid & Cidaris cidaris & 5 & 2016 & March \\
\hline 80 & 113 & Mud & Cicid & Cidaris cidaris & 5 & 2016 & March \\
\hline 80 & 113 & Mud & Cicid & Cidaris cidaris & 5 & 2016 & March \\
\hline 80 & 113 & Mud & Cicid & Cidaris cidaris & 5 & 2016 & March \\
\hline 80 & 113 & Mud & Cicid & Cidaris cidaris & 5 & 2016 & March \\
\hline 90 & 120 & Sand & Cicid & Cidaris cidaris & 5 & 2016 & March \\
\hline 90 & 120 & Sand & Cicid & Cidaris cidaris & 5 & 2016 & March \\
\hline 70 & 115 & Sand & Cicid & Cidaris cidaris & 5 & 2016 & March \\
\hline 70 & 115 & Sand & Cicid & Cidaris cidaris & 5 & 2016 & March \\
\hline 70 & 100 & Sand & Cicid & Cidaris cidaris & 5 & 2016 & March \\
\hline 70 & 100 & Sand & Cicid & Cidaris cidaris & 5 & 2016 & March \\
\hline 100 & 125 & Sand & Cicid & Cidaris cidaris & 5 & 2016 & March \\
\hline 100 & 125 & Sand & Cicid & Cidaris cidaris & 5 & 2016 & March \\
\hline 50 & 65 & Sand & Cicid & Cidaris cidaris & 6 & 2016 & March \\
\hline 50 & 65 & Sand & Cicid & Cidaris cidaris & 6 & 2016 & March \\
\hline 50 & 60 & Rock & Cicid & Cidaris cidaris & 6 & 2016 & March \\
\hline 50 & 60 & Rock & Cicid & Cidaris cidaris & 6 & 2016 & March \\
\hline 50 & 60 & Rock & Cicid & Cidaris cidaris & 6 & 2016 & August \\
\hline 50 & 60 & Rock & Cicid & Cidaris cidaris & 6 & 2016 & August \\
\hline 50 & 60 & Rock & Cicid & Cidaris cidaris & 6 & 2016 & August \\
\hline 50 & 103 & Sand & Cicid & Cidaris cidaris & 6 & 2016 & August \\
\hline 50 & 103 & Sand & Cicid & Cidaris cidaris & 6 & 2016 & August \\
\hline 77 & 96 & Mud & Cicid & Cidaris cidaris & 6 & 2016 & August \\
\hline 77 & 96 & Mud & Cicid & Cidaris cidaris & 6 & 2016 & August \\
\hline 101 & 115 & Sand & Cicid & Cidaris cidaris & 6 & 2016 & August \\
\hline 101 & 115 & Sand & Cicid & Cidaris cidaris & 6 & 2016 & August \\
\hline 104 & 105 & Sand & Cicid & Cidaris cidaris & 6 & 2016 & August \\
\hline 104 & 105 & Sand & Cicid & Cidaris cidaris & 6 & 2016 & August \\
\hline 92 & 96 & Mud & Cicid & Cidaris cidaris & 6 & 2016 & August \\
\hline 92 & 96 & Mud & Cicid & Cidaris cidaris & 6 & 2016 & August \\
\hline 85 & 100 & Rock & Cicid & Cidaris cidaris & 6 & 2016 & April \\
\hline 85 & 100 & Rock & Cicid & Cidaris cidaris & 6 & 2016 & April \\
\hline 85 & 100 & Rock & Cicid & Cidaris cidaris & 6 & 2016 & April \\
\hline 85 & 100 & Rock & Cicid & Cidaris cidaris & 6 & 2016 & April \\
\hline 152 & 177 & Sand & Cicid & Cidaris cidaris & 6 & 2016 & April \\
\hline 152 & 177 & Sand & Cicid & Cidaris cidaris & 6 & 2016 & April \\
\hline 152 & 177 & Sand & Cicid & Cidaris cidaris & 6 & 2016 & April \\
\hline 152 & 177 & Sand & Cicid & Cidaris cidaris & 6 & 2016 & April \\
\hline 152 & 177 & Sand & Cicid & Cidaris cidaris & 6 & 2016 & April \\
\hline 152 & 177 & Sand & Cicid & Cidaris cidaris & 6 & 2016 & April \\
\hline 78 & 100 & Mud-Sand & Cicid & Cidaris cidaris & 6 & 2016 & June \\
\hline 78 & 100 & Mud-Sand & Cicid & Cidaris cidaris & 6 & 2016 & June \\
\hline 51 & 58 & Mud & Cicid & Cidaris cidaris & 6 & 2016 & June \\
\hline 51 & 58 & Mud & Cicid & Cidaris cidaris & 6 & 2016 & June \\
\hline 130 & 140 & Sand & Cicid & Cidaris cidaris & 6 & 2016 & June \\
\hline 130 & 140 & Sand & Cicid & Cidaris cidaris & 6 & 2016 & June \\
\hline 70 & 95 & Rock & Cicid & Cidaris cidaris & 6 & 2016 & June \\
\hline 70 & 95 & Rock & Cicid & Cidaris cidaris & 6 & 2016 & June \\
\hline
\end{tabular}




\begin{tabular}{|c|c|c|c|c|c|c|c|}
\hline $\begin{array}{l}\text { Depth - Min } \\
(\mathrm{m})\end{array}$ & $\begin{array}{c}\text { Depth-Max } \\
(\mathrm{m})\end{array}$ & Habitats & Abr & Species & $\begin{array}{l}\text { Diameter } \\
(\mathrm{cm})\end{array}$ & Year & Month \\
\hline 79 & 110 & Sand & Cicid & Cidaris cidaris & 6 & 2016 & June \\
\hline 79 & 110 & Sand & Cicid & Cidaris cidaris & 7 & 2016 & June \\
\hline 81 & 100 & Sand & Cicid & Cidaris cidaris & 7 & 2016 & June \\
\hline 81 & 100 & Sand & Cicid & Cidaris cidaris & 7 & 2016 & June \\
\hline 85 & 100 & Sand & Cicid & Cidaris cidaris & 7 & 2016 & September \\
\hline 85 & 100 & Sand & Cicid & Cidaris cidaris & 7 & 2016 & September \\
\hline 90 & 160 & Mud-Sand & Cicid & Cidaris cidaris & 7 & 2016 & September \\
\hline 90 & 160 & Mud-Sand & Cicid & Cidaris cidaris & 7 & 2016 & September \\
\hline 72 & 90 & Rock & Cicid & Cidaris cidaris & 7 & 2016 & September \\
\hline 72 & 90 & Rock & Cicid & Cidaris cidaris & 7 & 2016 & September \\
\hline 72 & 90 & Rock & Cicid & Cidaris cidaris & 7 & 2016 & September \\
\hline 72 & 90 & Rock & Cicid & Cidaris cidaris & 7 & 2016 & September \\
\hline 78 & 84 & Rock & Cicid & Cidaris cidaris & 7 & 2016 & September \\
\hline 78 & 84 & Rock & Cicid & Cidaris cidaris & 7 & 2016 & September \\
\hline 78 & 84 & Rock & Cicid & Cidaris cidaris & 7 & 2016 & September \\
\hline 78 & 84 & Rock & Cicid & Cidaris cidaris & 7 & 2016 & September \\
\hline 135 & 170 & Sand & Cicid & Cidaris cidaris & 7 & 2016 & September \\
\hline 135 & 170 & Sand & Cicid & Cidaris cidaris & 7 & 2016 & September \\
\hline 135 & 170 & Sand & Cicid & Cidaris cidaris & 7 & 2016 & September \\
\hline 158 & 164 & Sand & Cicid & Cidaris cidaris & 7 & 2016 & July \\
\hline 158 & 164 & Sand & Cicid & Cidaris cidaris & 7 & 2016 & July \\
\hline 158 & 164 & Sand & Cicid & Cidaris cidaris & 7 & 2016 & July \\
\hline 158 & 164 & Sand & Cicid & Cidaris cidaris & 7 & 2016 & July \\
\hline 70 & 85 & Rock & Cicid & Cidaris cidaris & 7 & 2016 & July \\
\hline 70 & 85 & Rock & Cicid & Cidaris cidaris & 7 & 2016 & July \\
\hline 70 & 85 & Rock & Cicid & Cidaris cidaris & 7 & 2016 & July \\
\hline 70 & 85 & Rock & Cicid & Cidaris cidaris & 7 & 2016 & July \\
\hline 70 & 85 & Rock & Cicid & Cidaris cidaris & 7 & 2016 & July \\
\hline 70 & 85 & Rock & Cicid & Cidaris cidaris & 7 & 2016 & July \\
\hline 70 & 85 & Rock & Cicid & Cidaris cidaris & 7 & 2016 & July \\
\hline 70 & 85 & Rock & Cicid & Cidaris cidaris & 7 & 2016 & July \\
\hline 70 & 85 & Rock & Cicid & Cidaris cidaris & 7 & 2016 & July \\
\hline 70 & 85 & Rock & Cicid & Cidaris cidaris & 7.5 & 2016 & July \\
\hline 70 & 85 & Rock & Cicid & Cidaris cidaris & 7.5 & 2016 & July \\
\hline 70 & 85 & Rock & Cicid & Cidaris cidaris & 7.5 & 2016 & July \\
\hline 70 & 85 & Sand & Cicid & Cidaris cidaris & 7.5 & 2016 & July \\
\hline 68 & 94 & Mud-Sand & Gracu & Gracilechinus acutus & 12 & 2015 & October \\
\hline 70 & 100 & Sand & Gracu & Gracilechinus acutus & 7 & 2016 & March \\
\hline 70 & 100 & Sand & Gracu & Gracilechinus acutus & 7 & 2016 & March \\
\hline 70 & 100 & Sand & Gracu & Gracilechinus acutus & 7 & 2016 & March \\
\hline 100 & 125 & Sand & Gracu & Gracilechinus acutus & 7 & 2016 & March \\
\hline 100 & 125 & Sand & Gracu & Gracilechinus acutus & 8 & 2016 & March \\
\hline 100 & 125 & Sand & Gracu & Gracilechinus acutus & 8 & 2016 & March \\
\hline 50 & 60 & Rock & Gracu & Gracilechinus acutus & 8 & 2016 & August \\
\hline 50 & 60 & Rock & Gracu & Gracilechinus acutus & 9.5 & 2016 & August \\
\hline 50 & 60 & Rock & Gracu & Gracilechinus acutus & 9.5 & 2016 & August \\
\hline 50 & 60 & Rock & Gracu & Gracilechinus acutus & 11 & 2016 & August \\
\hline 50 & 103 & Sand & Gracu & Gracilechinus acutus & 11 & 2016 & August \\
\hline 50 & 103 & Sand & Gracu & Gracilechinus acutus & 11.5 & 2016 & August \\
\hline 50 & 103 & Sand & Gracu & Gracilechinus acutus & 12 & 2016 & August \\
\hline 50 & 103 & Sand & Gracu & Gracilechinus acutus & 12 & 2016 & August \\
\hline 75 & 101 & Rock & Gracu & Gracilechinus acutus & 13 & 2016 & August \\
\hline 75 & 101 & Rock & Gracu & Gracilechinus acutus & 13.5 & 2016 & August \\
\hline 75 & 101 & Rock & Gracu & Gracilechinus acutus & 14 & 2016 & August \\
\hline 75 & 101 & Rock & Gracu & Gracilechinus acutus & 14 & 2016 & August \\
\hline 0.2 & 0.6 & Algea & Paliv & Paracentrotus lividus & 1 & 2013 & \\
\hline 0.2 & 0.6 & Algea & Paliv & Paracentrotus lividus & 3 & 2013 & \\
\hline 0.2 & 0.6 & Algea & Paliv & Paracentrotus lividus & 3 & 2013 & \\
\hline 0.2 & 0.6 & Algea & Paliv & Paracentrotus lividus & 3 & 2013 & \\
\hline 0.2 & 0.6 & Algea & Paliv & Paracentrotus lividus & 3 & 2013 & \\
\hline 0.2 & 0.6 & Algea & Paliv & Paracentrotus lividus & 3 & 2014 & \\
\hline 0.2 & 0.6 & Algea & Paliv & Paracentrotus lividus & 3.5 & 2014 & \\
\hline 0.2 & 0.6 & Algea & Paliv & Paracentrotus lividus & 3.5 & 2014 & \\
\hline 0.2 & 0.6 & Algea & Paliv & Paracentrotus lividus & 3.5 & 2014 & \\
\hline 0.2 & 0.6 & Algea & Paliv & Paracentrotus lividus & 4 & 2014 & \\
\hline 0.2 & 0.6 & Algea & Paliv & Paracentrotus lividus & 4 & 2014 & \\
\hline 0.2 & 0.6 & Algea & Paliv & Paracentrotus lividus & 4 & 2014 & \\
\hline 0.2 & 0.6 & Rock & Paliv & Paracentrotus lividus & 4 & 2013 & \\
\hline 0.2 & 0.6 & Rock & Paliv & Paracentrotus lividus & 4 & 2013 & \\
\hline 0.2 & 0.6 & Rock & Paliv & Paracentrotus lividus & 4 & 2013 & \\
\hline 0.2 & 0.6 & Rock & Paliv & Paracentrotus lividus & 5 & 2013 & \\
\hline 0.2 & 0.6 & Rock & Paliv & Paracentrotus lividus & 5 & 2013 & \\
\hline 0.2 & 0.6 & Rock & Paliv & Paracentrotus lividus & 5 & 2013 & \\
\hline 3 & 6 & Sand & Paliv & Paracentrotus lividus & 5 & 2016 & May \\
\hline 3 & 6 & Sand & Paliv & Paracentrotus lividus & 5 & 2016 & May \\
\hline 3 & 6 & Sand & Paliv & Paracentrotus lividus & 5 & 2016 & May \\
\hline 3 & 6 & Sand & Paliv & Paracentrotus lividus & 5 & 2016 & May \\
\hline
\end{tabular}




\begin{tabular}{|c|c|c|c|c|c|c|c|}
\hline $\begin{array}{c}\text { Depth - Min } \\
(\mathrm{m})\end{array}$ & $\begin{array}{c}\text { Depth-Max } \\
(\mathrm{m})\end{array}$ & Habitats & Abr & Species & $\begin{array}{l}\text { Diameter } \\
(\mathrm{cm})\end{array}$ & Year & Month \\
\hline 3 & 6 & Sand & Paliv & Paracentrotus lividus & 5 & 2016 & May \\
\hline 3 & 6 & Sand & Paliv & Paracentrotus lividus & 6 & 2016 & May \\
\hline 3 & 6 & Sand & Paliv & Paracentrotus lividus & 6 & 2016 & May \\
\hline 3 & 6 & Sand & Paliv & Paracentrotus lividus & 6 & 2016 & May \\
\hline 3 & 6 & Sand & Paliv & Paracentrotus lividus & 7 & 2016 & May \\
\hline 3 & 6 & Sand & Paliv & Paracentrotus lividus & 7 & 2016 & May \\
\hline 3 & 6 & Sand & Paliv & Paracentrotus lividus & 7 & 2016 & May \\
\hline 3 & 6 & Sand & Paliv & Paracentrotus lividus & 7 & 2016 & May \\
\hline 3 & 6 & Sand & Paliv & Paracentrotus lividus & 7 & 2016 & May \\
\hline 3 & 6 & Sand & Paliv & Paracentrotus lividus & 7 & 2016 & May \\
\hline 3 & 6 & Sand & Paliv & Paracentrotus lividus & 8 & 2016 & May \\
\hline 3 & 6 & Sand & Paliv & Paracentrotus lividus & 8 & 2016 & May \\
\hline 0.2 & 0.6 & Rock & Paliv & Paracentrotus lividus & 8 & 2016 & July \\
\hline 0.2 & 0.6 & Rock & Paliv & Paracentrotus lividus & 8 & 2016 & July \\
\hline 0.2 & 0.6 & Rock & Paliv & Paracentrotus lividus & 8 & 2016 & July \\
\hline 0.2 & 0.6 & Rock & Paliv & Paracentrotus lividus & 8 & 2016 & July \\
\hline 0.2 & 0.6 & Rock & Paliv & Paracentrotus lividus & 8 & 2016 & July \\
\hline 0.2 & 0.6 & Rock & Paliv & Paracentrotus lividus & 8.5 & 2016 & July \\
\hline 0.2 & 0.6 & Rock & Paliv & Paracentrotus lividus & 8.5 & 2016 & July \\
\hline 0.2 & 0.6 & Rock & Paliv & Paracentrotus lividus & 8.5 & 2016 & July \\
\hline 0.2 & 0.6 & Rock & Paliv & Paracentrotus lividus & 9 & 2016 & July \\
\hline 0.2 & 0.6 & Rock & Paliv & Paracentrotus lividus & 9 & 2016 & July \\
\hline 0.2 & 0.6 & Sand & Paliv & Paracentrotus lividus & 9 & 2016 & July \\
\hline 0.2 & 0.6 & Sand & Paliv & Paracentrotus lividus & 9 & 2016 & July \\
\hline 0.2 & 0.6 & Sand & Paliv & Paracentrotus lividus & 9 & 2016 & July \\
\hline 0.2 & 0.6 & Sand & Paliv & Paracentrotus lividus & 9 & 2016 & July \\
\hline 0.2 & 0.6 & Algea & Spgra & Sphaerechinus granularis & 8 & 2016 & July \\
\hline 3 & 5 & Algea & Spgra & Sphaerechinus granularis & 12 & 2016 & July \\
\hline 98 & 105 & Sand & Staff & Stylocidaris affinis & 3.5 & 2015 & October \\
\hline 98 & 105 & Sand & Staff & Stylocidaris affinis & 3.5 & 2015 & October \\
\hline 98 & 105 & Sand & Staff & Stylocidaris affinis & 3.5 & 2015 & October \\
\hline 91 & 95 & Sand & Staff & Stylocidaris affinis & 3.5 & 2015 & October \\
\hline 91 & 95 & Sand & Staff & Stylocidaris affinis & 3.5 & 2015 & October \\
\hline 91 & 95 & Sand & Staff & Stylocidaris affinis & 3.5 & 2015 & October \\
\hline 91 & 95 & Sand & Staff & Stylocidaris affinis & 3.5 & 2015 & October \\
\hline 91 & 95 & Sand & Staff & Stylocidaris affinis & 3.5 & 2015 & October \\
\hline 166 & 199 & Sand & Staff & Stylocidaris affinis & 4 & 2015 & December \\
\hline 166 & 199 & Sand & Staff & Stylocidaris affinis & 4 & 2015 & December \\
\hline 166 & 199 & Sand & Staff & Stylocidaris affinis & 4 & 2015 & December \\
\hline 166 & 199 & Sand & Staff & Stylocidaris affinis & 4 & 2015 & December \\
\hline 180 & 210 & Sand & Staff & Stylocidaris affinis & 4 & 2015 & December \\
\hline 180 & 210 & Sand & Staff & Stylocidaris affinis & 4 & 2015 & December \\
\hline 180 & 210 & Sand & Staff & Stylocidaris affinis & 4 & 2015 & December \\
\hline 120 & 145 & Mud & Staff & Stylocidaris affinis & 4.5 & 2015 & December \\
\hline 120 & 145 & Mud & Staff & Stylocidaris affinis & 4.5 & 2015 & December \\
\hline 120 & 145 & Mud & Staff & Stylocidaris affinis & 4.5 & 2015 & December \\
\hline 67 & 70 & Sand & Staff & Stylocidaris affinis & 4.5 & 2015 & December \\
\hline 67 & 70 & Sand & Staff & Stylocidaris affinis & 4.5 & 2015 & December \\
\hline 80 & 111 & Mud & Staff & Stylocidaris affinis & 4.5 & 2015 & December \\
\hline 80 & 111 & Mud & Staff & Stylocidaris affinis & 4.5 & 2015 & December \\
\hline 90 & 105 & Mud-Sand & Staff & Stylocidaris affinis & 5 & 2015 & December \\
\hline 90 & 105 & Mud-Sand & Staff & Stylocidaris affinis & 5 & 2015 & December \\
\hline 90 & 105 & Mud-Sand & Staff & Stylocidaris affinis & 5 & 2015 & December \\
\hline 90 & 110 & Mud-Sand & Staff & Stylocidaris affinis & 5 & 2015 & December \\
\hline 90 & 110 & Mud-Sand & Staff & Stylocidaris affinis & 5 & 2015 & December \\
\hline 80 & 120 & Sand & Staff & Stylocidaris affinis & 5 & 2015 & December \\
\hline 80 & 120 & Sand & Staff & Stylocidaris affinis & 5 & 2015 & December \\
\hline 79 & 80 & Sand & Staff & Stylocidaris affinis & 5 & 2016 & March \\
\hline 79 & 80 & Sand & Staff & Stylocidaris affinis & 5 & 2016 & March \\
\hline 80 & 113 & Mud & Staff & Stylocidaris affinis & 5 & 2016 & March \\
\hline 80 & 113 & Mud & Staff & Stylocidaris affinis & 5 & 2016 & March \\
\hline 80 & 113 & Mud & Staff & Stylocidaris affinis & 5 & 2016 & March \\
\hline 80 & 113 & Mud & Staff & Stylocidaris affinis & 5 & 2016 & March \\
\hline 90 & 120 & Sand & Staff & Stylocidaris affinis & 5 & 2016 & March \\
\hline 90 & 120 & Sand & Staff & Stylocidaris affinis & 5 & 2016 & March \\
\hline 100 & 115 & Sand & Staff & Stylocidaris affinis & 5 & 2016 & March \\
\hline 100 & 115 & Sand & Staff & Stylocidaris affinis & 5 & 2016 & March \\
\hline 70 & 100 & Sand & Staff & Stylocidaris affinis & 5 & 2016 & March \\
\hline 70 & 100 & Sand & Staff & Stylocidaris affinis & 5 & 2016 & March \\
\hline 100 & 125 & Sand & Staff & Stylocidaris affinis & 5 & 2016 & March \\
\hline 100 & 125 & Sand & Staff & Stylocidaris affinis & 5 & 2016 & March \\
\hline 50 & 65 & Sand & Staff & Stylocidaris affinis & 6 & 2016 & March \\
\hline 50 & 65 & Sand & Staff & Stylocidaris affinis & 6 & 2016 & March \\
\hline 50 & 60 & Rock & Staff & Stylocidaris affinis & 6 & 2016 & March \\
\hline 50 & 60 & Rock & Staff & Stylocidaris affinis & 6 & 2016 & March \\
\hline 50 & 60 & Rock & Staff & Stylocidaris affinis & 6 & 2016 & August \\
\hline 50 & 60 & Rock & Staff & Stylocidaris affinis & 6 & 2016 & August \\
\hline
\end{tabular}




\begin{tabular}{|c|c|c|c|c|c|c|c|}
\hline $\begin{array}{l}\text { Depth - Min } \\
(\mathrm{m})\end{array}$ & $\begin{array}{c}\text { Depth-Max } \\
(\mathrm{m})\end{array}$ & Habitats & Abr & Species & $\begin{array}{l}\text { Diameter } \\
(\mathrm{cm})\end{array}$ & Year & Month \\
\hline 50 & 60 & Rock & Staff & Stylocidaris affinis & 6 & 2016 & August \\
\hline 50 & 103 & Sand & Staff & Stylocidaris affinis & 6 & 2016 & August \\
\hline 50 & 103 & Sand & Staff & Stylocidaris affinis & 6 & 2016 & August \\
\hline 77 & 96 & Mud & Staff & Stylocidaris affinis & 6 & 2016 & August \\
\hline 77 & 96 & Mud & Staff & Stylocidaris affinis & 6 & 2016 & August \\
\hline 101 & 115 & Sand & Staff & Stylocidaris affinis & 6 & 2016 & August \\
\hline 101 & 115 & Sand & Staff & Stylocidaris affinis & 6 & 2016 & August \\
\hline 104 & 105 & Sand & Staff & Stylocidaris affinis & 6 & 2016 & August \\
\hline 104 & 105 & Sand & Staff & Stylocidaris affinis & 6 & 2016 & August \\
\hline 92 & 96 & Mud & Staff & Stylocidaris affinis & 6 & 2016 & August \\
\hline 92 & 96 & Mud & Staff & Stylocidaris affinis & 6 & 2016 & August \\
\hline 85 & 100 & Rock & Staff & Stylocidaris affinis & 6 & 2016 & April \\
\hline 85 & 100 & Rock & Staff & Stylocidaris affinis & 6 & 2016 & April \\
\hline 85 & 100 & Rock & Staff & Stylocidaris affinis & 6 & 2016 & April \\
\hline 85 & 100 & Rock & Staff & Stylocidaris affinis & 6 & 2016 & April \\
\hline 152 & 177 & Sand & Staff & Stylocidaris affinis & 6 & 2016 & April \\
\hline 152 & 177 & Sand & Staff & Stylocidaris affinis & 6 & 2016 & April \\
\hline 152 & 177 & Sand & Staff & Stylocidaris affinis & 6 & 2016 & April \\
\hline 152 & 177 & Sand & Staff & Stylocidaris affinis & 6 & 2016 & April \\
\hline 152 & 177 & Sand & Staff & Stylocidaris affinis & 6 & 2016 & April \\
\hline 152 & 177 & Sand & Staff & Stylocidaris affinis & 6 & 2016 & April \\
\hline 78 & 100 & Mud-Sand & Staff & Stylocidaris affinis & 6 & 2016 & June \\
\hline 78 & 100 & Mud-Sand & Staff & Stylocidaris affinis & 6 & 2016 & June \\
\hline 51 & 58 & Mud & Staff & Stylocidaris affinis & 6 & 2016 & June \\
\hline 51 & 58 & Mud & Staff & Stylocidaris affinis & 6 & 2016 & June \\
\hline 130 & 140 & Sand & Staff & Stylocidaris affinis & 6 & 2016 & June \\
\hline 130 & 140 & Sand & Staff & Stylocidaris affinis & 6 & 2016 & June \\
\hline 70 & 95 & Rock & Staff & Stylocidaris affinis & 6 & 2016 & June \\
\hline 70 & 95 & Rock & Staff & Stylocidaris affinis & 6 & 2016 & June \\
\hline 79 & 110 & Sand & Staff & Stylocidaris affinis & 6 & 2016 & June \\
\hline 79 & 110 & Sand & Staff & Stylocidaris affinis & 7 & 2016 & June \\
\hline 81 & 100 & Sand & Staff & Stylocidaris affinis & 7 & 2016 & June \\
\hline 81 & 100 & Sand & Staff & Stylocidaris affinis & 7 & 2016 & June \\
\hline 85 & 100 & Sand & Staff & Stylocidaris affinis & 7 & 2016 & September \\
\hline 85 & 100 & Sand & Staff & Stylocidaris affinis & 7 & 2016 & September \\
\hline 90 & 160 & Mud-Sand & Staff & Stylocidaris affinis & 7 & 2016 & September \\
\hline 90 & 160 & Mud-Sand & Staff & Stylocidaris affinis & 7 & 2016 & September \\
\hline 72 & 90 & Rock & Staff & Stylocidaris affinis & 7 & 2016 & September \\
\hline 72 & 90 & Rock & Staff & Stylocidaris affinis & 7 & 2016 & September \\
\hline 72 & 90 & Rock & Staff & Stylocidaris affinis & 7 & 2016 & September \\
\hline 72 & 90 & Rock & Staff & Stylocidaris affinis & 7 & 2016 & September \\
\hline 78 & 84 & Rock & Staff & Stylocidaris affinis & 7 & 2016 & September \\
\hline 78 & 84 & Rock & Staff & Stylocidaris affinis & 7 & 2016 & September \\
\hline 78 & 84 & Rock & Staff & Stylocidaris affinis & 7 & 2016 & September \\
\hline 78 & 84 & Rock & Staff & Stylocidaris affinis & 7 & 2016 & September \\
\hline 135 & 170 & Sand & Staff & Stylocidaris affinis & 7 & 2016 & September \\
\hline 135 & 170 & Sand & Staff & Stylocidaris affinis & 7 & 2016 & September \\
\hline 135 & 170 & Sand & Staff & Stylocidaris affinis & 7 & 2016 & September \\
\hline 158 & 164 & Sand & Staff & Stylocidaris affinis & 7 & 2016 & July \\
\hline 158 & 164 & Sand & Staff & Stylocidaris affinis & 7 & 2016 & July \\
\hline 158 & 164 & Sand & Staff & Stylocidaris affinis & 7 & 2016 & July \\
\hline 158 & 164 & Sand & Staff & Stylocidaris affinis & 7 & 2016 & July \\
\hline 70 & 85 & Rock & Staff & Stylocidaris affinis & 7 & 2016 & July \\
\hline 70 & 85 & Rock & Staff & Stylocidaris affinis & 7 & 2016 & July \\
\hline 70 & 85 & Rock & Staff & Stylocidaris affinis & 7 & 2016 & July \\
\hline 70 & 85 & Rock & Staff & Stylocidaris affinis & 7 & 2016 & July \\
\hline 70 & 85 & Rock & Staff & Stylocidaris affinis & 7 & 2016 & July \\
\hline 70 & 85 & Rock & Staff & Stylocidaris affinis & 7 & 2016 & July \\
\hline 70 & 85 & Rock & Staff & Stylocidaris affinis & 7 & 2016 & July \\
\hline 70 & 85 & Rock & Staff & Stylocidaris affinis & 7 & 2016 & July \\
\hline 70 & 85 & Rock & Staff & Stylocidaris affinis & 7 & 2016 & July \\
\hline 70 & 85 & Rock & Staff & Stylocidaris affinis & 7.5 & 2016 & July \\
\hline 70 & 85 & Rock & Staff & Stylocidaris affinis & 7.5 & 2016 & July \\
\hline 70 & 85 & Rock & Staff & Stylocidaris affinis & 7.5 & 2016 & July \\
\hline 70 & 85 & Sand & Staff & Stylocidaris affinis & 7.5 & 2016 & July \\
\hline 70 & 85 & Sand & Staff & Stylocidaris affinis & 8 & 2016 & August \\
\hline 70 & 85 & Sand & Stpur & Spatangus purpureus & 8 & 2015 & \\
\hline 3 & 5 & Sand & Hesyr & Hemiocnus syracusanus & 5 & 2016 & \\
\hline 0.2 & 0.3 & Rock & Hoimp & Holothuria (Thymiosycia) impatiens & 13 & 2016 & \\
\hline 3 & 8 & Sand & Hopol & Holothuria (Roweothuria) poli & 10 & 2016 & \\
\hline 3 & 8 & Sand & Hopol & Holothuria (Roweothuria) poli & 10 & 2016 & \\
\hline 3 & 8 & Sand & Hopol & Holothuria (Roweothuria) poli & 10 & 2016 & \\
\hline 3 & 8 & Sand & Hopol & Holothuria (Roweothuria) poli & 10 & 2016 & \\
\hline 3 & 8 & Sand & Hopol & Holothuria (Roweothuria) poli & 10 & 2016 & \\
\hline 3 & 8 & Sand & Hopol & Holothuria (Roweothuria) poli & 10 & 2016 & \\
\hline 3 & 8 & Sand & Hotub & Holothuria (Holothuria) tubulosa & 8 & 2016 & \\
\hline 3 & 8 & Sand & Hotub & Holothuria (Holothuria) tubulosa & 8 & 2016 & \\
\hline
\end{tabular}




\begin{tabular}{|c|c|c|c|c|c|c|c|}
\hline $\begin{array}{l}\text { Depth - Min } \\
(\mathrm{m})\end{array}$ & $\begin{array}{c}\text { Depth-Max } \\
(\mathrm{m})\end{array}$ & Habitats & Abr & Species & $\begin{array}{l}\text { Diameter } \\
(\mathrm{cm})\end{array}$ & Year & Month \\
\hline 3 & 8 & Sand & Hotub & Holothuria (Holothuria) tubulosa & 8 & 2016 & \\
\hline 3 & 8 & Sand & Homam & Holothuria (Holothuria) mammata & 7 & 2016 & \\
\hline 3 & 8 & Sand & Homam & Holothuria (Holothuria) mammata & 9 & 2016 & \\
\hline 0.2 & 0.6 & Algea & Hopol & Holothuria (Roweothuria) poli & 10 & 2016 & \\
\hline 0.2 & 0.6 & Algea & Hopol & Holothuria (Roweothuria) poli & 10 & 2016 & \\
\hline 0.2 & 0.6 & Algea & Hopol & Holothuria (Roweothuria) poli & 10 & 2016 & \\
\hline 0.2 & 0.6 & Algea & Hopol & Holothuria (Roweothuria) poli & 10.5 & 2016 & \\
\hline 0.2 & 0.6 & Algea & Hopol & Holothuria (Roweothuria) poli & 10.5 & 2016 & \\
\hline 0.2 & 0.6 & Algea & Hopol & Holothuria (Roweothuria) poli & 10.5 & 2016 & \\
\hline 0.2 & 0.6 & Algea & Hopol & Holothuria (Roweothuria) poli & 11 & 2016 & \\
\hline 0.2 & 0.6 & Algea & Hopol & Holothuria (Roweothuria) poli & 11 & 2016 & \\
\hline 0.2 & 0.6 & Algea & Hopol & Holothuria (Roweothuria) poli & 11 & 2016 & \\
\hline 0.2 & 0.6 & Algea & Hopol & Holothuria (Roweothuria) poli & 11 & 2016 & \\
\hline 0.2 & 0.6 & Algea & Hopol & Holothuria (Roweothuria) poli & 11 & 2016 & \\
\hline 0.2 & 0.6 & Algea & Hopol & Holothuria (Roweothuria) poli & 11 & 2016 & \\
\hline 0.2 & 0.6 & Algea & Hopol & Holothuria (Roweothuria) poli & 11 & 2016 & \\
\hline 0.2 & 0.6 & Algea & Hopol & Holothuria (Roweothuria) poli & 12 & 2016 & \\
\hline 0.2 & 0.6 & Algea & Hopol & Holothuria (Roweothuria) poli & 12 & 2016 & \\
\hline 0.2 & 0.6 & Algea & Hopol & Holothuria (Roweothuria) poli & 12 & 2016 & \\
\hline 0.2 & 0.6 & Algea & Hosan & Holothuria (Platyperona) sanctori & 10 & 2016 & \\
\hline 0.2 & 0.6 & Algea & Hosan & Holothuria (Platyperona) sanctori & 10 & 2016 & \\
\hline 0.2 & 0.6 & Algea & Hosan & Holothuria (Platyperona) sanctori & 10 & 2016 & \\
\hline 0.2 & 0.6 & Algea & Hosan & Holothuria (Platyperona) sanctori & 10 & 2016 & \\
\hline 0.2 & 0.6 & Algea & Hosan & Holothuria (Platyperona) sanctori & 10 & 2016 & \\
\hline 0.2 & 0.6 & Algea & Hosan & Holothuria (Platyperona) sanctori & 10 & 2016 & \\
\hline 0.2 & 0.6 & Algea & Hosan & Holothuria (Platyperona) sanctori & 11 & 2016 & \\
\hline 0.2 & 0.6 & Algea & Hosan & Holothuria (Platyperona) sanctori & 11 & 2016 & \\
\hline 3 & 6 & Sand & Hotub & Holothuria (Holothuria) tubulosa & 11 & 2016 & May \\
\hline 3 & 6 & Sand & Hotub & Holothuria (Holothuria) tubulosa & 11 & 2016 & May \\
\hline 3 & 6 & Sand & Hotub & Holothuria (Holothuria) tubulosa & 11 & 2016 & May \\
\hline 3 & 6 & Sand & Hotub & Holothuria (Holothuria) tubulosa & 12 & 2016 & May \\
\hline 3 & 6 & Sand & Hotub & Holothuria (Holothuria) tubulosa & 12 & 2016 & May \\
\hline 3 & 6 & Sand & Hotub & Holothuria (Holothuria) tubulosa & 12 & 2016 & May \\
\hline 0.2 & 0.6 & Sand & Hotub & Holothuria (Holothuria) tubulosa & 12 & 2016 & July \\
\hline 0.2 & 0.6 & Sand & Hotub & Holothuria (Holothuria) tubulosa & 12 & 2016 & July \\
\hline 0.2 & 0.6 & Sand & Hotub & Holothuria (Holothuria) tubulosa & 12 & 2016 & July \\
\hline 0.2 & 0.6 & Algea & Hotub & Holothuria (Holothuria) tubulosa & 8 & 2013 & \\
\hline 0.2 & 0.6 & Algea & Hotub & Holothuria (Holothuria) tubulosa & 8 & 2013 & \\
\hline 0.2 & 0.6 & Algea & Hotub & Holothuria (Holothuria) tubulosa & 8 & 2013 & \\
\hline 0.2 & 0.6 & Algea & Hotub & Holothuria (Holothuria) tubulosa & 8 & 2013 & \\
\hline 0.2 & 0.6 & Algea & Hotub & Holothuria (Holothuria) tubulosa & 8.5 & 2013 & \\
\hline 0.2 & 0.6 & Algea & Hotub & Holothuria (Holothuria) tubulosa & 8.5 & 2013 & \\
\hline 0.2 & 0.6 & Algea & Hotub & Holothuria (Holothuria) tubulosa & 8.5 & 2013 & \\
\hline 0.2 & 0.6 & Algea & Hotub & Holothuria (Holothuria) tubulosa & 8.5 & 2013 & \\
\hline 0.2 & 0.6 & Algea & Hotub & Holothuria (Holothuria) tubulosa & 8.5 & 2013 & \\
\hline 0.2 & 0.6 & Algea & Hotub & Holothuria (Holothuria) tubulosa & 8.5 & 2013 & \\
\hline 0.2 & 0.6 & Algea & Hotub & Holothuria (Holothuria) tubulosa & 8.5 & 2013 & \\
\hline 0.2 & 0.6 & Algea & Hotub & Holothuria (Holothuria) tubulosa & 8.5 & 2014 & \\
\hline 3 & 8 & Sand & Hotub & Holothuria (Holothuria) tubulosa & 10 & 2014 & \\
\hline 3 & 8 & Sand & Hotub & Holothuria (Holothuria) tubulosa & 10 & 2014 & \\
\hline 3 & 8 & Sand & Hotub & Holothuria (Holothuria) tubulosa & 10 & 2014 & \\
\hline 3 & 8 & Sand & Hotub & Holothuria (Holothuria) tubulosa & 10 & 2014 & \\
\hline 3 & 8 & Sand & Hotub & Holothuria (Holothuria) tubulosa & 10 & 2014 & \\
\hline 3 & 8 & Sand & Hotub & Holothuria (Holothuria) tubulosa & 12 & 2014 & \\
\hline 3 & 8 & Sand & Hotub & Holothuria (Holothuria) tubulosa & 12 & 2014 & \\
\hline 185 & 220 & Sand & Hotub & Holothuria (Holothuria) tubulosa & 12 & 2014 & \\
\hline 185 & 220 & Sand & Hotub & Holothuria (Holothuria) tubulosa & 12 & 2014 & \\
\hline 79 & 110 & Sand & Hotub & Holothuria (Holothuria) tubulosa & 12 & 2014 & \\
\hline 79 & 110 & Sand & Hotub & Holothuria (Holothuria) tubulosa & 12 & 2014 & \\
\hline 85 & 100 & Sand & Hotub & Holothuria (Holothuria) tubulosa & 13 & 2014 & \\
\hline 90 & 160 & Mud-Sand & Hotub & Holothuria (Holothuria) tubulosa & 13 & 2013 & \\
\hline 90 & 160 & Mud-Sand & Hotub & Holothuria (Holothuria) tubulosa & 13 & 2013 & \\
\hline 3 & 8 & Sand & Hotub & Holothuria (Holothuria) tubulosa & 13 & 2013 & \\
\hline 3 & 8 & Sand & Hotub & Holothuria (Holothuria) tubulosa & 13 & 2013 & \\
\hline 3 & 8 & Sand & Hotub & Holothuria (Holothuria) tubulosa & 14 & 2013 & \\
\hline 3 & 8 & Sand & Hotub & Holothuria (Holothuria) tubulosa & 15 & 2013 & \\
\hline 3 & 8 & Sand & Hotub & Holothuria (Holothuria) tubulosa & 5 & 2013 & \\
\hline 98 & 105 & Sand & Leter & Leptopentacta tergistina & 5 & 2015 & October \\
\hline 120 & 145 & Mud & Leter & Leptopentacta tergistina & 5 & 2015 & October \\
\hline 79 & 80 & Sand & Leter & Leptopentacta tergistina & 5 & 2016 & March \\
\hline 77 & 96 & Mud & Leter & Leptopentacta tergistina & 5 & 2015 & December \\
\hline 101 & 115 & Sand & Leter & Leptopentacta tergistina & 5 & 2016 & August \\
\hline 60 & 96 & Sand & Leelo & Leptopentacta elongata & & 2015 & \\
\hline 85 & 98 & Mud & Pareg & Parastichopus regalis & 11 & 2015 & October \\
\hline 85 & 98 & Mud & Pareg & Parastichopus regalis & 11 & 2015 & October \\
\hline 85 & 98 & Mud & Pareg & Parastichopus regalis & 11 & 2015 & October \\
\hline 98 & 105 & Sand & Pareg & Parastichopus regalis & 11 & 2015 & October \\
\hline
\end{tabular}




\begin{tabular}{|c|c|c|c|c|c|c|c|}
\hline $\begin{array}{l}\text { Depth - Min } \\
\text { (m) }\end{array}$ & $\begin{array}{c}\text { Depth-Max } \\
(\mathrm{m})\end{array}$ & Habitats & Abr & Species & $\begin{array}{l}\text { Diameter } \\
(\mathrm{cm})\end{array}$ & Year & Month \\
\hline 98 & 105 & Sand & Pareg & Parastichopus regalis & 11 & 2015 & October \\
\hline 98 & 105 & Sand & Pareg & Parastichopus regalis & 11 & 2015 & October \\
\hline 98 & 105 & Sand & Pareg & Parastichopus regalis & 11 & 2015 & October \\
\hline 91 & 95 & Sand & Pareg & Parastichopus regalis & 11 & 2015 & October \\
\hline 91 & 95 & Sand & Pareg & Parastichopus regalis & 11 & 2015 & December \\
\hline 120 & 145 & Mud & Pareg & Parastichopus regalis & 11 & 2015 & December \\
\hline 120 & 145 & Mud & Pareg & Parastichopus regalis & 11.5 & 2015 & December \\
\hline 120 & 145 & Mud & Pareg & Parastichopus regalis & 11.5 & 2015 & December \\
\hline 67 & 70 & Sand & Pareg & Parastichopus regalis & 11.5 & 2015 & December \\
\hline 67 & 70 & Sand & Pareg & Parastichopus regalis & 12 & 2015 & December \\
\hline 115 & 130 & Sand & Pareg & Parastichopus regalis & 12 & 2015 & December \\
\hline 115 & 130 & Sand & Pareg & Parastichopus regalis & 12 & 2015 & December \\
\hline 90 & 110 & Mud-Sand & Pareg & Parastichopus regalis & 12 & 2016 & March \\
\hline 90 & 110 & Mud-Sand & Pareg & Parastichopus regalis & 12 & 2016 & March \\
\hline 90 & 110 & Mud-Sand & Pareg & Parastichopus regalis & 12 & 2016 & March \\
\hline 50 & 60 & Rock & Pareg & Parastichopus regalis & 13 & 2016 & March \\
\hline 50 & 60 & Rock & Pareg & Parastichopus regalis & 13 & 2016 & March \\
\hline 50 & 60 & Rock & Pareg & Parastichopus regalis & 13 & 2016 & March \\
\hline 50 & 60 & Rock & Pareg & Parastichopus regalis & 13.5 & 2016 & March \\
\hline 50 & 60 & Rock & Pareg & Parastichopus regalis & 13.5 & 2016 & August \\
\hline 50 & 60 & Rock & Pareg & Parastichopus regalis & 13.5 & 2016 & August \\
\hline 50 & 60 & Rock & Pareg & Parastichopus regalis & 14 & 2016 & August \\
\hline 50 & 60 & Rock & Pareg & Parastichopus regalis & 14 & 2016 & August \\
\hline 77 & 96 & Mud & Pareg & Parastichopus regalis & 14 & 2016 & August \\
\hline 77 & 96 & Mud & Pareg & Parastichopus regalis & 14 & 2016 & August \\
\hline 77 & 96 & Mud & Pareg & Parastichopus regalis & 14 & 2016 & August \\
\hline 77 & 96 & Mud & Pareg & Parastichopus regalis & 14 & 2016 & August \\
\hline 160 & 194 & Mud & Pareg & Parastichopus regalis & 14 & 2016 & July \\
\hline 160 & 194 & Mud & Pareg & Parastichopus regalis & 14 & 2016 & July \\
\hline 160 & 194 & Mud & Pareg & Parastichopus regalis & 14 & 2016 & July \\
\hline 180 & 192 & Mud & Pareg & Parastichopus regalis & 15 & 2016 & July \\
\hline 180 & 192 & Mud & Pareg & Parastichopus regalis & 15 & 2016 & July \\
\hline 175 & 193 & Sand & Pareg & Parastichopus regalis & 15 & 2016 & July \\
\hline 175 & 193 & Sand & Pareg & Parastichopus regalis & 15 & 2016 & July \\
\hline 175 & 193 & Sand & Pareg & Parastichopus regalis & 15 & 2016 & July \\
\hline 176 & 179 & Mud-Sand & Pareg & Parastichopus regalis & 15 & 2016 & July \\
\hline 176 & 179 & Mud-Sand & Pareg & Parastichopus regalis & 15 & 2016 & July \\
\hline 176 & 179 & Mud-Sand & Pareg & Parastichopus regalis & 15 & 2016 & July \\
\hline 142 & 163 & Mud & Pareg & Parastichopus regalis & 16 & 2016 & July \\
\hline 142 & 163 & Mud & Pareg & Parastichopus regalis & 16 & 2016 & July \\
\hline 142 & 163 & Mud & Pareg & Parastichopus regalis & 16 & 2016 & July \\
\hline 142 & 163 & Mud & Pareg & Parastichopus regalis & 16 & 2016 & July \\
\hline 142 & 163 & Mud & Pareg & Parastichopus regalis & 16 & 2016 & July \\
\hline 181 & 186 & Mud-Sand & Pareg & Parastichopus regalis & 16 & 2016 & July \\
\hline 181 & 186 & Mud-Sand & Pareg & Parastichopus regalis & 16 & 2016 & July \\
\hline 179 & 185 & Sand & Pareg & Parastichopus regalis & 16 & 2016 & July \\
\hline 179 & 185 & Sand & Pareg & Parastichopus regalis & 16 & 2016 & July \\
\hline 179 & 185 & Sand & Pareg & Parastichopus regalis & 16 & 2016 & July \\
\hline 181 & 190 & Sand & Pareg & Parastichopus regalis & 16 & 2016 & July \\
\hline 181 & 190 & Sand & Pareg & Parastichopus regalis & 16 & 2016 & July \\
\hline 181 & 190 & Sand & Pareg & Parastichopus regalis & 16 & 2016 & July \\
\hline 181 & 190 & Sand & Pareg & Parastichopus regalis & 17 & 2016 & July \\
\hline 181 & 190 & Sand & Pareg & Parastichopus regalis & 17 & 2016 & July \\
\hline 0.2 & 0.6 & Rock & Pareg & Parastichopus regalis & 17 & 2014 & \\
\hline 0.45 & 0.55 & Rock & Amsqu & Amphipholis squamata & 0.5 & 2013 & \\
\hline 0.45 & 0.55 & Rock & Amsqu & Amphipholis squamata & 0.5 & 2013 & \\
\hline 0.45 & 0.55 & Rock & Amsqu & Amphipholis squamata & 0.5 & 2013 & \\
\hline 0.45 & 0.55 & Rock & Amsqu & Amphipholis squamata & 0.5 & 2013 & \\
\hline 0.45 & 0.55 & Rock & Amsqu & Amphipholis squamata & 0.5 & 2013 & \\
\hline 0.45 & 0.55 & Rock & Amsqu & Amphipholis squamata & 0.5 & 2013 & \\
\hline 0.45 & 0.55 & Rock & Amsqu & Amphipholis squamata & 0.5 & 2013 & \\
\hline 0.45 & 0.55 & Rock & Amsqu & Amphipholis squamata & 0.5 & 2014 & \\
\hline 0.45 & 0.55 & Rock & Amsqu & Amphipholis squamata & 0.6 & 2014 & \\
\hline 0.45 & 0.55 & Rock & Amsqu & Amphipholis squamata & 0.6 & 2014 & \\
\hline 0.45 & 0.55 & Rock & Amsqu & Amphipholis squamata & 0.6 & 2014 & \\
\hline 0.45 & 0.55 & Rock & Amsqu & Amphipholis squamata & 0.6 & 2014 & \\
\hline 0.45 & 0.55 & Rock & Amsqu & Amphipholis squamata & 0.6 & 2014 & \\
\hline 0.45 & 0.55 & Rock & Amsqu & Amphipholis squamata & 0.6 & 2014 & \\
\hline 0.45 & 0.55 & Rock & Amsqu & Amphipholis squamata & 0.6 & 2014 & \\
\hline 0.45 & 0.55 & Rock & Amsqu & Amphipholis squamata & 0.6 & 2014 & \\
\hline 98 & 105 & Sand & Asmed & Astrospartus mediterraneus & 6 & 2015 & October \\
\hline 158 & 164 & Gorgonian & Opset & Ophiacantha setosa & 0.3 & 2015 & November \\
\hline 158 & 164 & Gorgonian & Opset & Ophiacantha setosa & 0.3 & 2015 & November \\
\hline 158 & 164 & Gorgonian & Opset & Ophiacantha setosa & 0.3 & 2015 & November \\
\hline 158 & 164 & Gorgonian & Opset & Ophiacantha setosa & 0.3 & 2015 & November \\
\hline 158 & 164 & Gorgonian & Opset & Ophiacantha setosa & 0.3 & 2015 & November \\
\hline 158 & 164 & Gorgonian & Opset & Ophiacantha setosa & 0.5 & 2015 & November \\
\hline
\end{tabular}


S16 - H. Chammem et al.

\begin{tabular}{|c|c|c|c|c|c|c|c|}
\hline $\begin{array}{c}\text { Depth - Min } \\
(\mathrm{m})\end{array}$ & $\begin{array}{c}\text { Depth-Max } \\
(\mathrm{m})\end{array}$ & Habitats & Abr & Species & $\begin{array}{c}\text { Diameter } \\
(\mathrm{cm})\end{array}$ & Year & Month \\
\hline 158 & 164 & Gorgonian & Opset & Ophiacantha setosa & 0.5 & 2015 & November \\
\hline 158 & 164 & Gorgonian & Opset & Ophiacantha setosa & 0.5 & 2015 & November \\
\hline 158 & 164 & Gorgonian & Opset & Ophiacantha setosa & 0.5 & 2015 & November \\
\hline 158 & 164 & Gorgonian & Opset & Ophiacantha setosa & 0.5 & 2015 & November \\
\hline 158 & 164 & Gorgonian & Opset & Ophiacantha setosa & 0.6 & 2015 & November \\
\hline 158 & 164 & Gorgonian & Opset & Ophiacantha setosa & 0.6 & 2016 & April \\
\hline 158 & 164 & Gorgonian & Opset & Ophiacantha setosa & 0.6 & 2016 & April \\
\hline 158 & 164 & Gorgonian & Opset & Ophiacantha setosa & 0.6 & 2016 & April \\
\hline 158 & 164 & Gorgonian & Opset & Ophiacantha setosa & 0.6 & 2016 & April \\
\hline 158 & 164 & Gorgonian & Opset & Ophiacantha setosa & 0.6 & 2016 & April \\
\hline 158 & 164 & Gorgonian & Opset & Ophiacantha setosa & 0.6 & 2016 & April \\
\hline 158 & 164 & Gorgonian & Opset & Ophiacantha setosa & 0.6 & 2016 & April \\
\hline 158 & 164 & Gorgonian & Opset & Ophiacantha setosa & 0.6 & 2016 & April \\
\hline 158 & 164 & Gorgonian & Opset & Ophiacantha setosa & 0.6 & 2016 & April \\
\hline 158 & 164 & Gorgonian & Opset & Ophiacantha setosa & 0.6 & 2016 & April \\
\hline 158 & 164 & Gorgonian & Opset & Ophiacantha setosa & 0.6 & 2016 & April \\
\hline 158 & 164 & Gorgonian & Opset & Ophiacantha setosa & 0.6 & 2016 & April \\
\hline 158 & 164 & Gorgonian & Opset & Ophiacantha setosa & 0.6 & 2016 & April \\
\hline 158 & 164 & Gorgonian & Opset & Ophiacantha setosa & 0.6 & 2016 & April \\
\hline 158 & 164 & Gorgonian & Opset & Ophiacantha setosa & 0.6 & 2016 & April \\
\hline 158 & 164 & Gorgonian & Opset & Ophiacantha setosa & 0.6 & 2016 & July \\
\hline 158 & 164 & Gorgonian & Opset & Ophiacantha setosa & 0.6 & 2016 & July \\
\hline 158 & 164 & Gorgonian & Opset & Ophiacantha setosa & 0.6 & 2016 & July \\
\hline 158 & 164 & Gorgonian & Opset & Ophiacantha setosa & 0.6 & 2016 & July \\
\hline 70 & 85 & Gorgonian & Opset & Ophiacantha setosa & 0.6 & 2016 & July \\
\hline 70 & 85 & Gorgonian & Opset & Ophiacantha setosa & 0.6 & 2016 & July \\
\hline 70 & 85 & Gorgonian & Opset & Ophiacantha setosa & 0.6 & 2016 & July \\
\hline 70 & 85 & Gorgonian & Opset & Ophiacantha setosa & 0.6 & 2016 & July \\
\hline 70 & 85 & Gorgonian & Opset & Ophiacantha setosa & 0.6 & 2016 & July \\
\hline 70 & 85 & Gorgonian & Opset & Ophiacantha setosa & 0.6 & 2016 & July \\
\hline 70 & 85 & Gorgonian & Opset & Ophiacantha setosa & 0.6 & 2016 & July \\
\hline 70 & 85 & Gorgonian & Opset & Ophiacantha setosa & 0.6 & 2016 & July \\
\hline 70 & 85 & Gorgonian & Opset & Ophiacantha setosa & 0.6 & 2016 & July \\
\hline 70 & 85 & Gorgonian & Opset & Ophiacantha setosa & 0.6 & 2016 & July \\
\hline 70 & 85 & Gorgonian & Opset & Ophiacantha setosa & 0.6 & 2016 & July \\
\hline 70 & 85 & Gorgonian & Opset & Ophiacantha setosa & 0.6 & 2016 & July \\
\hline 70 & 85 & Gorgonian & Opset & Ophiacantha setosa & 0.6 & 2016 & July \\
\hline 70 & 85 & Gorgonian & Opset & Ophiacantha setosa & 0.9 & 2016 & July \\
\hline 70 & 85 & Gorgonian & Opset & Ophiacantha setosa & 0.9 & 2016 & July \\
\hline 70 & 85 & Gorgonian & Opset & Ophiacantha setosa & 0.9 & 2016 & July \\
\hline 70 & 85 & Gorgonian & Opset & Ophiacantha setosa & 0.9 & 2016 & July \\
\hline 70 & 85 & Gorgonian & Opset & Ophiacantha setosa & 0.9 & 2016 & July \\
\hline 70 & 85 & Gorgonian & Opset & Ophiacantha setosa & 0.9 & 2016 & July \\
\hline 70 & 85 & Gorgonian & Opset & Ophiacantha setosa & 0.9 & 2016 & July \\
\hline 70 & 85 & Gorgonian & Opset & Ophiacantha setosa & 0.9 & 2016 & July \\
\hline 70 & 85 & Gorgonian & Opset & Ophiacantha setosa & 0.9 & 2016 & July \\
\hline 70 & 85 & Gorgonian & Opset & Ophiacantha setosa & 0.9 & 2016 & July \\
\hline 70 & 85 & Gorgonian & Opset & Ophiacantha setosa & 0.9 & 2016 & July \\
\hline 0.45 & 0.65 & Rock & Opvir & Ophiactis virens & 0.2 & 2012 & \\
\hline 0.45 & 0.65 & Rock & Opvir & Ophiactis virens & 0.2 & 2012 & \\
\hline 0.45 & 0.65 & Rock & Opvir & Ophiactis virens & 0.2 & 2012 & \\
\hline 0.45 & 0.65 & Rock & Opvir & Ophiactis virens & 0.2 & 2012 & \\
\hline 0.45 & 0.65 & Rock & Opvir & Ophiactis virens & 0.2 & 2012 & \\
\hline 0.45 & 0.65 & Rock & Opvir & Ophiactis virens & 0.2 & 2012 & \\
\hline 0.45 & 0.65 & Rock & Opvir & Ophiactis virens & 0.2 & 2012 & \\
\hline 0.45 & 0.65 & Rock & Opvir & Ophiactis virens & 0.2 & 2012 & \\
\hline 0.45 & 0.65 & Rock & Opvir & Ophiactis virens & 0.2 & 2012 & \\
\hline 0.45 & 0.65 & Rock & Opvir & Ophiactis virens & 0.2 & 2012 & \\
\hline 0.45 & 0.65 & Rock & Opvir & Ophiactis virens & 0.2 & 2012 & \\
\hline 0.45 & 0.65 & Rock & Opvir & Ophiactis virens & 0.2 & 2012 & \\
\hline 0.45 & 0.65 & Rock & Opvir & Ophiactis virens & 0.2 & 2012 & \\
\hline 0.45 & 0.65 & Rock & Opvir & Ophiactis virens & 0.2 & 2012 & \\
\hline 0.45 & 0.65 & Rock & Opvir & Ophiactis virens & 0.2 & 2012 & \\
\hline 0.45 & 0.65 & Rock & Opvir & Ophiactis virens & 0.2 & 2012 & \\
\hline 0.45 & 0.65 & Rock & Opvir & Ophiactis virens & 0.2 & 2012 & \\
\hline 0.45 & 0.65 & Rock & Opvir & Ophiactis virens & 0.2 & 2012 & \\
\hline 0.45 & 0.65 & Rock & Opvir & Ophiactis virens & 0.2 & 2012 & \\
\hline 0.45 & 0.65 & Rock & Opvir & Ophiactis virens & 0.2 & 2012 & \\
\hline 0.45 & 0.65 & Rock & Opvir & Ophiactis virens & 0.2 & 2012 & \\
\hline 0.45 & 0.65 & Rock & Opvir & Ophiactis virens & 0.2 & 2013 & \\
\hline 0.45 & 0.65 & Rock & Opvir & Ophiactis virens & 0.2 & 2013 & \\
\hline 0.45 & 0.65 & Rock & Opvir & Ophiactis virens & 0.2 & 2013 & \\
\hline 0.45 & 0.65 & Rock & Opvir & Ophiactis virens & 0.2 & 2013 & \\
\hline 0.45 & 0.65 & Rock & Opvir & Ophiactis virens & 0.2 & 2013 & \\
\hline 0.45 & 0.65 & Rock & Opvir & Ophiactis virens & 0.15 & 2013 & \\
\hline 0.45 & 0.65 & Rock & Opvir & Ophiactis virens & 0.15 & 2013 & \\
\hline 0.45 & 0.65 & Rock & Opvir & Ophiactis virens & 0.15 & 2013 & \\
\hline
\end{tabular}




\begin{tabular}{|c|c|c|c|c|c|c|c|}
\hline $\begin{array}{l}\text { Depth - Min } \\
\text { (m) }\end{array}$ & $\begin{array}{l}\text { Depth-Max } \\
\text { (m) }\end{array}$ & Habitats & Abr & Species & $\begin{array}{l}\text { Diameter } \\
(\mathrm{cm})\end{array}$ & Year & Month \\
\hline 0.45 & 0.65 & Rock & Opvir & Ophiactis virens & 0.15 & 2013 & \\
\hline 0.45 & 0.65 & Rock & Opvir & Ophiactis virens & 0.15 & 2013 & \\
\hline 0.45 & 0.65 & Rock & Opvir & Ophiactis virens & 0.15 & 2013 & \\
\hline 0.45 & 0.65 & Rock & Opvir & Ophiactis virens & 0.15 & 2013 & \\
\hline 0.45 & 0.65 & Rock & Opvir & Ophiactis virens & 0.15 & 2013 & \\
\hline 0.45 & 0.65 & Rock & Opvir & Ophiactis virens & 0.15 & 2013 & \\
\hline 0.45 & 0.65 & Rock & Opvir & Ophiactis virens & 0.15 & 2013 & \\
\hline 0.45 & 0.65 & Rock & Opvir & Ophiactis virens & 0.15 & 2013 & \\
\hline 0.45 & 0.65 & Rock & Opvir & Ophiactis virens & 0.15 & 2013 & \\
\hline 0.45 & 0.65 & Rock & Opvir & Ophiactis virens & 0.15 & 2013 & \\
\hline 0.45 & 0.65 & Rock & Opvir & Ophiactis virens & 0.15 & 2014 & \\
\hline 0.45 & 0.65 & Rock & Opvir & Ophiactis virens & 0.2 & 2014 & \\
\hline 0.45 & 0.65 & Rock & Opvir & Ophiactis virens & 0.2 & 2014 & \\
\hline 0.45 & 0.65 & Rock & Opvir & Ophiactis virens & 0.2 & 2014 & \\
\hline 0.45 & 0.65 & Rock & Opvir & Ophiactis virens & 0.2 & 2014 & \\
\hline 0.45 & 0.65 & Rock & Opvir & Ophiactis virens & 0.2 & 2014 & \\
\hline 0.45 & 0.65 & Rock & Opvir & Ophiactis virens & 0.2 & 2014 & \\
\hline 0.45 & 0.65 & Rock & Opvir & Ophiactis virens & 0.2 & 2014 & \\
\hline 0.45 & 0.65 & Rock & Opvir & Ophiactis virens & 0.2 & 2014 & \\
\hline 0.45 & 0.65 & Rock & Opvir & Ophiactis virens & 0.2 & 2014 & \\
\hline 0.45 & 0.65 & Rock & Opvir & Ophiactis virens & 0.2 & 2014 & \\
\hline 0.45 & 0.65 & Rock & Opvir & Ophiactis virens & 0.2 & 2014 & \\
\hline 0.45 & 0.65 & Rock & Opvir & Ophiactis virens & 0.2 & 2014 & \\
\hline 0.45 & 0.65 & Rock & Opvir & Ophiactis virens & 0.2 & 2014 & \\
\hline 0.45 & 0.65 & Rock & Opvir & Ophiactis virens & 0.2 & 2014 & \\
\hline 0.45 & 0.65 & Rock & Opvir & Ophiactis virens & 0.2 & 2014 & \\
\hline 0.45 & 0.65 & Rock & Opvir & Ophiactis virens & 0.2 & 2014 & \\
\hline 0.45 & 0.65 & Rock & Opvir & Ophiactis virens & 0.2 & 2014 & \\
\hline 0.45 & 0.65 & Rock & Opvir & Ophiactis virens & 0.2 & 2014 & \\
\hline 0.45 & 0.65 & Rock & Opvir & Ophiactis virens & 0.2 & 2014 & \\
\hline 0.45 & 0.65 & Rock & Opvir & Ophiactis virens & 0.2 & 2014 & \\
\hline 0.45 & 0.65 & Rock & Opvir & Ophiactis virens & 0.2 & 2014 & \\
\hline 0.45 & 0.65 & Rock & Opvir & Ophiactis virens & 0.2 & 2014 & \\
\hline 0.45 & 0.65 & Rock & Opvir & Ophiactis virens & 0.2 & 2014 & \\
\hline 0.45 & 0.65 & Rock & Opvir & Ophiactis virens & 0.2 & 2014 & \\
\hline 0.45 & 0.65 & Rock & Opvir & Ophiactis virens & 0.2 & 2014 & \\
\hline 0.45 & 0.65 & Rock & Opvir & Ophiactis virens & 0.2 & 2014 & \\
\hline 0.45 & 0.65 & Rock & Opvir & Ophiactis virens & 0.2 & 2014 & \\
\hline 0.45 & 0.65 & Rock & Opvir & Ophiactis virens & 0.2 & 2014 & \\
\hline 0.45 & 0.65 & Rock & Opvir & Ophiactis virens & 0.2 & 2014 & \\
\hline 0.45 & 0.65 & Rock & Opvir & Ophiactis virens & 0.25 & 2014 & \\
\hline 0.45 & 0.65 & Rock & Opvir & Ophiactis virens & 0.25 & 2014 & \\
\hline 0.45 & 0.65 & Rock & Opvir & Ophiactis virens & 0.25 & 2014 & \\
\hline 0.45 & 0.65 & Rock & Opvir & Ophiactis virens & 0.25 & 2014 & \\
\hline 0.45 & 0.65 & Rock & Opvir & Ophiactis virens & 0.25 & 2014 & \\
\hline 0.45 & 0.65 & Rock & Opvir & Ophiactis virens & 0.25 & 2014 & \\
\hline 0.45 & 0.65 & Rock & Opvir & Ophiactis virens & 0.25 & 2014 & \\
\hline 0.45 & 0.65 & Rock & Opvir & Ophiactis virens & 0.25 & 2014 & \\
\hline 0.45 & 0.65 & Rock & Opvir & Ophiactis virens & 0.25 & 2014 & \\
\hline 0.45 & 0.65 & Rock & Opvir & Ophiactis virens & 0.25 & 2014 & \\
\hline 0.45 & 0.65 & Rock & Opvir & Ophiactis virens & 0.25 & 2014 & \\
\hline 0.45 & 0.65 & Rock & Opvir & Ophiactis virens & 0.25 & 2014 & \\
\hline 0.45 & 0.65 & Rock & Opvir & Ophiactis virens & 0.25 & 2014 & \\
\hline 0.45 & 0.65 & Rock & Opvir & Ophiactis virens & 0.25 & 2014 & \\
\hline 0.45 & 0.65 & Rock & Opvir & Ophiactis virens & 0.25 & 2014 & \\
\hline 0.45 & 0.65 & Rock & Opvir & Ophiactis virens & 0.25 & 2014 & \\
\hline 0.45 & 0.65 & Rock & Opvir & Ophiactis virens & 0.25 & 2014 & \\
\hline 0.45 & 0.65 & Rock & Opvir & Ophiactis virens & 0.25 & 2014 & \\
\hline 0.45 & 0.65 & Rock & Opvir & Ophiactis virens & 0.25 & 2014 & \\
\hline 0.45 & 0.65 & Rock & Opvir & Ophiactis virens & 0.25 & 2014 & \\
\hline 0.45 & 0.65 & Rock & Opvir & Ophiactis virens & 0.25 & 2014 & \\
\hline 0.45 & 0.65 & Rock & Opvir & Ophiactis virens & 0.25 & 2014 & \\
\hline 0.45 & 0.65 & Rock & Opvir & Ophiactis virens & 0.25 & 2014 & \\
\hline 0.45 & 0.65 & Rock & Opvir & Ophiactis virens & 0.25 & 2014 & \\
\hline 0.45 & 0.65 & Rock & Opvir & Ophiactis virens & 0.25 & 2014 & \\
\hline 0.45 & 0.65 & Rock & Opvir & Ophiactis virens & 0.25 & 2014 & \\
\hline 0.45 & 0.65 & Rock & Opvir & Ophiactis virens & 0.25 & 2014 & \\
\hline 0.45 & 0.65 & Rock & Opvir & Ophiactis virens & 0.25 & 2014 & \\
\hline 0.45 & 0.65 & Rock & Opvir & Ophiactis virens & 0.25 & 2014 & \\
\hline 0.45 & 0.65 & Rock & Opvir & Ophiactis virens & 0.25 & 2014 & \\
\hline 0.45 & 0.65 & Rock & Opvir & Ophiactis virens & 0.25 & 2014 & \\
\hline 0.45 & 0.65 & Rock & Opvir & Ophiactis virens & 0.25 & 2014 & \\
\hline 0.45 & 0.65 & Rock & Opvir & Ophiactis virens & 0.25 & 2014 & \\
\hline 0.45 & 0.65 & Rock & Opvir & Ophiactis virens & 0.25 & 2014 & \\
\hline 0.45 & 0.65 & Rock & Opvir & Ophiactis virens & 0.25 & 2014 & \\
\hline 0.45 & 0.65 & Rock & Opvir & Ophiactis virens & 0.25 & 2014 & \\
\hline 0.45 & 0.65 & Rock & Opvir & Ophiactis virens & 0.25 & 2014 & \\
\hline
\end{tabular}


S18 • H. Chammem et al.

\begin{tabular}{|c|c|c|c|c|c|c|c|}
\hline $\begin{array}{l}\text { Depth - Min } \\
(\mathrm{m})\end{array}$ & $\begin{array}{c}\text { Depth-Max } \\
(\mathrm{m})\end{array}$ & Habitats & Abr & Species & $\begin{array}{l}\text { Diameter } \\
(\mathrm{cm})\end{array}$ & Year & Month \\
\hline 0.45 & 0.65 & Rock & Opvir & Ophiactis virens & 0.25 & 2014 & \\
\hline 0.45 & 0.65 & Rock & Opvir & Ophiactis virens & 0.25 & 2014 & \\
\hline 0.45 & 0.65 & Rock & Opvir & Ophiactis virens & 0.25 & 2014 & \\
\hline 0.45 & 0.65 & Rock & Opvir & Ophiactis virens & 0.25 & 2014 & \\
\hline 0.45 & 0.65 & Rock & Opvir & Ophiactis virens & 0.25 & 2014 & \\
\hline 0.45 & 0.65 & Rock & Opvir & Ophiactis virens & 0.25 & 2014 & \\
\hline 0.45 & 0.65 & Rock & Opvir & Ophiactis virens & 0.25 & 2014 & \\
\hline 0.45 & 0.65 & Rock & Opvir & Ophiactis virens & 0.25 & 2014 & \\
\hline 0.45 & 0.65 & Rock & Opvir & Ophiactis virens & 0.25 & 2014 & \\
\hline 0.45 & 0.65 & Rock & Opvir & Ophiactis virens & 0.25 & 2014 & \\
\hline 0.45 & 0.65 & Rock & Opvir & Ophiactis virens & 0.25 & 2014 & \\
\hline 0.45 & 0.65 & Rock & Opvir & Ophiactis virens & 0.25 & 2014 & \\
\hline 0.45 & 0.65 & Rock & Opvir & Ophiactis virens & 0.25 & 2014 & \\
\hline 0.45 & 0.65 & Rock & Opvir & Ophiactis virens & 0.25 & 2014 & \\
\hline 0.45 & 0.65 & Rock & Opvir & Ophiactis virens & 0.25 & 2014 & \\
\hline 0.45 & 0.65 & Rock & Opvir & Ophiactis virens & 0.3 & 2014 & \\
\hline 0.45 & 0.65 & Rock & Opvir & Ophiactis virens & 0.3 & 2014 & \\
\hline 0.45 & 0.65 & Rock & Opvir & Ophiactis virens & 0.3 & 2014 & \\
\hline 0.45 & 0.65 & Rock & Opvir & Ophiactis virens & 0.3 & 2014 & \\
\hline 0.45 & 0.65 & Rock & Opvir & Ophiactis virens & 0.3 & 2014 & \\
\hline 0.45 & 0.65 & Rock & Opvir & Ophiactis virens & 0.3 & 2014 & \\
\hline 0.45 & 0.65 & Rock & Opvir & Ophiactis virens & 0.3 & 2014 & \\
\hline 0.45 & 0.65 & Rock & Opvir & Ophiactis virens & 0.3 & 2014 & \\
\hline 0.45 & 0.65 & Rock & Opvir & Ophiactis virens & 0.3 & 2014 & \\
\hline 0.45 & 0.65 & Rock & Opvir & Ophiactis virens & 0.3 & 2014 & \\
\hline 0.45 & 0.65 & Rock & Opvir & Ophiactis virens & 0.3 & 2014 & \\
\hline 0.45 & 0.65 & Rock & Opvir & Ophiactis virens & 0.3 & 2014 & \\
\hline 0.45 & 0.65 & Rock & Opvir & Ophiactis virens & 0.3 & 2014 & \\
\hline 0.45 & 0.65 & Rock & Opvir & Ophiactis virens & 0.3 & 2014 & \\
\hline 0.45 & 0.65 & Rock & Opvir & Ophiactis virens & 0.3 & 2014 & \\
\hline 0.45 & 0.65 & Rock & Opvir & Ophiactis virens & 0.3 & 2014 & \\
\hline 0.45 & 0.65 & Rock & Opvir & Ophiactis virens & 0.3 & 2014 & \\
\hline 0.45 & 0.65 & Rock & Opvir & Ophiactis virens & 0.3 & 2014 & \\
\hline 0.45 & 0.65 & Rock & Opvir & Ophiactis virens & 0.3 & 2014 & \\
\hline 0.45 & 0.65 & Rock & Opvir & Ophiactis virens & 0.3 & 2014 & \\
\hline 0.45 & 0.65 & Rock & Opvir & Ophiactis virens & 0.3 & 2014 & \\
\hline 0.45 & 0.65 & Rock & Opvir & Ophiactis virens & 0.3 & 2014 & \\
\hline 0.45 & 0.65 & Rock & Opvir & Ophiactis virens & 0.3 & 2014 & \\
\hline 0.45 & 0.65 & Rock & Opvir & Ophiactis virens & 0.3 & 2014 & \\
\hline 0.45 & 0.65 & Rock & Opvir & Ophiactis virens & 0.3 & 2014 & \\
\hline 0.45 & 0.65 & Rock & Opvir & Ophiactis virens & 0.3 & 2014 & \\
\hline 0.45 & 0.65 & Rock & Opvir & Ophiactis virens & 0.3 & 2014 & \\
\hline 0.45 & 0.65 & Rock & Opvir & Ophiactis virens & 0.3 & 2014 & \\
\hline 0.45 & 0.65 & Rock & Opvir & Ophiactis virens & 0.3 & 2014 & \\
\hline 0.45 & 0.65 & Algea & Opvir & Ophiactis virens & 0.3 & 2014 & \\
\hline 0.45 & 0.65 & Algea & Opvir & Ophiactis virens & 0.3 & 2014 & \\
\hline 0.45 & 0.65 & Algea & Opvir & Ophiactis virens & 0.3 & 2014 & \\
\hline 0.45 & 0.65 & Algea & Opvir & Ophiactis virens & 0.3 & 2014 & \\
\hline 0.45 & 0.65 & Algea & Opvir & Ophiactis virens & 0.3 & 2014 & \\
\hline 0.45 & 0.65 & Algea & Opvir & Ophiactis virens & 0.3 & 2014 & \\
\hline 0.45 & 0.65 & Algea & Opvir & Ophiactis virens & 0.3 & 2014 & \\
\hline 0.45 & 0.65 & Algea & Opvir & Ophiactis virens & 0.3 & 2014 & \\
\hline 0.45 & 0.65 & Algea & Opvir & Ophiactis virens & 0.3 & 2014 & \\
\hline 0.45 & 0.65 & Algea & Opvir & Ophiactis virens & 0.3 & 2014 & \\
\hline 0.45 & 0.65 & Algea & Opvir & Ophiactis virens & 0.3 & 2014 & \\
\hline 0.45 & 0.65 & Algea & Opvir & Ophiactis virens & 0.3 & 2014 & \\
\hline 0.45 & 0.65 & Algea & Opvir & Ophiactis virens & 0.3 & 2014 & \\
\hline 0.45 & 0.65 & Algea & Opvir & Ophiactis virens & 0.3 & 2014 & \\
\hline 0.45 & 0.65 & Algea & Opvir & Ophiactis virens & 0.3 & 2014 & \\
\hline 0.45 & 0.65 & Algea & Opvir & Ophiactis virens & 0.3 & 2014 & \\
\hline 0.45 & 0.65 & Algea & Opvir & Ophiactis virens & 0.3 & 2014 & \\
\hline 0.45 & 0.65 & Algea & Opvir & Ophiactis virens & 0.3 & 2014 & \\
\hline 0.45 & 0.65 & Algea & Opvir & Ophiactis virens & 0.3 & 2014 & \\
\hline 0.45 & 0.65 & Algea & Opvir & Ophiactis virens & 0.3 & 2014 & \\
\hline 0.45 & 0.65 & Algea & Opvir & Ophiactis virens & 0.3 & 2014 & \\
\hline 0.45 & 0.65 & Algea & Opvir & Ophiactis virens & 0.3 & 2014 & \\
\hline 0.45 & 0.65 & Algea & Opvir & Ophiactis virens & 0.3 & 2014 & \\
\hline 0.45 & 0.65 & Algea & Opvir & Ophiactis virens & 0.3 & 2014 & \\
\hline 0.45 & 0.65 & Algea & Opvir & Ophiactis virens & 0.3 & 2014 & \\
\hline 0.45 & 0.65 & Algea & Opvir & Ophiactis virens & 0.3 & 2014 & \\
\hline 0.45 & 0.65 & Algea & Opvir & Ophiactis virens & 0.3 & 2014 & \\
\hline 0.45 & 0.65 & Algea & Opvir & Ophiactis virens & 0.3 & 2016 & \\
\hline 0.45 & 0.65 & Algea & Opvir & Ophiactis virens & 0.3 & 2016 & \\
\hline 0.45 & 0.65 & Algea & Opvir & Ophiactis virens & 0.3 & 2016 & \\
\hline 0.45 & 0.65 & Algea & Opvir & Ophiactis virens & 0.3 & 2016 & \\
\hline 0.45 & 0.65 & Algea & Opvir & Ophiactis virens & 0.3 & 2016 & \\
\hline 0.45 & 0.65 & Algea & Opvir & Ophiactis virens & 0.3 & 2016 & \\
\hline
\end{tabular}




\begin{tabular}{|c|c|c|c|c|c|c|c|}
\hline $\begin{array}{l}\text { Depth - Min } \\
\text { (m) }\end{array}$ & $\begin{array}{l}\text { Depth-Max } \\
\text { (m) }\end{array}$ & Habitats & Abr & Species & $\begin{array}{l}\text { Diameter } \\
(\mathrm{cm})\end{array}$ & Year & Month \\
\hline 0.45 & 0.65 & Algea & Opvir & Ophiactis virens & 0.3 & 2016 & \\
\hline 51 & 58 & Mud & Opnig & Ophiocomina nigra & 5 & 2016 & June \\
\hline 0.2 & 0.6 & Algea & Oplon & Ophioderma longicauda & 3 & 2012 & December \\
\hline 98 & 105 & Sand & Oppen & Ophiomyxa pentagona & 2 & 2015 & October \\
\hline 98 & 105 & Sand & Oppen & Ophiomyxa pentagona & 2 & 2015 & October \\
\hline 98 & 105 & Sand & Oppen & Ophiomyxa pentagona & 2 & 2015 & October \\
\hline 91 & 95 & Sand & Oppen & Ophiomyxa pentagona & 2 & 2015 & October \\
\hline 91 & 95 & Sand & Oppen & Ophiomyxa pentagona & 2 & 2015 & October \\
\hline 91 & 95 & Sand & Oppen & Ophiomyxa pentagona & 2 & 2015 & October \\
\hline 91 & 95 & Sand & Oppen & Ophiomyxa pentagona & 4 & 2015 & October \\
\hline 91 & 95 & Sand & Oppen & Ophiomyxa pentagona & 4 & 2015 & October \\
\hline 166 & 199 & Sand & Oppen & Ophiomyxa pentagona & 4 & 2015 & October \\
\hline 166 & 199 & Sand & Oppen & Ophiomyxa pentagona & 4 & 2015 & October \\
\hline 166 & 199 & Sand & Oppen & Ophiomyxa pentagona & 4 & 2015 & October \\
\hline 166 & 199 & Sand & Oppen & Ophiomyxa pentagona & 4 & 2015 & October \\
\hline 180 & 210 & Sand & Oppen & Ophiomyxa pentagona & 4 & 2015 & October \\
\hline 180 & 210 & Sand & Oppen & Ophiomyxa pentagona & 4 & 2015 & October \\
\hline 180 & 210 & Sand & Oppen & Ophiomyxa pentagona & 4 & 2015 & October \\
\hline 120 & 145 & Mud & Oppen & Ophiomyxa pentagona & 4 & 2015 & October \\
\hline 120 & 145 & Mud & Oppen & Ophiomyxa pentagona & 4 & 2015 & October \\
\hline 120 & 145 & Mud & Oppen & Ophiomyxa pentagona & 4 & 2015 & December \\
\hline 67 & 70 & Sand & Oppen & Ophiomvxa pentagona & 4 & 2015 & December \\
\hline 67 & 70 & Sand & Oppen & Ophiomyxa pentagona & 4 & 2015 & December \\
\hline 67 & 70 & Sand & Oppen & Ophiomyxa pentagona & 4 & 2015 & December \\
\hline 67 & 70 & Sand & Oppen & Ophiomyxa pentagona & 4 & 2015 & December \\
\hline 90 & 105 & Mud-Sand & Oppen & Ophiomyxa pentagona & 4 & 2015 & December \\
\hline 90 & 105 & Mud-Sand & Oppen & Ophiomyxa pentagona & 4 & 2015 & December \\
\hline 90 & 105 & Mud-Sand & Oppen & Ophiomyxa pentagona & 4 & 2016 & March \\
\hline 90 & 110 & Mud-Sand & Oppen & Ophiomyxa pentagona & 4 & 2016 & March \\
\hline 90 & 110 & Mud-Sand & Oppen & Ophiomyxa pentagona & 4 & 2016 & March \\
\hline 80 & 120 & Sand & Oppen & Ophiomyxa pentagona & 4 & 2016 & March \\
\hline 80 & 120 & Sand & Oppen & Ophiomyxa pentagona & 4 & 2016 & March \\
\hline 79 & 80 & Sand & Oppen & Ophiomyxa pentagona & 4 & 2016 & March \\
\hline 79 & 80 & Sand & Oppen & Ophiomyxa pentagona & 4 & 2016 & March \\
\hline 79 & 80 & Sand & Oppen & Ophiomyxa pentagona & 4 & 2016 & March \\
\hline 79 & 80 & Sand & Oppen & Ophiomyxa pentagona & 4 & 2016 & March \\
\hline 79 & 80 & Sand & Oppen & Ophiomyxa pentagona & 4 & 2016 & March \\
\hline 79 & 80 & Sand & Oppen & Ophiomyxa pentagona & 4 & 2016 & March \\
\hline 90 & 120 & Sand & Oppen & Ophiomyxa pentagona & 4 & 2016 & March \\
\hline 90 & 120 & Sand & Oppen & Ophiomyxa pentagona & 4 & 2016 & March \\
\hline 90 & 120 & Sand & Oppen & Ophiomyxa pentagona & 4 & 2016 & March \\
\hline 100 & 115 & Sand & Oppen & Ophiomyxa pentagona & 4 & 2016 & August \\
\hline 100 & 115 & Sand & Oppen & Ophiomyxa pentagona & 4 & 2016 & August \\
\hline 100 & 115 & Sand & Oppen & Ophiomyxa pentagona & 4 & 2016 & August \\
\hline 100 & 115 & Sand & Oppen & Ophiomyxa pentagona & 4 & 2016 & August \\
\hline 100 & 125 & Sand & Oppen & Ophiomyxa pentagona & 4 & 2016 & August \\
\hline 100 & 125 & Sand & Oppen & Ophiomyxa pentagona & 4 & 2016 & August \\
\hline 100 & 125 & Sand & Oppen & Ophiomyxa pentagona & 4 & 2016 & August \\
\hline 100 & 125 & Sand & Oppen & Ophiomyxa pentagona & 4.5 & 2016 & August \\
\hline 50 & 60 & Rock & Oppen & Ophiomyxa pentagona & 4.5 & 2016 & August \\
\hline 50 & 60 & Rock & Oppen & Ophiomyxa pentagona & 4.5 & 2016 & August \\
\hline 50 & 60 & Rock & Oppen & Ophiomyxa pentagona & 4.5 & 2016 & August \\
\hline 50 & 60 & Rock & Oppen & Ophiomyxa pentagona & 4.5 & 2016 & August \\
\hline 85 & 100 & Sand & Oppen & Ophiomyxa pentagona & 4.5 & 2016 & April \\
\hline 85 & 100 & Sand & Oppen & Ophiomyxa pentagona & 4.5 & 2016 & April \\
\hline 85 & 100 & Sand & Oppen & Ophiomyxa pentagona & 4.5 & 2016 & April \\
\hline 85 & 100 & Sand & Oppen & Ophiomyxa pentagona & 4.5 & 2016 & April \\
\hline 152 & 177 & Sand & Oppen & Ophiomyxa pentagona & 4.5 & 2016 & April \\
\hline 152 & 177 & Sand & Oppen & Ophiomyxa pentagona & 4.5 & 2016 & April \\
\hline 152 & 177 & Sand & Oppen & Ophiomyxa pentagona & 4.5 & 2016 & April \\
\hline 152 & 177 & Sand & Oppen & Ophiomyxa pentagona & 4.5 & 2016 & April \\
\hline 152 & 177 & Sand & Oppen & Ophiomyxa pentagona & 4.5 & 2016 & April \\
\hline 152 & 177 & Sand & Oppen & Ophiomyxa pentagona & 4.5 & 2016 & April \\
\hline 78 & 100 & Sand & Oppen & Ophiomyxa pentagona & 4.5 & 2016 & June \\
\hline 78 & 100 & Sand & Oppen & Ophiomyxa pentagona & 4.5 & 2016 & June \\
\hline 78 & 100 & Sand & Oppen & Ophiomyxa pentagona & 4.5 & 2016 & June \\
\hline 78 & 100 & Sand & Oppen & Ophiomyxa pentagona & 4.5 & 2016 & June \\
\hline 78 & 100 & Sand & Oppen & Ophiomyxa pentagona & 4.5 & 2016 & June \\
\hline 78 & 100 & Sand & Oppen & Ophiomyxa pentagona & 5 & 2016 & June \\
\hline 78 & 100 & Sand & Oppen & Ophiomyxa pentagona & 5 & 2016 & June \\
\hline 78 & 100 & Sand & Oppen & Ophiomyxa pentagona & 5 & 2016 & June \\
\hline 78 & 100 & Sand & Oppen & Ophiomyxa pentagona & 5 & 2016 & June \\
\hline 51 & 58 & Sand & Oppen & Ophiomyxa pentagona & 5 & 2016 & June \\
\hline 51 & 58 & Sand & Oppen & Ophiomyxa pentagona & 5 & 2016 & June \\
\hline 51 & 58 & Sand & Oppen & Ophiomyxa pentagona & 5 & 2016 & June \\
\hline 51 & 58 & Sand & Oppen & Ophiomyxa pentagona & 5 & 2016 & September \\
\hline 130 & 140 & Sand & Oppen & Ophiomyxa pentagona & 5 & 2016 & September \\
\hline
\end{tabular}


S20 • H. Chammem et al.

\begin{tabular}{|c|c|c|c|c|c|c|c|}
\hline $\begin{array}{l}\text { Depth - Min } \\
(\mathrm{m})\end{array}$ & $\begin{array}{c}\text { Depth-Max } \\
(\mathrm{m})\end{array}$ & Habitats & Abr & Species & $\begin{array}{l}\text { Diameter } \\
(\mathrm{cm})\end{array}$ & Year & Month \\
\hline 130 & 140 & Sand & Oppen & Ophiomyxa pentagona & 5 & 2016 & September \\
\hline 130 & 140 & Sand & Oppen & Ophiomyxa pentagona & 5 & 2016 & September \\
\hline 130 & 140 & Sand & Oppen & Ophiomyxa pentagona & 5 & 2016 & September \\
\hline 70 & 95 & Sand & Oppen & Ophiomyxa pentagona & 5 & 2016 & September \\
\hline 70 & 95 & Sand & Oppen & Ophiomyxa pentagona & 5 & 2016 & September \\
\hline 70 & 95 & Sand & Oppen & Ophiomyxa pentagona & 5 & 2016 & September \\
\hline 70 & 95 & Sand & Oppen & Ophiomyxa pentagona & 5 & 2016 & September \\
\hline 81 & 100 & Sand & Opqui & Ophiothrix quinquemaculata & 1 & 2016 & June \\
\hline 81 & 100 & Sand & Opqui & Ophiothrix quinquemaculata & 1 & 2016 & June \\
\hline 81 & 100 & Sand & Opqui & Ophiothrix quinquemaculata & 1 & 2016 & June \\
\hline 81 & 100 & Sand & Opqui & Ophiothrix quinquemaculata & 1 & 2016 & June \\
\hline 81 & 100 & Sand & Opqui & Ophiothrix quinquemaculata & 1 & 2016 & June \\
\hline 81 & 100 & Sand & Opqui & Ophiothrix quinquemaculata & 1 & 2016 & June \\
\hline 90 & 160 & Mud-Sand & Opqui & Ophiothrix quinquemaculata & 1 & 2016 & June \\
\hline 90 & 160 & Mud-Sand & Opqui & Ophiothrix quinquemaculata & 1.5 & 2016 & June \\
\hline 90 & 160 & Mud-Sand & Opqui & Ophiothrix quinquemaculata & 1.5 & 2016 & June \\
\hline 90 & 160 & Mud-Sand & Opqui & Ophiothrix quinquemaculata & 1.5 & 2016 & June \\
\hline 72 & 90 & Rock & Opqui & Ophiothrix quinquemaculata & 1.5 & 2016 & June \\
\hline 72 & 90 & Rock & Opqui & Ophiothrix quinquemaculata & 1.5 & 2016 & June \\
\hline 72 & 90 & Rock & Opqui & Ophiothrix quinquemaculata & 2 & 2016 & June \\
\hline 72 & 90 & Rock & Opqui & Ophiothrix quinquemaculata & 2 & 2016 & June \\
\hline 78 & 84 & Rock & Opqui & Ophiothrix quinquemaculata & 2 & 2016 & June \\
\hline 78 & 84 & Rock & Opqui & Ophiothrix quinquemaculata & 2 & 2016 & June \\
\hline 78 & 84 & Rock & Opqui & Ophiothrix quinquemaculata & 4 & 2016 & June \\
\hline 78 & 84 & Rock & Opqui & Ophiothrix quinquemaculata & 4 & 2016 & June \\
\hline 135 & 170 & Sand & Opqui & Ophiothrix quinquemaculata & 4 & 2016 & June \\
\hline 135 & 170 & Sand & Opqui & Ophiothrix quinquemaculata & 4 & 2016 & June \\
\hline 135 & 170 & Sand & Opqui & Ophiothrix quinquemaculata & 4 & 2016 & June \\
\hline 3 & 5 & Sand & Opoph & Ophiura ophiura & 3 & 2012 & \\
\hline 3 & 5 & Sand & Opoph & Ophiura ophiura & 3 & 2012 & \\
\hline 3 & 5 & Sand & Opoph & Ophiura ophiura & 3 & 2012 & \\
\hline 3 & 5 & Sand & Opoph & Ophiura ophiura & 3 & 2012 & \\
\hline 3 & 5 & Sand & Opoph & Ophiura ophiura & 3 & 2012 & \\
\hline 3 & 5 & Sand & Opoph & Ophiura ophiura & 3 & 2012 & \\
\hline 3 & 5 & Sand & Opoph & Ophiura ophiura & 3 & 2012 & \\
\hline 3 & 5 & Sand & Opoph & Ophiura ophiura & 3 & 2012 & \\
\hline 3 & 5 & Sand & Opoph & Ophiura ophiura & 3 & 2012 & \\
\hline 3 & 5 & Sand & Opoph & Ophiura ophiura & 3 & 2012 & \\
\hline 3 & 5 & Sand & Opoph & Ophiura ophiura & 3 & 2012 & \\
\hline 3 & 5 & Sand & Opoph & Ophiura ophiura & 3 & 2012 & \\
\hline 3 & 5 & Sand & Opoph & Ophiura ophiura & 3 & 2012 & \\
\hline 3 & 5 & Sand & Opoph & Ophiura ophiura & 3 & 2012 & \\
\hline 3 & 5 & Sand & Opoph & Ophiura ophiura & 3 & 2012 & \\
\hline 3 & 5 & Sand & Opoph & Ophiura ophiura & 3 & 2012 & \\
\hline 3 & 5 & Sand & Opoph & Ophiura ophiura & 3 & 2012 & \\
\hline 3 & 5 & Sand & Opoph & Ophiura ophiura & 3 & 2012 & \\
\hline 91 & 95 & Sand & Opoph & Ophiura ophiura & 4 & 2015 & October \\
\hline 91 & 95 & Sand & Opoph & Ophiura ophiura & 4 & 2015 & October \\
\hline 91 & 95 & Sand & Opoph & Ophiura ophiura & 4 & 2015 & October \\
\hline 91 & 95 & Sand & Opoph & Ophiura ophiura & 4 & 2015 & October \\
\hline 68 & 94 & Mud-Sand & Opoph & Ophiura ophiura & 4 & 2015 & October \\
\hline 68 & 94 & Mud-Sand & Opoph & Ophiura ophiura & 4 & 2015 & October \\
\hline 68 & 94 & Mud-Sand & Opoph & Ophiura ophiura & 4 & 2015 & October \\
\hline 91 & 95 & Sand & Opoph & Ophiura ophiura & 4 & 2015 & December \\
\hline 91 & 95 & Sand & Opoph & Ophiura ophiura & 4 & 2015 & December \\
\hline 91 & 95 & Sand & Opoph & Ophiura ophiura & 4 & 2015 & December \\
\hline 91 & 95 & Sand & Opoph & Ophiura ophiura & 4 & 2015 & December \\
\hline 67 & 70 & Sand & Opoph & Ophiura ophiura & 4 & 2015 & December \\
\hline 67 & 70 & Sand & Opoph & Ophiura ophiura & 4 & 2015 & December \\
\hline 67 & 70 & Sand & Opoph & Ophiura ophiura & 4 & 2015 & December \\
\hline 80 & 111 & Mud & Opoph & Ophiura ophiura & 4 & 2015 & December \\
\hline 80 & 111 & Mud & Opoph & Ophiura ophiura & 4 & 2015 & December \\
\hline 80 & 111 & Mud & Opoph & Ophiura ophiura & 4 & 2016 & March \\
\hline 80 & 111 & Mud & Opoph & Ophiura ophiura & 4 & 2016 & March \\
\hline 68 & 77 & Sand & Opoph & Ophiura ophiura & 4 & 2016 & March \\
\hline 68 & 77 & Sand & Opoph & Ophiura ophiura & 4 & 2016 & March \\
\hline 68 & 77 & Sand & Opoph & Ophiura ophiura & 4 & 2016 & March \\
\hline 68 & 77 & Sand & Opoph & Ophiura ophiura & 4 & 2016 & March \\
\hline 90 & 110 & Mud-Sand & Opoph & Ophiura ophiura & 4 & 2016 & March \\
\hline 90 & 110 & Mud-Sand & Opoph & Ophiura ophiura & 4 & 2016 & March \\
\hline 90 & 110 & Mud-Sand & Opoph & Ophiura ophiura & 4 & 2016 & March \\
\hline 90 & 110 & Mud-Sand & Opoph & Ophiura ophiura & 4 & 2016 & March \\
\hline 90 & 110 & Mud-Sand & Opoph & Ophiura ophiura & 4 & 2016 & March \\
\hline 79 & 80 & Sand & Opoph & Ophiura ophiura & 4 & 2016 & March \\
\hline 79 & 80 & Sand & Opoph & Ophiura ophiura & 4 & 2016 & March \\
\hline 79 & 80 & Sand & Opoph & Ophiura ophiura & 4 & 2016 & March \\
\hline 79 & 80 & Sand & Opoph & Ophiura ophiura & 4 & 2016 & March \\
\hline
\end{tabular}




\begin{tabular}{|c|c|c|c|c|c|c|c|}
\hline $\begin{array}{l}\text { Depth - Min } \\
(\mathrm{m})\end{array}$ & $\begin{array}{c}\text { Depth-Max } \\
(\mathrm{m})\end{array}$ & Habitats & Abr & Species & $\begin{array}{l}\text { Diameter } \\
(\mathrm{cm})\end{array}$ & Year & Month \\
\hline 79 & 80 & Sand & Opoph & Ophiura ophiura & 4 & 2016 & March \\
\hline 80 & 113 & Mud & Opoph & Ophiura ophiura & 4 & 2016 & August \\
\hline 80 & 113 & Mud & Opoph & Ophiura ophiura & 4 & 2016 & August \\
\hline 80 & 113 & Mud & Opoph & Ophiura ophiura & 4 & 2016 & August \\
\hline 80 & 113 & Mud & Opoph & Ophiura ophiura & 5 & 2016 & August \\
\hline 70 & 100 & Sand & Opoph & Ophiura ophiura & 5 & 2016 & August \\
\hline 70 & 100 & Sand & Opoph & Ophiura ophiura & 5 & 2016 & August \\
\hline 70 & 100 & Sand & Opoph & Ophiura ophiura & 5 & 2016 & August \\
\hline 70 & 100 & Sand & Opoph & Ophiura ophiura & 5 & 2016 & August \\
\hline 75 & 101 & Rock & Opoph & Ophiura ophiura & 5 & 2016 & August \\
\hline 75 & 101 & Rock & Opoph & Ophiura ophiura & 5 & 2016 & August \\
\hline 75 & 101 & Rock & Opoph & Ophiura ophiura & 5 & 2016 & August \\
\hline 75 & 101 & Rock & Opoph & Ophiura ophiura & 5 & 2016 & August \\
\hline 75 & 101 & Rock & Opoph & Ophiura ophiura & 5 & 2016 & August \\
\hline 50 & 103 & Sand & Opoph & Ophiura ophiura & 5 & 2016 & August \\
\hline 50 & 103 & Sand & Opoph & Ophiura ophiura & 5 & 2016 & August \\
\hline 50 & 103 & Sand & Opoph & Ophiura ophiura & 5 & 2016 & August \\
\hline 152 & 177 & Sand & Opoph & Ophiura ophiura & 5 & 2016 & April \\
\hline 152 & 177 & Sand & Opoph & Ophiura ophiura & 5 & 2016 & April \\
\hline 152 & 177 & Sand & Opoph & Ophiura ophiura & 5 & 2016 & April \\
\hline 152 & 177 & Sand & Opoph & Ophiura ophiura & 5 & 2016 & April \\
\hline 152 & 177 & Sand & Opoph & Ophiura ophiura & 5 & 2016 & April \\
\hline 152 & 177 & Sand & Opoph & Ophiura ophiura & 5 & 2016 & April \\
\hline 152 & 177 & Sand & Opoph & Ophiura ophiura & 5 & 2016 & April \\
\hline 152 & 177 & Sand & Opoph & Ophiura ophiura & 5 & 2016 & June \\
\hline 152 & 100 & Mud-Sand & Opoph & Ophiura ophiura & 5 & 2016 & June \\
\hline 78 & 100 & Mud-Sand & Opoph & Ophiura ophiura & 5 & 2016 & June \\
\hline 78 & 100 & Mud-Sand & Opoph & Ophiura ophiura & 5 & 2016 & June \\
\hline 78 & 100 & Mud-Sand & Opoph & Ophiura ophiura & 5 & 2016 & June \\
\hline 51 & 58 & Sand & Opoph & Ophiura ophiura & 5 & 2016 & June \\
\hline 51 & 58 & Sand & Opoph & Ophiura ophiura & 5 & 2016 & June \\
\hline 51 & 58 & Sand & Opoph & Ophiura ophiura & 5 & 2016 & June \\
\hline 51 & 58 & Sand & Opoph & Ophiura ophiura & 5 & 2016 & June \\
\hline 130 & 140 & Sand & Opoph & Ophiura ophiura & 5 & 2016 & June \\
\hline 130 & 140 & Sand & Opoph & Ophiura ophiura & 5 & 2016 & June \\
\hline 130 & 140 & Sand & Opoph & Ophiura ophiura & 5 & 2016 & September \\
\hline 130 & 140 & Sand & Opoph & Ophiura ophiura & 5 & 2016 & September \\
\hline 72 & 90 & Rock & Opoph & Ophiura ophiura & 5 & 2016 & September \\
\hline 72 & 90 & Rock & Opoph & Ophiura ophiura & 5 & 2016 & September \\
\hline 72 & 90 & Rock & Opoph & Ophiura ophiura & 5 & 2016 & September \\
\hline 72 & 90 & Rock & Opoph & Ophiura ophiura & 5 & 2016 & September \\
\hline 72 & 90 & Rock & Opoph & Ophiura ophiura & 5 & 2016 & September \\
\hline 72 & 90 & Rock & Opoph & Ophiura ophiura & 5 & 2016 & September \\
\hline 135 & 170 & Sand & Opoph & Ophiura ophiura & 5 & 2016 & September \\
\hline 135 & 170 & Sand & Opoph & Ophiura ophiura & 5 & 2016 & September \\
\hline 135 & 170 & Sand & Opoph & Ophiura ophiura & 5 & 2016 & September \\
\hline 160 & 194 & Mud & Opoph & Ophiura ophiura & 5 & 2016 & July \\
\hline 160 & 194 & Mud & Opoph & Ophiura ophiura & 5 & 2016 & July \\
\hline 160 & 194 & Mud & Opoph & Ophiura ophiura & 5 & 2016 & July \\
\hline 180 & 192 & Mud & Opoph & Ophiura ophiura & 5 & 2016 & July \\
\hline 180 & 192 & Mud & Opoph & Ophiura ophiura & 5 & 2016 & July \\
\hline 180 & 192 & Mud & Opoph & Ophiura ophiura & 5 & 2016 & July \\
\hline 175 & 193 & Sand & Opoph & Ophiura ophiura & 5 & 2016 & July \\
\hline 175 & 193 & Sand & Opoph & Ophiura ophiura & 5 & 2016 & July \\
\hline 175 & 193 & Sand & Opoph & Ophiura ophiura & 5 & 2016 & July \\
\hline 176 & 179 & Mud-Sand & Opoph & Ophiura ophiura & 5 & 2016 & July \\
\hline 176 & 179 & Mud-Sand & Opoph & Ophiura ophiura & 5 & 2016 & July \\
\hline 176 & 179 & Mud-Sand & Opoph & Ophiura ophiura & 5 & 2016 & July \\
\hline 183 & 193 & Sand & Opoph & Ophiura ophiura & 5 & 2016 & July \\
\hline 183 & 193 & Sand & Opoph & Ophiura ophiura & 5 & 2016 & July \\
\hline 142 & 163 & Mud & Opoph & Ophiura ophiura & 5 & 2016 & July \\
\hline 142 & 163 & Mud & Opoph & Ophiura ophiura & 5 & 2016 & July \\
\hline 142 & 163 & Mud & Opoph & Ophiura ophiura & 5 & 2016 & July \\
\hline 181 & 186 & Mud-Sand & Opoph & Ophiura ophiura & 5 & 2016 & July \\
\hline 181 & 186 & Mud-Sand & Opoph & Ophiura ophiura & 5 & 2016 & July \\
\hline 181 & 186 & Mud-Sand & Opoph & Ophiura ophiura & 5 & 2016 & July \\
\hline 181 & 186 & Mud-Sand & Opoph & Ophiura ophiura & 5 & 2016 & July \\
\hline 179 & 185 & Sand & Opoph & Ophiura ophiura & 5 & 2016 & July \\
\hline 179 & 185 & Sand & Opoph & Ophiura ophiura & 5 & 2016 & July \\
\hline 179 & 185 & Sand & Opoph & Ophiura ophiura & 5 & 2016 & July \\
\hline 181 & 190 & Sand & Opoph & Ophiura ophiura & 5 & 2016 & July \\
\hline 181 & 190 & Sand & Opoph & Ophiura ophiura & 5 & 2016 & July \\
\hline 181 & 190 & Sand & Opoph & Ophiura ophiura & 5 & 2016 & July \\
\hline 181 & 190 & Sand & Opoph & Ophiura ophiura & 5 & 2016 & July \\
\hline 181 & 190 & Sand & Opoph & Ophiura ophiura & 5 & 2016 & July \\
\hline 3 & 5 & Sand & Opsav & Ophiactis savignyi & 0.5 & 2016 & \\
\hline
\end{tabular}

